\title{
General and Mild Cobalt-Catalyzed C-Alkylation of Unactivated Amides and Esters with Alcohols
}

\author{
Nicklas Deibl and Rhett Kempe* \\ kempe@uni-bayreuth.de \\ Anorganische Chemie II-Katalysatordesign, Universität Bayreuth, 95440 Bayreuth, Germany
}

\section{Table of contents}

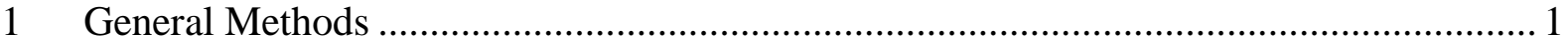

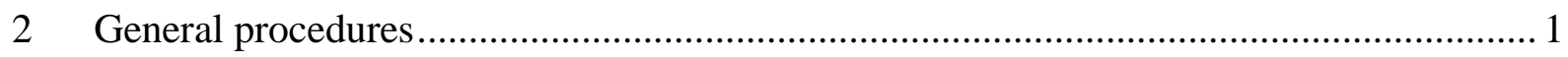

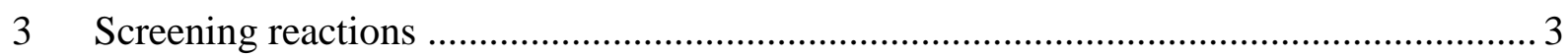

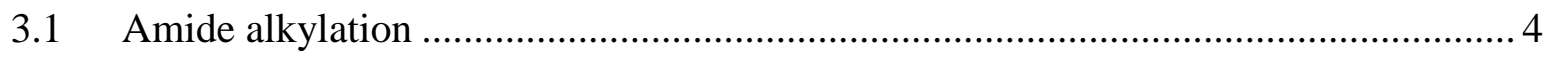

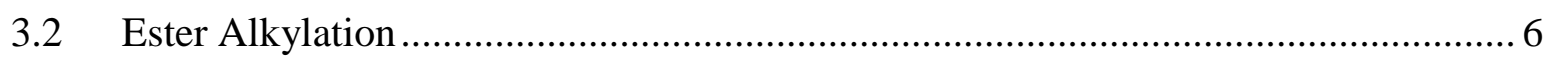

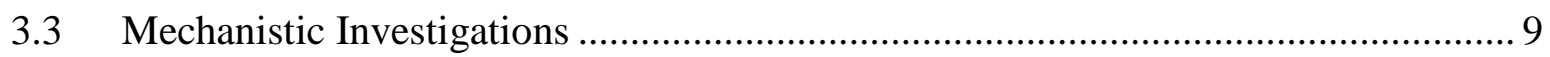

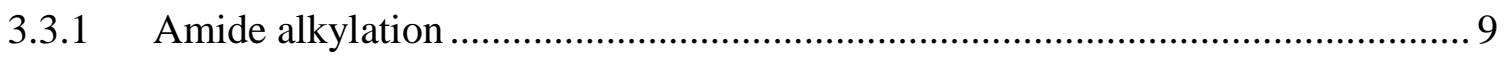

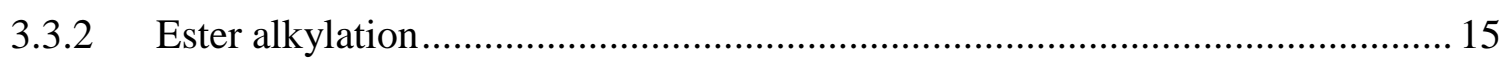

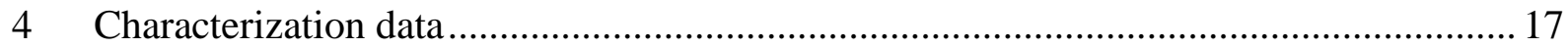

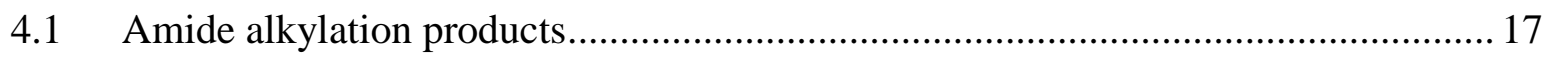

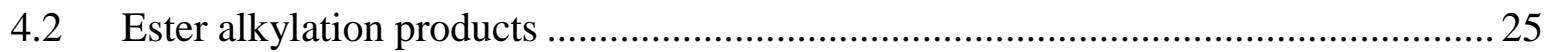

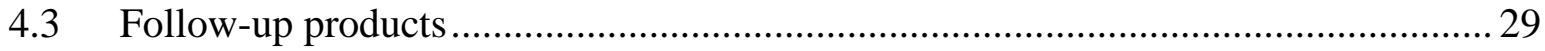

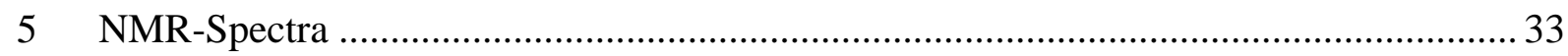

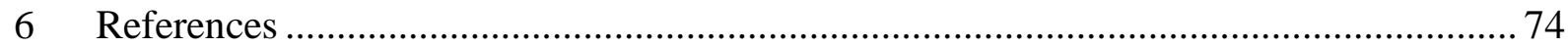




\section{General Methods}

Air- and moisture sensitive reactions were carried out under nitrogen or argon atmosphere using standard Schlenk techniques or a glove box. Dry solvents were obtained from a solvent purification system (activated alumina cartridges) or purchased from Acros. Chemicals were purchased from commercial vendors and used without purification if not noted otherwise. NMR-Spectra were collected at ambient temperature $\left(23^{\circ} \mathrm{C}\right)$ on Varian INOVA 300 (300 $\mathrm{MHz}$ for ${ }^{1} \mathrm{H}, 75 \mathrm{MHz}$ for ${ }^{13} \mathrm{C}$ ) or Bruker Avance III HD $500\left(500 \mathrm{MHz}\right.$ for ${ }^{1} \mathrm{H}, 126$ (125.76) $\mathrm{MHz}$ for ${ }^{13} \mathrm{C}$ ) instruments. Chemical shifts are reported in ppm relative to the residual solvent signal $\left(\mathrm{CDCl}_{3}\right.$ : $\left.7.26 \mathrm{ppm}\left({ }^{1} \mathrm{H}\right), 77.16 \mathrm{ppm}\left({ }^{13} \mathrm{C}\right)\right)$. Coupling constants $(J)$ are reported in $\mathrm{Hz}$ (coupling patterns: $\mathrm{d}=$ doublet, $\mathrm{t}=$ triplet, $\mathrm{q}=$ quartet, $\mathrm{sxt}=$ sextet, $\mathrm{spt}=$ septet, $\mathrm{m}=$ multiplet). GC analyses were carried out on an Agilent 6890N Network GC system equipped with a HP-5 column ( $30 \mathrm{~m} \times 0.32 \mathrm{~mm} \times 0.25 \mu \mathrm{m})$ or on an Agilent $6850 \mathrm{GC}$ system equipped with a Optima17 column ( $30 \mathrm{~m} \times 0.32 \mathrm{~mm} \times 25 \mu \mathrm{m})$. GC-MS analyses were carried out on an Agilent 7890A GC system equipped with a HP-5MS column (30 m x $0.32 \mathrm{~mm}$ x $0.25 \mu \mathrm{m}$ ) and a 5975C inert MSD detector. Flash column chromatography was conducted on MachereyNagel silica gel $60(40-63 \mu \mathrm{m}$ particle size). Elemental analysis was performed on an Elementar Vario El III Instrument. High resolution mass spectra (HRMS) were obtained from a Thermo Fisher Scientific Q-Exactive (Orbitrap) instrument in ESI+ mode.

MTBE $=$ Methyl tert -butyl ether.

DIBAL-H = Diisopropylaluminum hydride.

$t$-BuOK was dried under high vacuum at $70{ }^{\circ} \mathrm{C}$ and stored in a glove box.

The identity and purity of all compounds was characterized by ${ }^{1} \mathrm{H}$ and ${ }^{13} \mathrm{C}$ NMR spectroscopy and GC-MS. Unknown compounds were further characterized by HRMS or elemental analysis. Ambiguous NMR spectra were confirmed by two-dimensional methods.

Compounds 5i, 5m, 5v, 6e, 6g, 7a, 7c, 7d were previously not described in the literature.

Ligands $^{1}$ and Co-complexes ${ }^{2}$ were prepared according to methods previously reported by our group. Representative procedures are also given below.

\section{General procedures}

Typical procedure for ligand preparation: Under inert gas atmosphere a round-bottom flask with gas inlet was charged with a magnetic stirring bar and a solution of the 
corresponding triazine or pyridine diamine $(1 \mathrm{eq})$ in abs. THF $(\sim 0.1 \mathrm{M})$. The solution was cooled to $0{ }^{\circ} \mathrm{C}$ and the corresponding chlorophosphine (2.1 eq) was added dropwise by syringe through a septum maintaining a positive inert gas pressure. Afterwards triethylamine (4 eq) was added dropwise by syringe. The flask was sealed and the reaction was warmed to room temperature and then heated to $60{ }^{\circ} \mathrm{C}$ overnight. After cooling, triethyl ammoniumchloride was allowed to settle and the organic phase was isolated by filtration. The salt cake was washed with THF once and the combined organic phases were concentrated and dried under high vacuum giving the PNP ligand. In most cases no further purification was required. Otherwise, the ligands can be recrystallized in a small amount of hot toluene.

Co-complex preparation. Under inert gas atmosphere (glove box) a Schlenk tube was charged with a magnetic stirring bar and a suspension of $\mathrm{CoCl}_{2}(1 \mathrm{eq})$ in THF $(\sim 0.12 \mathrm{M})$. The PNP-ligand (1 eq) was dissolved in THF $(\sim 0.12 \mathrm{M})$ and added to the stirred suspension of $\mathrm{CoCl}_{2}$ in one portion leading to an instant color change. The Schlenk tube was sealed, removed from the glove box and heated to $60{ }^{\circ} \mathrm{C}$ overnight.

Complexes 1e, 1f were isolated upon concentration in vacuo. Complex 1d precipitated from the reaction mixture and the solvent was concentrated to half volume, the organic phase was removed and the remainder was dried under high vacuum.

Amide alkylation (1 mmol scale). Using a glove box (dry $\mathrm{N}_{2}$ atmosphere), an oven dried 10 $\mathrm{mL}$ top screw vial was charged with a magnetic stirring bar, $t$-BuOK $(1.2 \mathrm{mmol}, 134.4 \mathrm{mg}$, $1.2 \mathrm{eq})$, alcohol (1 mmol, $1.0 \mathrm{eq})$, catalyst $1 \mathrm{e}(1 \mathrm{~mL}$ from a $0.025 \mathrm{M}$ stock solution in THF, $0.025 \mathrm{mmol}, 2.5 \mathrm{~mol} \%)$, amide ( $2 \mathrm{mmol}, 2.0 \mathrm{eq})$ and THF (3.0 mL). The vial was sealed, removed from the glove box and the reaction mixture was heated to $100{ }^{\circ} \mathrm{C}$ (oil bath temperature) for $24 \mathrm{~h}$. The reaction mixture was quenched with water $(1 \mathrm{~mL})$ and extracted with diethyl ether ( $3 \times 10 \mathrm{~mL}$ ). The organic phase was analyzed with GC and GC-MS to monitor consumption of alcohol and product formation. The combined organic phases were dried over $\mathrm{Na}_{2} \mathrm{SO}_{4}$, evaporated and the amide product was purified using a small column of silica gel and pentane/ethyl acetate as the eluent.

As shown for product $\mathbf{5 t}$, the reaction gives the same yields when run on higher scale (10 mmol).

Version without pre-synthesized complex (1 mmol scale reaction): In an analogous manner as described above, a $10 \mathrm{~mL}$ top screw vial was charged with a magnetic stirring bar and $\mathrm{CoCl}_{2}$ (0.025 mmol, $3.25 \mathrm{mg}, 2.5 \mathrm{~mol} \%)$. A solution of the corresponding ligand (0.025 mmol, 9.96 $\mathrm{mg})$ in THF ( $1 \mathrm{~mL})$ was added. After stirring for $2 \mathrm{~min}$, a deep purple solution was obtained. Afterwards, the remaining components were added and the reaction was conducted and 
subjected to work up as described above. Amide 5a was isolated in $81 \%$ yield (143 mg, 0.808 mmol).

Ester alkylation. Using a glove box (dry $\mathrm{N}_{2}$ atmosphere), an oven dried glass tube (38 $\mathrm{mL}$ volume) was charged with a magnetic stirring bar, $t$-BuOK (1.5 mmol, $168 \mathrm{mg}, 1.5 \mathrm{eq})$, alcohol (1 mmol, $1.0 \mathrm{eq})$, catalyst $1 \mathrm{~d}$ ( $31 \mathrm{mg}, 0.05 \mathrm{mmol}, 5 \mathrm{~mol} \%)$, tert-butyl acetate (4 mmol, $4.0 \mathrm{eq})$ and toluene $(1 \mathrm{~mL})$. The tube was sealed with a teflon cap, removed from the glove box and the reaction mixture was heated to $80{ }^{\circ} \mathrm{C}$ (oil bath temperature) for $4 \mathrm{~h}$. The reaction mixture was quenched with water $(1 \mathrm{~mL})$ and extracted with diethyl ether $(3 \mathrm{x} 10$ $\mathrm{mL}$ ). The organic phase was analyzed with GC and GC-MS to monitor consumption of alcohol and product formation. The combined organic phases were dried over $\mathrm{Na}_{2} \mathrm{SO}_{4}$, evaporated and the ester product was purified using flash column chromatography.

Note: Since the reaction mixture gets viscous during the reaction, efficient stirring is necessary. Therefore, a reaction tube with higher diameter is beneficial.

\section{Screening reactions}

In order to find the optimal reaction conditions for ester and amide alkylation, the following reactions were investigated.

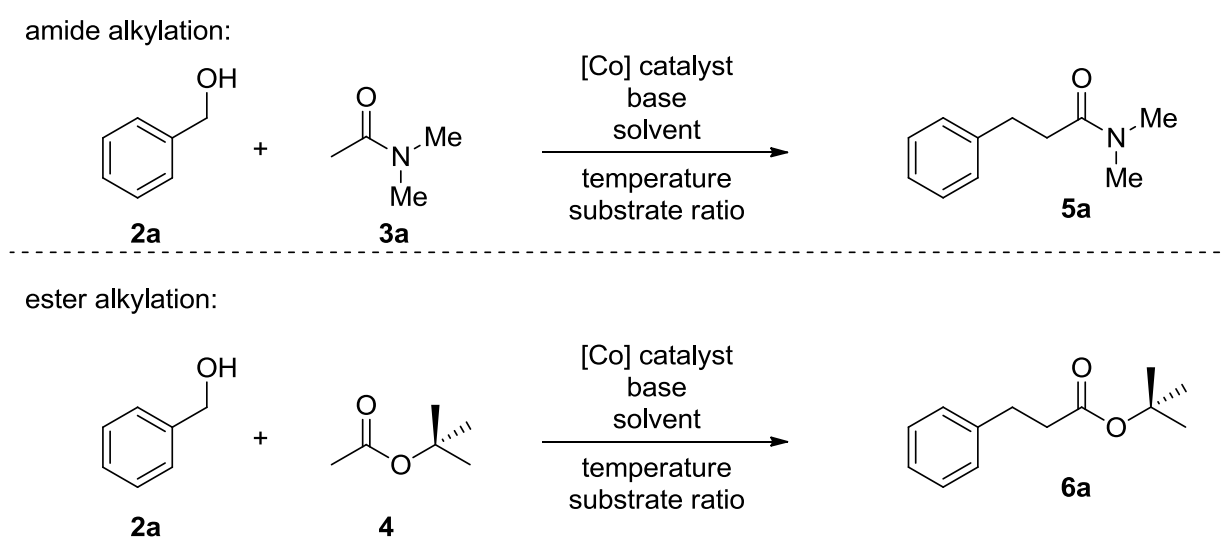

Typical screening procedure: Using a nitrogen-filled glove box, a $10 \mathrm{~mL}$ top-screw vial was charged with a magnetic stirring bar, the corresponding starting materials, base, catalyst and solvent. The vial was sealed, removed from the glove box and the reaction mixture was immersed into a pre-heated oil bath. Afterwards, the reaction was cooled, water $(1 \mathrm{~mL})$, diethyl ether $(5 \mathrm{~mL})$ and dodecane (GC-standard) were added. After shaking, an aliquot of the organic phase was analyzed by GC-FID to determine the yield based on the internal standard method. For further details, see the table footnotes.

For catalyst screening, see article. 


\subsection{Amide alkylation}

Table S 1. Solvent Screening

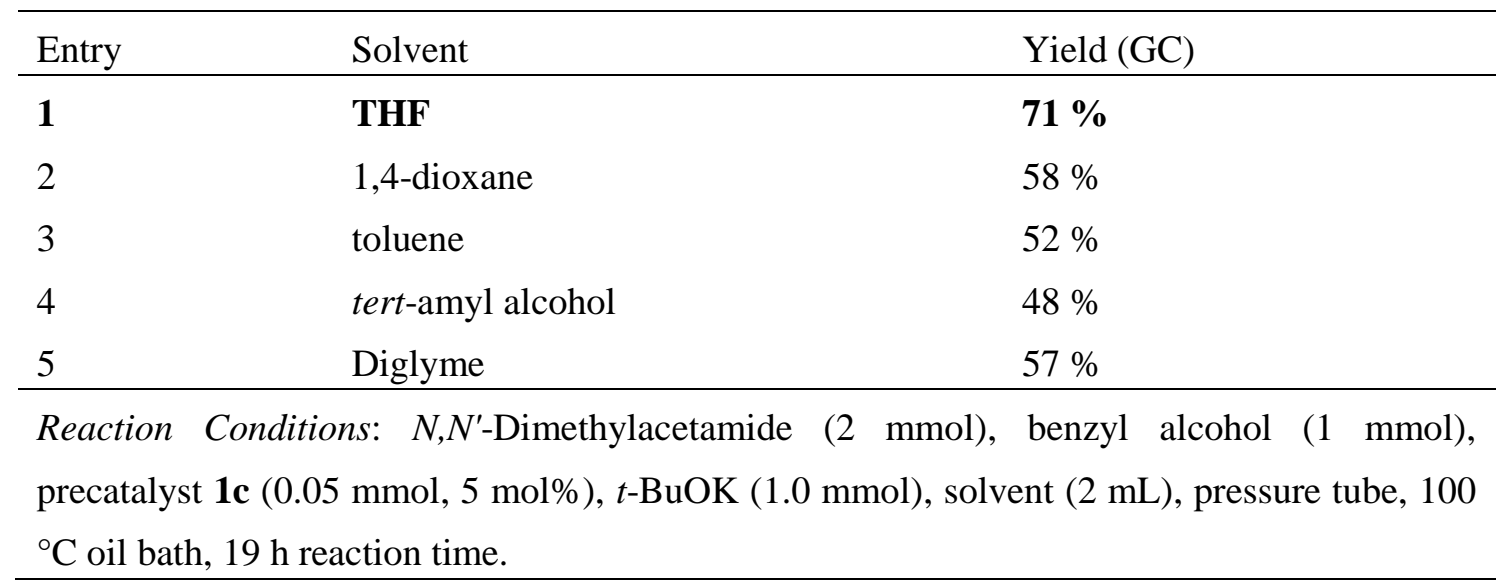

Table S 2. Base Screening

\begin{tabular}{lll}
\hline Entry & Base & Yield (GC) \\
\hline 1 & $t$-BuOLi & $28 \%$ \\
2 & $t$-BuONa & $63 \%$ \\
$\mathbf{3}$ & $\boldsymbol{t}$-BuOK & $\mathbf{6 5} \%$ \\
4 & $\mathrm{KOH}$ & $0 \%$ \\
5 & $\mathrm{KH}$ & $45 \%$ \\
6 & $\mathrm{Cs}_{2} \mathrm{CO}_{3}$ & $0 \%$ \\
7 & $\mathrm{KHMDS}^{2} \quad \mathrm{~N}, N^{\prime}$-Dimethylacetamide & $(2 \mathrm{mmol})$, benzyl alcohol $(1 \mathrm{mmol})$, \\
Reaction & Conditions & $23 \%$ \\
precatalyst 1c $(0.05 \mathrm{mmol}, 5 \mathrm{~mol} \%)$, base $(1.0 \mathrm{mmol}), \mathrm{THF}(2 \mathrm{~mL})$, pressure tube, $100{ }^{\circ} \mathrm{C}$ oil \\
bath, 19 h reaction time.
\end{tabular}


Table S 3. Base Amount Screening

\begin{tabular}{lll}
\hline Entry & Base amount [eq.] & Yield (GC) \\
\hline 1 & 0.1 & $0 \%$ \\
2 & 0.2 & $0 \%$ \\
3 & 0.5 & $25 \%$ \\
4 & 1.0 & $55 \%$ \\
$\mathbf{5}$ & $\mathbf{1 . 2}$ & $\mathbf{6 6} \%$ \\
6 & 1.5 & $65 \%$ \\
7 & 1.7 & $69 \%$ \\
8 & 2.0 & $69 \%$ \\
\hline Reaction & Conditions: $N, N^{\prime}$-Dimethylacetamide $(2 \mathrm{mmol})$, benzyl alcohol $(1$ mmol $)$, \\
precatalyst 1c $(0.05$ mmol, 5 mol\%), $t$-BuOK $(0.1-2.0 \mathrm{mmol})$, THF $(3 \mathrm{~mL})$, pressure tube, \\
$10{ }^{\circ}$ C oil bath, $19 \mathrm{~h}$ reaction time.
\end{tabular}

Table S 4. Screening of substrate ratio

\begin{tabular}{lll}
\hline Entry & $\mathrm{Me}_{2} \mathrm{NAc}: \mathrm{BnOH}$ ratio & Yield $(\mathrm{GC})$ \\
\hline 1 & $1.0: 1.0$ & $58 \%$ \\
2 & $1.5: 1.0$ & $64 \%$ \\
$\mathbf{3}$ & $\mathbf{2 . 0}: \mathbf{1 . 0}$ & $\mathbf{6 8 \%}$ \\
4 & $1.0: 1.5$ & $39 \%$ \\
5 & $1.0: 2.0$ & $35 \%$ \\
\hline
\end{tabular}

Reaction Conditions: $N, N^{\prime}$-Dimethylacetamide (1-2 mmol), benzyl alcohol (1-2 mmol), precatalyst 1c $(0.05 \mathrm{mmol}, 5 \mathrm{~mol} \%), t$-BuOK $(1.2 \mathrm{mmol})$, THF $(2 \mathrm{~mL})$, pressure tube, $100{ }^{\circ} \mathrm{C}$ oil bath, $19 \mathrm{~h}$ reaction time.

Table S 5. Temperature Screening

\begin{tabular}{|c|c|c|}
\hline Entry & Temperature & Yield (GC) \\
\hline 1 & $60^{\circ} \mathrm{C}$ & $40 \%$ \\
\hline 2 & $80{ }^{\circ} \mathrm{C}$ & $65 \%$ \\
\hline 3 & $100{ }^{\circ} \mathrm{C}$ & $67 \%$ \\
\hline \multicolumn{3}{|c|}{$\begin{array}{l}\text { Reaction Conditions: N,N'-Dimethylacetamide }(2 \mathrm{mmol}) \text {, benzyl alcohol }(1 \mathrm{mmol}) \text {, } \\
\text { precatalyst } 1 \mathrm{c}(0.05 \mathrm{mmol}, 5 \mathrm{~mol} \%), t \text {-BuOK }(1.2 \mathrm{mmol}) \text {, THF }(2 \mathrm{~mL}) \text {, pressure tube, } 100{ }^{\circ} \mathrm{C} \\
\text { oil bath, } 19 \mathrm{~h} \text { reaction time. }\end{array}$} \\
\hline
\end{tabular}




\subsection{Ester Alkylation}

Table S 6. Solvent Screening

\begin{tabular}{lll}
\hline Entry & Solvent & Yield (GC) \\
\hline 1 & THF & $24 \%$ \\
$\mathbf{2}$ & Toluene & $\mathbf{4 0} \%$ \\
3 & tert-amyl alcohol & $8 \%$ \\
4 & 1,4 -dioxane & $27 \%$ \\
5 & Diglyme & $13 \%$ \\
\hline
\end{tabular}

Reaction Conditions: tert-butyl acetate $(2.0 \mathrm{mmol})$, benzyl alcohol $(1.0 \mathrm{mmol})$, solvent $(2$ $\mathrm{mL}), t$-BuOK $(1 \mathrm{mmol})$, precatalyst $1 \mathrm{c}(0.05 \mathrm{mmol}, 5 \mathrm{~mol} \%), 80{ }^{\circ} \mathrm{C}$ oil bath, sealed tube, $20 \mathrm{~h}$

Table S 7. Base Screening

\begin{tabular}{lll}
\hline Entry & Base (1.0 eq.) & Yield (GC) \\
\hline 1 & $t$-BuOLi & $n / d$ \\
2 & $t$-BuONa & $37 \%$ \\
$\mathbf{3}$ & $t$-BuOK & $\mathbf{3 8} \%$ \\
4 & $\mathrm{KOH}$ & $n / d$ \\
5 & $\mathrm{KH}$ & $18 \%$ \\
6 & $\mathrm{Cs}_{2} \mathrm{CO}_{3}$ & $n / d$ \\
7 & $\mathrm{KHMDS}$ & $15 \%$ \\
\hline
\end{tabular}

Reaction Conditions: tert-butyl acetate $(2.0 \mathrm{mmol})$, benzyl alcohol $(1.0 \mathrm{mmol})$, toluene (2 $\mathrm{mL})$, base $(1 \mathrm{mmol})$, precatalyst $1 \mathrm{c}(0.05 \mathrm{mmol}, 5 \mathrm{~mol} \%), 70{ }^{\circ} \mathrm{C}$, sealed tube, $20 \mathrm{~h}$ $\mathrm{n} / \mathrm{d}=$ not detected 
Table S 8. Base Amount Screening

\begin{tabular}{lll}
\hline Entry & Base amount (eq) & Yield (GC) \\
\hline 1 & 0.10 & $n / d$ \\
2 & 0.20 & $n / d$ \\
3 & 0.50 & $15 \%$ \\
4 & 1.00 & $43 \%$ \\
5 & 1.20 & $53 \%$ \\
$\mathbf{6}$ & $\mathbf{1 . 5 0}$ & $\mathbf{6 0} \%$ \\
7 & 1.70 & $57 \%$ \\
8 & 2.00 & $53 \%$ \\
\hline
\end{tabular}

Reaction Conditions: tert-butyl acetate $(2.0 \mathrm{mmol})$, benzyl alcohol $(1.0 \mathrm{mmol})$, toluene $(2$ $\mathrm{mL})$, t-BuOK (0.1-2.0 mmol), precatalyst $1 \mathrm{c}(0.05 \mathrm{mmol}, 5 \mathrm{~mol} \%), 70{ }^{\circ} \mathrm{C}$, sealed tube, $20 \mathrm{~h}$ $n / d=$ not detected

Table S 9. Screening of substrate ratio

\begin{tabular}{lll}
\hline Entry & $t$-BuOAc/BnOH ratio & Yield $(\mathrm{GC})$ \\
\hline 1 & $2.0: 1.0$ & $55 \%$ \\
$\mathbf{2}$ & $\mathbf{4 . 0}: \mathbf{1 . 0}$ & $\mathbf{9 0} \%$ \\
3 & $6.0: 1.0$ & $90 \%$ \\
4 & Neat ester $(1.5 \mathrm{~mL})$ & $52 \%$ \\
\hline
\end{tabular}

Reaction Conditions: tert-butyl acetate (2.0-1.0 mmol), benzyl alcohol (1.5-1.0 mmol), toluene $(2 \mathrm{~mL})$, t-BuOK $(1.5 \mathrm{mmol})$, precatalyst $1 \mathbf{d}(0.05 \mathrm{mmol}, 5 \mathrm{~mol} \%), 70{ }^{\circ} \mathrm{C}$, sealed tube, $20 \mathrm{~h}$.

(Screening with optimized catalyst 1d)

The reaction can be run within a temperature range of $70-100{ }^{\circ} \mathrm{C}$ without high variations in yield (Table S 10). $80^{\circ} \mathrm{C}$ was chosen eventually to account for a broad scope.

Table S 10. Screening of temperature

\begin{tabular}{lll}
\hline Entry & Temperature & Isolated yield of $6 \mathbf{a}$ \\
\hline 1 & $70{ }^{\circ} \mathrm{C}$ & $70 \%$ \\
2 & $100{ }^{\circ} \mathrm{C}$ & $71 \%$ \\
\hline Reaction Conditions: tert-butyl acetate $(4 \mathrm{mmol})$, benzyl alcohol $(1 \mathrm{mmol})$, toluene $(1 \mathrm{~mL}), t-$ \\
BuOK $(1.5 \mathrm{mmol})$, precatalyst $\mathbf{1 d}(0.05 \mathrm{mmol}, 5 \mathrm{~mol} \%), 70{ }^{\circ} \mathrm{C}$, sealed tube, $4 \mathrm{~h}$. \\
\hline
\end{tabular}

The reaction was found to be complete in 4 hours. For the following products, the isolated yields were compared for different reaction times ( $4 \mathrm{~h}$ vs. $12 \mathrm{~h}$ ) and catalyst loadings ( 3 mol\% 
vs. $5 \mathrm{~mol} \%$,). No significant variations were detected and $4 \mathrm{~h}$ reaction time was chosen eventually (Table S 11).

Table S 11. Comparison of reaction conditions.

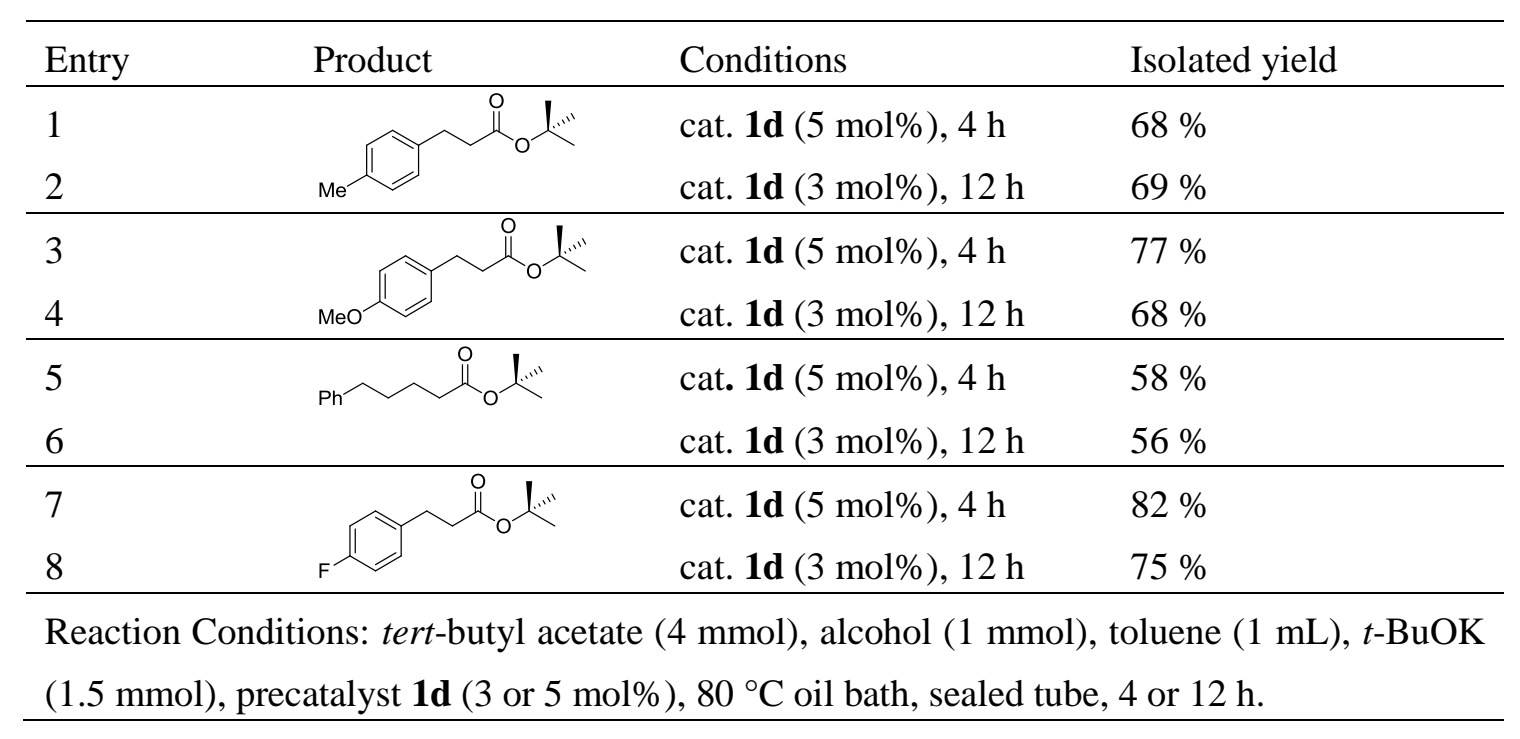




\subsection{Mechanistic Investigations}

\subsubsection{Amide alkylation}

\subsubsection{Synthesis of $N, N$-dimethylcinnamamide}

In order to obtain deeper mechanistic insight into the alkylation reaction, we first prepared $N, N$-dimethylcinnamamide (S-1, Scheme $\mathrm{S} 1$, see also synthesis section) which is believed to be a central intermediate in the catalytic cycle.

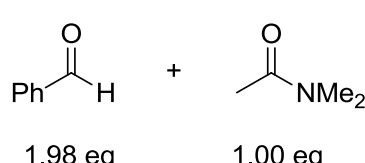

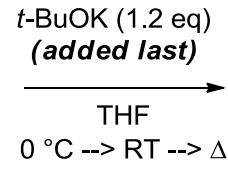

Scheme S 1. Preparation of N,N-dimethylcinnamamide.

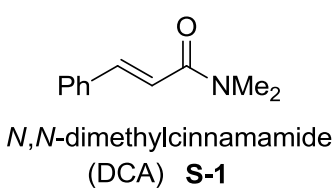

(DCA) S-1

During preparation, we noticed formation of the di-amide S-2 (Scheme S 2), which is derived from 1,4-addition of $N, N$-dimethylacetamide to DCA. S-2 was obtained exclusively when benzaldehyde was added dropwise to the anion of $N, N$-dimethylacetamide (prepared from $N, N$-dimethylacetamide and $t$-BuOK in THF, Scheme S 2).

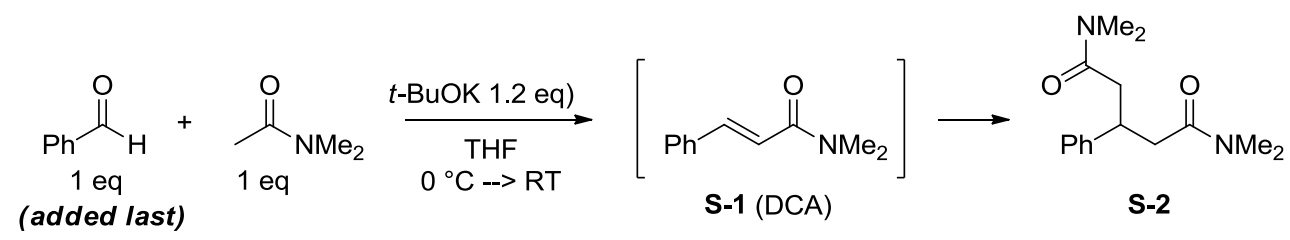

Scheme S 2. Attempted synthesis of N,N-dimethylcinnamamide.

The crude reaction mixture of all prepared amide substrates was analyzed by GC and in general only low amounts of side-products occurred. This result suggested that the concentration of unsaturated intermediate [DCA (S-1) for the model reaction] in the reaction mixture must be low (see also Figure $\mathrm{S} 2$ for a typical gas-chromatogram after reaction end).

3.3.1.2 Time-conversion-plot for the model reaction (amide alkylation)

The time-conversion-plot of the model-reaction of the amide alkylation reaction is depicted in Figure $\mathrm{S}$ 1. Yields and conversions were obtained by reaction monitoring by GC with dodecane as internal standard. 


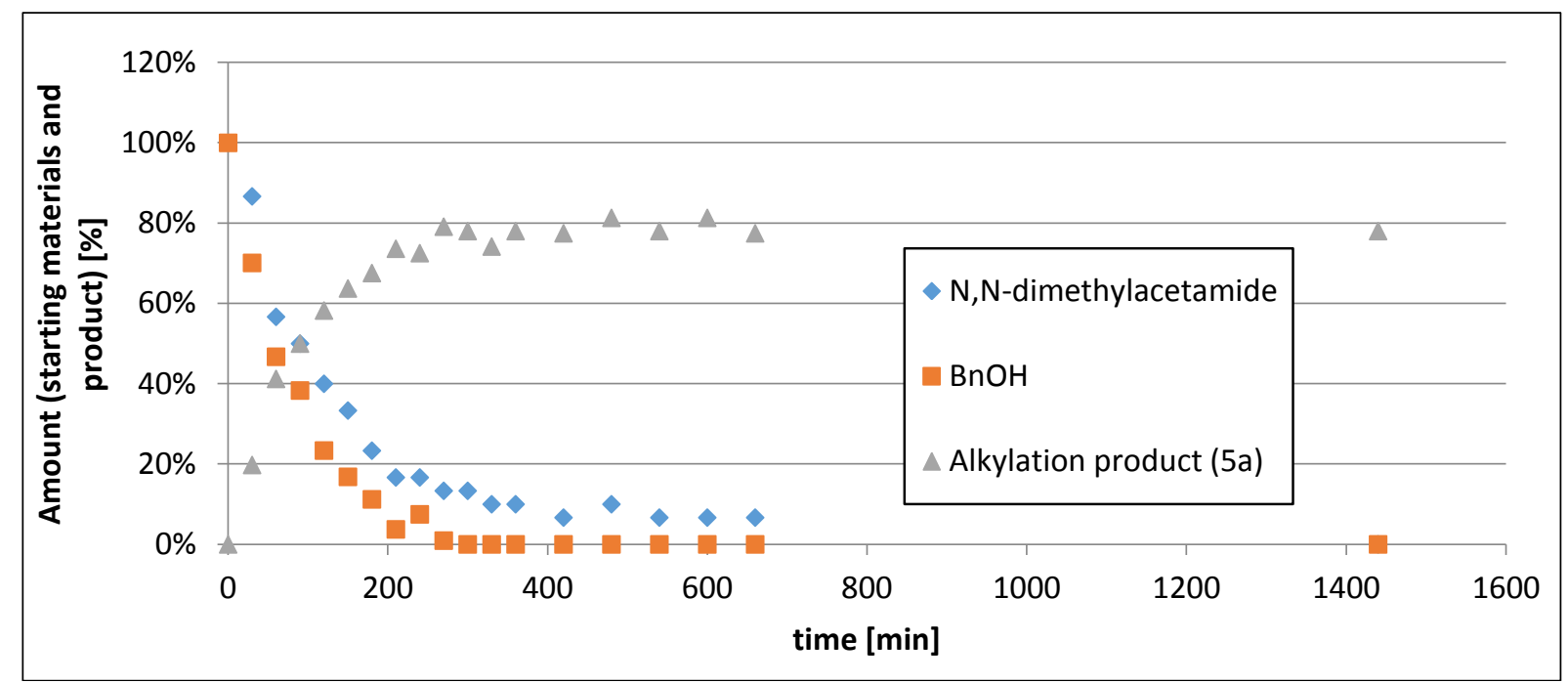

Figure S 1. Time-conversion plot of the amide alkylation reaction.

\section{Reaction start}

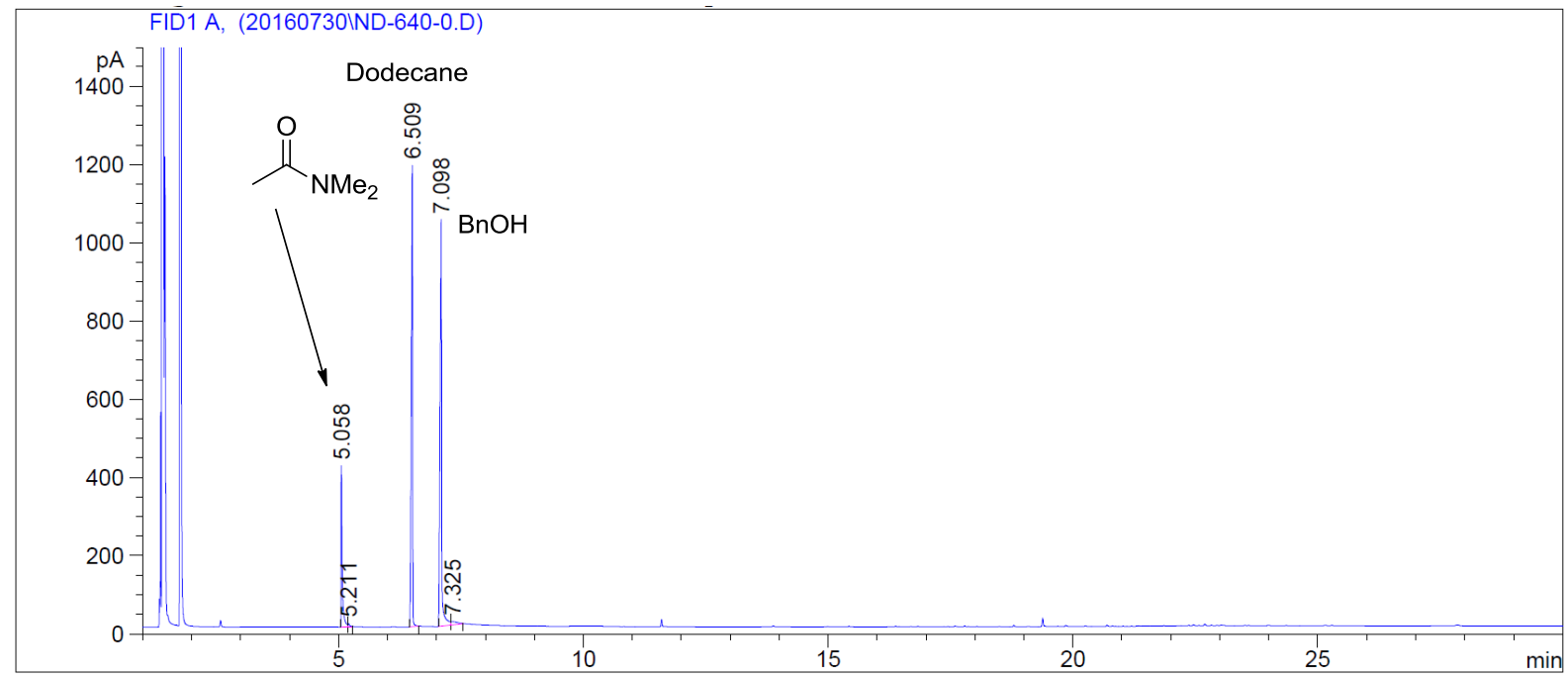

Reaction end (24 h)

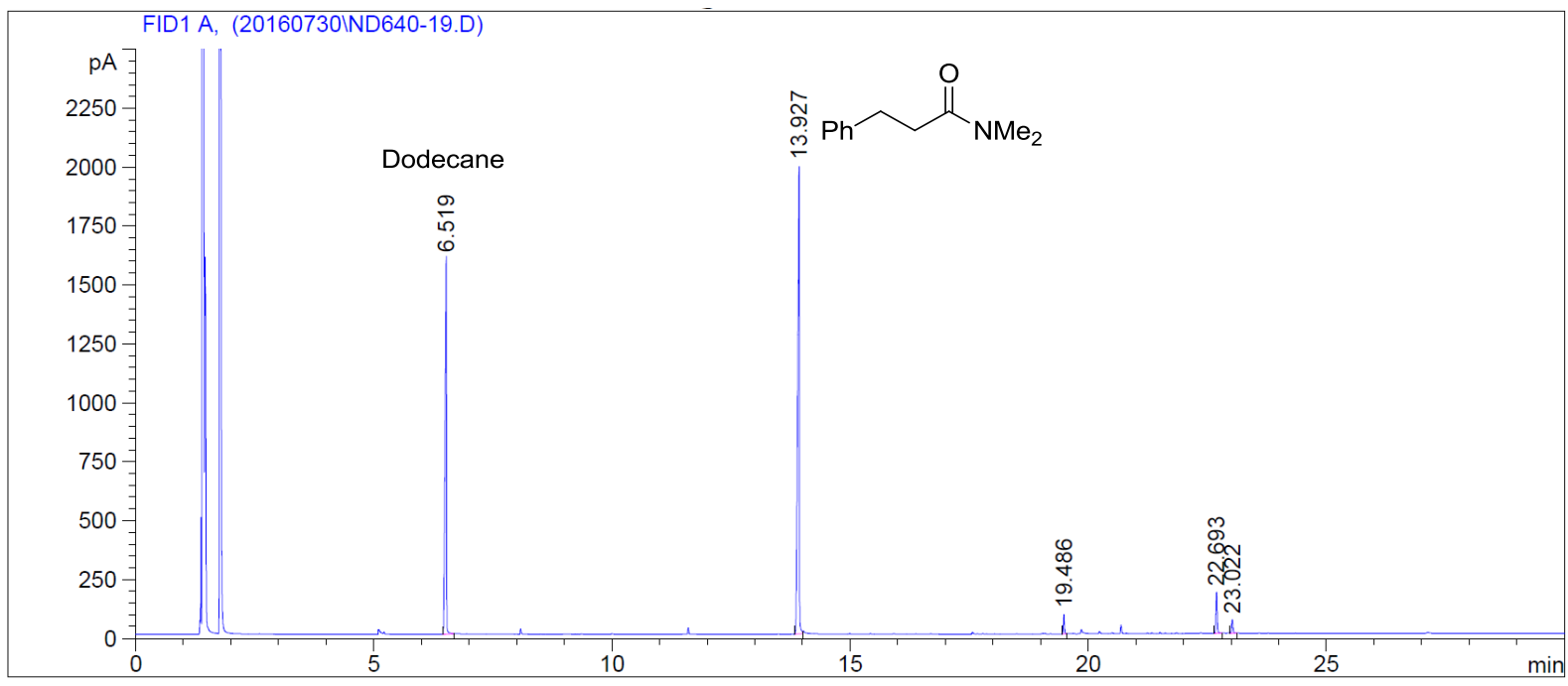

Figure S 2. Gas-chromatograms of the model reaction at reaction start and reaction end. 
The following conclusions were drawn:

- No induction period was observed

- No $N, N$-dimethylcinnamamide intermediate was detected during the reaction which means the oxidation of alcohol is rate-determining and reduction proceeds relatively fast.

- No significant amount of side-products was observed.

- Alcohol conversion and product formation correlate.

\subsubsection{Investigation of the oxidation step}

For an Ir-catalyst, the transfer hydrogenation between benzyl alcohol and 3,3-dimethylbut-1ene is described in the literature as the only mechanistic work. When this reaction was conducted with the cobalt catalyst, no significant conversion of benzyl alcohol was observed with or without base (Scheme S 3).

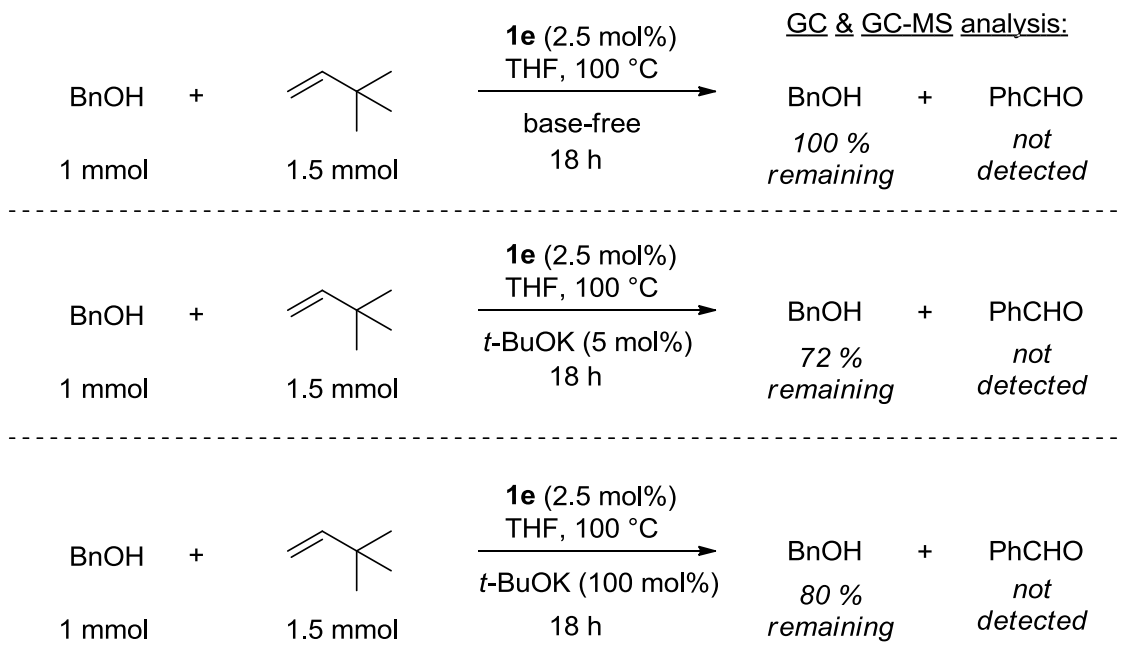

Scheme S 3. Investigation of transfer hydrogenation.

\subsubsection{Concerted oxidation/reduction}

When the reaction between the proposed intermediate $N, N$-dimethylcinnamamide and benzyl alcohol under catalytic conditions was monitored, another side-product accumulated in significant amount. The product was identified by GC-MS as the 1,4-addition product between the intermediate $N, N$-dimethylcinnamamide and the hydrogenation product. This observation further supports the assumption that the reduction of the $\alpha, \beta$-unsaturated intermediate proceeds before competition reactions take place since only very low amounts of this side-product are seen in the catalytic reaction (cf. the gas-chromatogram in Figure S 3 with Figure S 4). 


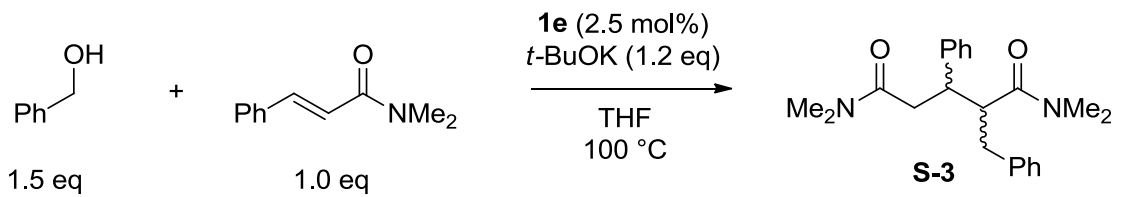

Scheme S 4. Attempted transfer hydrogenation between benzyl alcohol and intermediate.

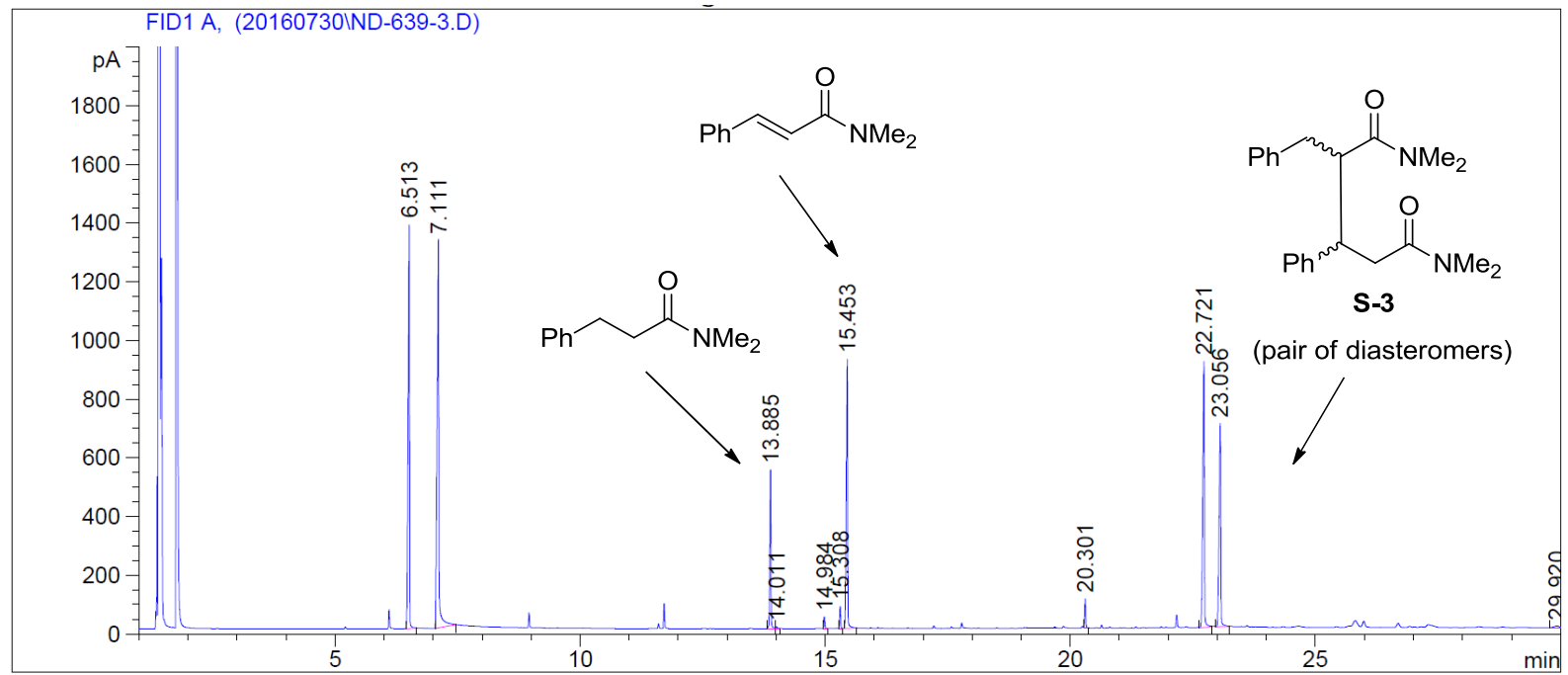

Figure S 3. Gas-chromatogram of the reaction depicted in Scheme S 4 after $1.5 \mathrm{~h}$.

Comparison with catalytic reaction:

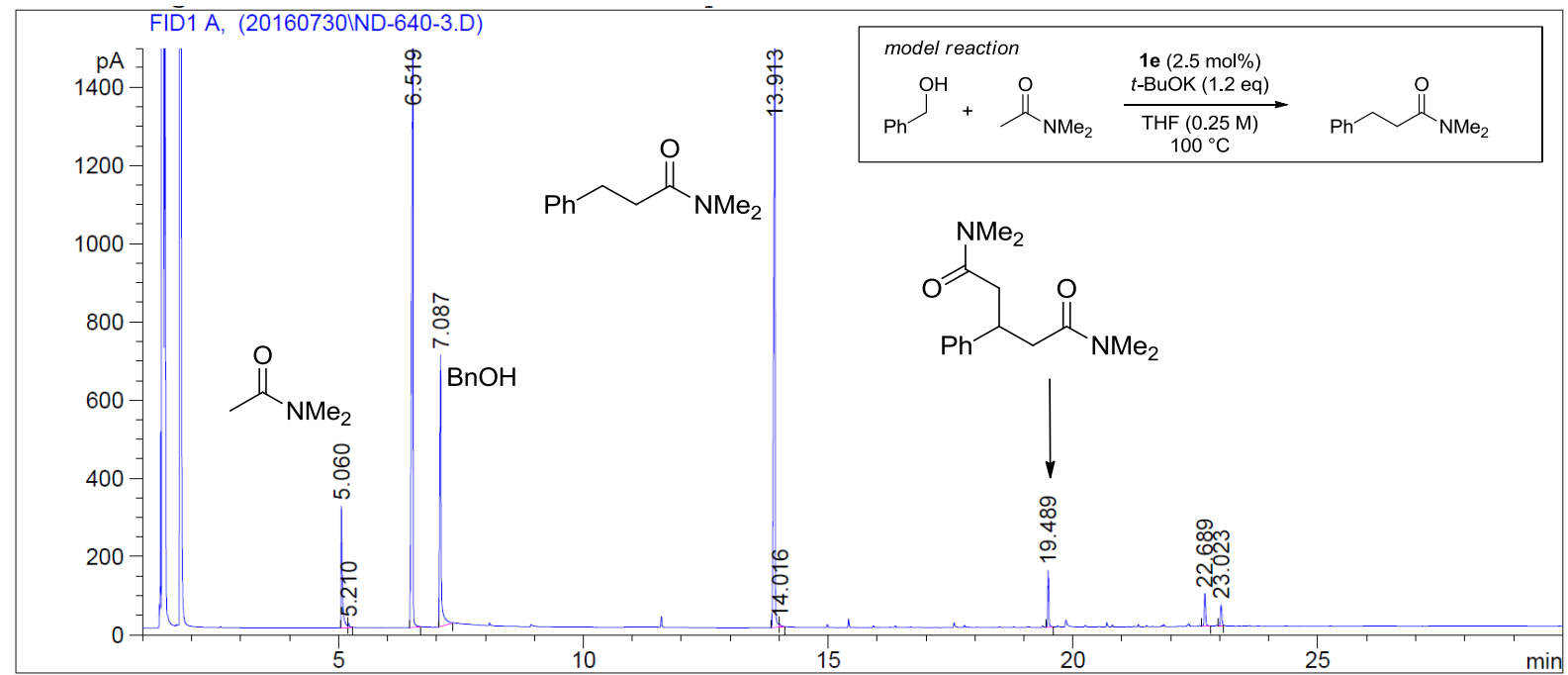

Figure S 4. Gas-chromatogram of the model reaction after $1.5 \mathrm{~h}$.

Possible 1,4-addition products were prepared independently to verify GC-measurements (Scheme S 5). 


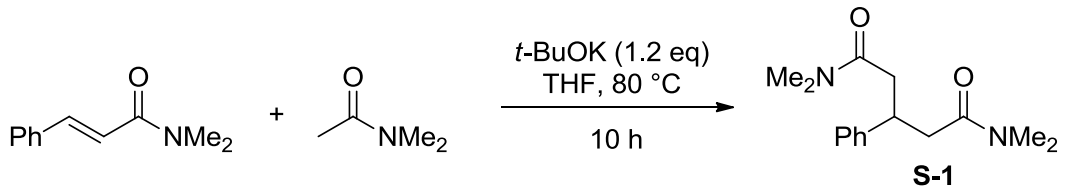

GC-MS: Same retention time and mass-spectrum as in the catalytic experiment. Verified by HRMS

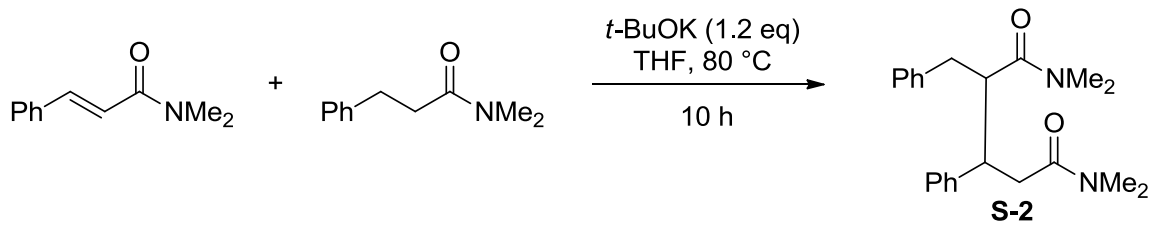

GC-MS: Same retention time and mass-spectrum as in the catalytic experiment.

Verified by HRMS

Scheme S 5. Control experiments to verify possible 1,4-addition side-products.

\subsubsection{Hydrogenation experiments}

DCA (S-1) was subjected to hydrogenation under catalytic conditions. If hydrogen equivalents are not obtained from the rate-limiting alcohol oxidation, but from molecular hydrogen, it was envisioned that fast reduction would limit 1,4-addition side-reactions.
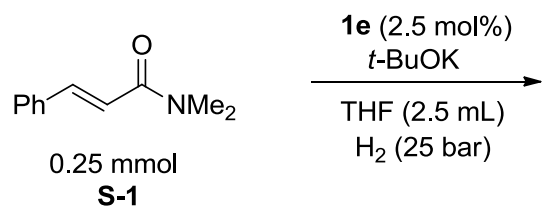<smiles>CNC(=O)CCc1ccccc1</smiles>

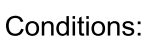

(determined by GC with dodecane as internal standard)

Scheme S 6. Hydrogenation experiments.

Hydrogenation proceeded even within 30 min (entry 6 in Scheme S 6) and the 1,4-addition product was not obtained.

\subsubsection{Syntheses}

\section{$N, N$-dimethylcinnamamide $(\boldsymbol{S}-\mathbf{1})$}<smiles>CN(C)C(=O)/C=C/c1ccccc1</smiles>

To a stirred solution of benzaldehyde $(98.9 \mathrm{mmol}, 10 \mathrm{~mL}, 1.98 \mathrm{eq})$ and $\mathrm{N}, \mathrm{N}$ dimethylacetamide $(50.0 \mathrm{mmol}, 4.65 \mathrm{~mL}, 1.00 \mathrm{eq})$ in THF $(200 \mathrm{~mL})$ was added $t$-BuOK (60 mmol, $6.72 \mathrm{~g}, 1.2 \mathrm{eq})$ in portions at $0{ }^{\circ} \mathrm{C}$. The reaction mixture was warmed to $\mathrm{rt}$ and heated 
to reflux for 1 hour. After cooling, water was added until a clear solution was obtained. The reaction mixture was then concentrated and partitioned between water $(50 \mathrm{~mL})$ and $\mathrm{CH}_{2} \mathrm{Cl}_{2}$ (100 mL). After separation of the phases, the aqueous phase was extracted with $\mathrm{CH}_{2} \mathrm{Cl}_{2}(2 \mathrm{x}$ $100 \mathrm{~mL}$ ). The combined organic phase was dried over $\mathrm{Na}_{2} \mathrm{SO}_{4}$, concentrated and purified by column chromatography (silica gel, pentane/ethyl acetate $1: 1 \rightarrow 1: 2$ ) to give the title compound as a crystalline white solid (2.98 g, $17.0 \mathrm{mmol}, 34 \%)$.

${ }^{1} \mathbf{H}$ NMR $\left(500 \mathrm{MHz}, \mathrm{CDCl}_{3}\right) \delta=7.67(\mathrm{~d}, J=15.26 \mathrm{~Hz}, 1 \mathrm{H}), 7.50-7.55$ (m, $\left.2 \mathrm{H}\right), 7.31-7.40$ (m, $3 \mathrm{H}), 6.89$ (d, J=15.26 Hz, $1 \mathrm{H}$ ), 3.17 (s, $3 \mathrm{H}$ ), 3.07 (s, $3 \mathrm{H}) \mathrm{ppm}$.

${ }^{13}$ C NMR $\left(126 \mathrm{MHz}, \mathrm{CDCl}_{3}\right) \delta=166.8,142.5,135.5,129.7,128.9,127.9,117.5,37.6,36.1$ ppm.

MS (EI, $70 \mathrm{eV}) \mathrm{m} / z: 175.1\left(\mathrm{M}^{+}\right), 131.1,103.1,77.1,51.1$.

The spectroscopic data correspond with those reported in the literature. ${ }^{3}$

Note: When the reactants were added in a different order $(N, N$-dimethylacetamide, $t$-BuOK, then dropwise addition of benzaldehyde (1 eq)), the corresponding 1,4-addition product $\mathbf{S}-2$ was obtained as monitored by GC-MS $(\mathrm{m} / \mathrm{z} 262.1)$.

\section{Confirmation of 1,4-addition products:}

2-benzyl- $N^{1}, N^{1}, N^{5}, N^{5}$-tetramethyl-3-phenylpentanediamide<smiles>CNC(=O)CC(c1ccccc1)C(Cc1ccccc1)C(=O)O</smiles>

Under nitrogen atmosphere (glove box), $N, N$-dimethylcinnamamide $(0.5 \mathrm{mmol}, 87.5 \mathrm{mg}, 1$ eq), $N, N$-dimethyl-3-phenylpropanamide $(0.75 \mathrm{mmol}, 133 \mathrm{mg}, 1.5 \mathrm{eq}), t$-BuOK $(67 \mathrm{mg}, 0.6$ mmol, $1.2 \mathrm{eq})$ and THF ( $3 \mathrm{~mL})$ were mixed in a $10 \mathrm{~mL}$ vial. The vial was sealed and the reaction mixture was heated with a $80{ }^{\circ} \mathrm{C}$ oil bath for $10.5 \mathrm{~h}$. The reaction was quenched with water $(2 \mathrm{~mL})$ and extracted with ethyl acetate $(3 \mathrm{x} 10 \mathrm{~mL})$ and concentrated. The crude product was analyzed by GC-MS and LC-HRMS. The above 1,4-addition product was observed at the same retention time as in the catalytic reaction and the identity was further confirmed by HRMS.

GC-MS (EI, $70 \mathrm{eV}) \mathrm{m} / \mathrm{z}: 352.2\left(\mathrm{M}^{+}\right), 308.2,266.1,216.1,176.1,131.1,115.1,103.1,91.1$, 72.1 .

HRMS (ESI+): $m / z$ calcd. for $\left[\mathrm{C}_{22} \mathrm{H}_{28} \mathrm{~N}_{2} \mathrm{O}_{2}+\mathrm{H}\right]^{+} 353.22235$; found: 353.22134 . 
$N^{1}, N^{1}, N^{5}, N^{5}$-tetramethyl-3-phenylpentanediamide<smiles>CNC(=O)CC(CC(=O)NC)c1ccccc1</smiles>

Under nitrogen atmosphere (glove box), $N, N$-dimethylcinnamamide $(0.5 \mathrm{mmol}, 87.5 \mathrm{mg}, 1$ eq), $N, N$-dimethylacetamide ( $1 \mathrm{mmol}, 93 \mu \mathrm{L}, 2 \mathrm{eq}), t$-BuOK (67 mg, $0.6 \mathrm{mmol}, 1.2 \mathrm{eq}$ ) and THF ( $3 \mathrm{~mL}$ ) were mixed in a $10 \mathrm{~mL}$ vial. The vial was sealed and the reaction mixture was heated with a $80{ }^{\circ} \mathrm{C}$ oil bath for $10.5 \mathrm{~h}$. The reaction was quenched with water $(2 \mathrm{~mL})$ and extracted with ethyl acetate $(3 \mathrm{x} 10 \mathrm{~mL})$ and concentrated. The crude product was analyzed by GC-MS and LC-HRMS. The above 1,4-addition product was observed at the same retention time as in the catalytic reaction and the identity was further confirmed by HRMS.

GC-MS (EI, $70 \mathrm{eV}) \mathrm{m} / z: 262.1\left(\mathrm{M}^{+}\right), 218.1,190.1,176.1,131.0,103.0,87.1,72.0$.

HRMS (ESI+): $m / z$ calcd. for $\left[\mathrm{C}_{15} \mathrm{H}_{22} \mathrm{~N}_{2} \mathrm{O}_{2}+\mathrm{H}\right]^{+} 263.17540$; found: 263.17462 .

\subsubsection{Ester alkylation}

\subsubsection{Reaction monitoring}

The reaction of the ester alkylation (model reaction) was monitored by GC with dodecane as internal standard (Scheme S 7 and Figure S 5).

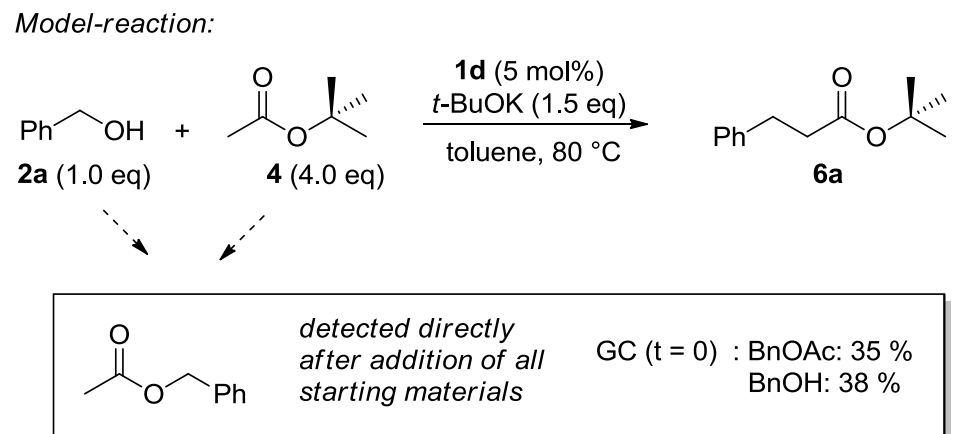

Scheme S 7. Model reaction for ester alkylation and outcome of analysis of the reaction directly after addition of starting materials.

An aliquot (500 $\mu \mathrm{L}$ from a $2 \mathrm{mmol}$ scale reaction) was removed from the reaction mixture immediately after addition of tert-butyl acetate. The aliquot was quenched with water and extracted with diethyl ether.

GC-FID and GC-MS analysis of the $\mathrm{t}=0$ min reaction sample showed a significant amount of benzyl acetate which was confirmed by GC-MS and by comparison of the retention time of an authentic sample.

This result indicates that transesterification products undergo resolution by alcohol exchange with $t$-BuOH followed by consumption of the primary alcohol in the catalytic alkylation reaction. The catalytic reaction gives one single product after 1-4 $\mathrm{h}$. 


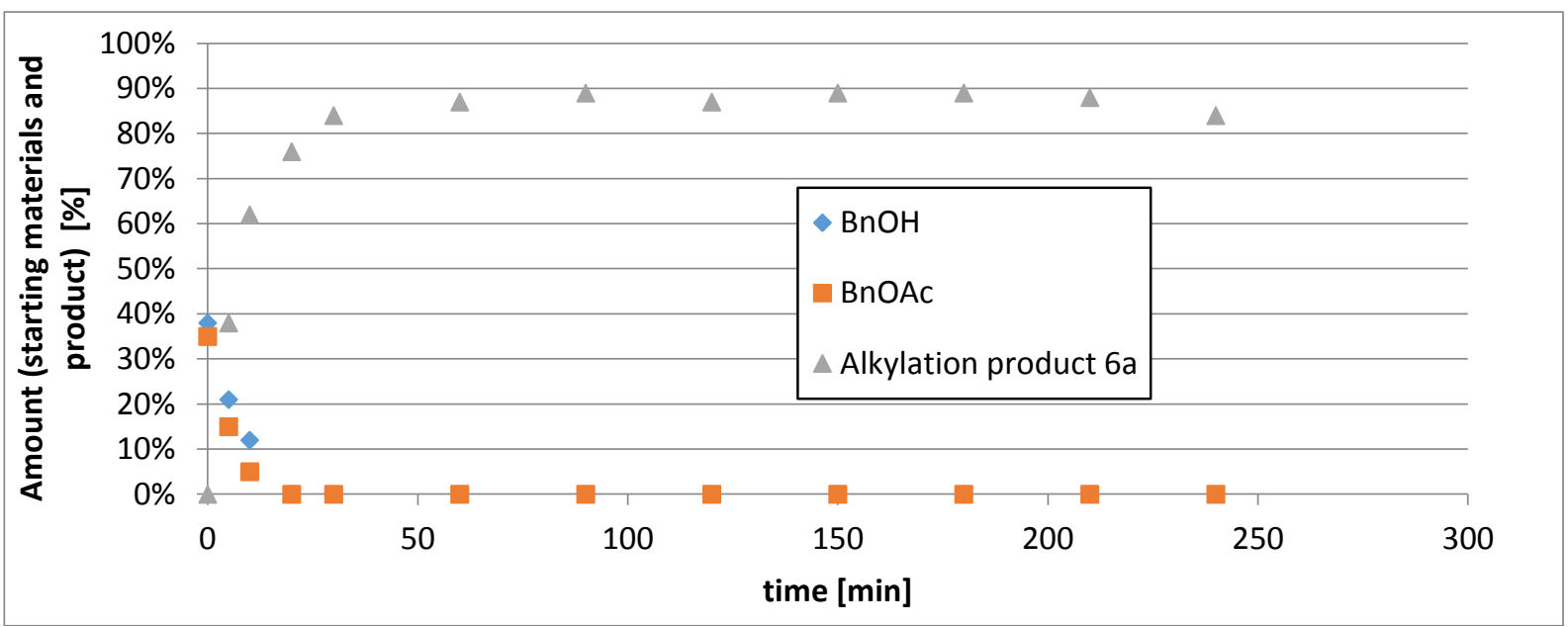

Figure S 5. Time-conversion plot for the catalytic ester alkylation (model reaction).

\subsubsection{Control reactions}

The corresponding transesterification products were subjected to the reaction conditions to prove the shift towards the tert-butyl ester.

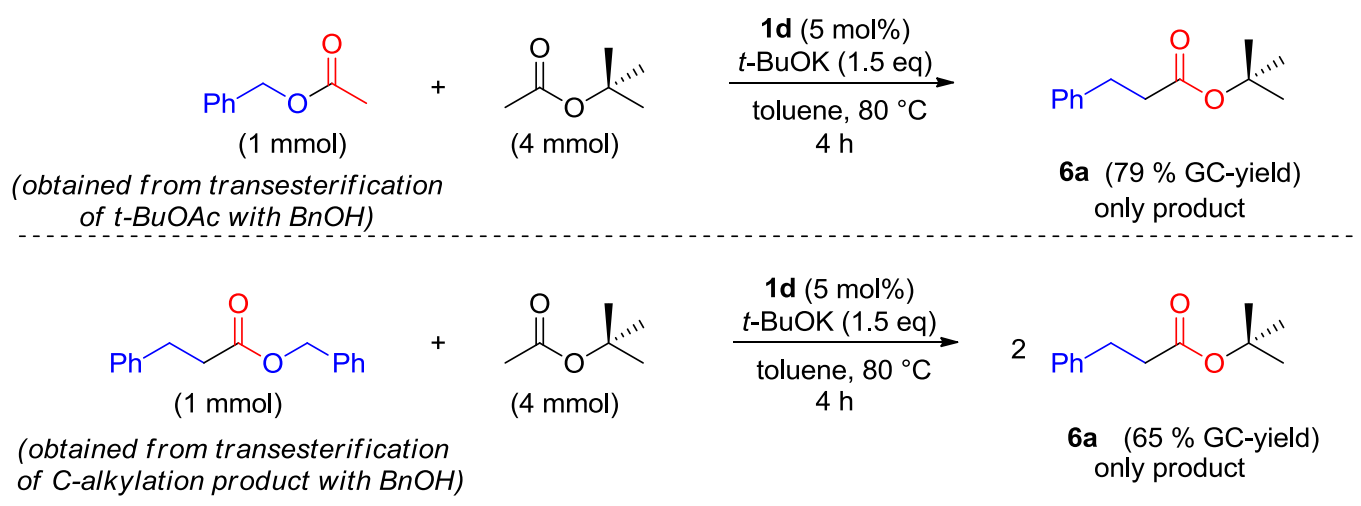

Scheme S 8. Control reactions of transesterification products under catalytic conditions.

Both benzyl acetate and benzyl 3-phenylpropanoate were converted into the alkylated tertbutyl ester 6a under catalytic conditions (Scheme S 8). Conversion was quantitative and no other side-products were observed by GC analysis. 


\section{Characterization data}

\subsection{Amide alkylation products}

$N, N$-dimethyl-3-phenylpropanamide (5a)<smiles>CN(C)CCCc1ccccc1</smiles>

Purification by column chromatography (silica gel, pentane/ethyl acetate, 1:1.5). Yield: 147 mg $(0.830 \mathrm{mmol}, 83 \%)$ colorless oil.

${ }^{1} \mathbf{H}$ NMR $\left(500 \mathrm{MHz}, \mathrm{CDCl}_{3}\right) \delta=7.25$ - 7.31 (m, $\left.2 \mathrm{H}\right), 7.17$ - 7.24 (m, $\left.3 \mathrm{H}\right)$, 3.00-2.94 (m, 8 $\mathrm{H}$, overlapping signals of $\mathrm{NMe}_{2}$ and $\left.\mathrm{CH}_{2}\right), 2.58-2.64(\mathrm{~m}, 2 \mathrm{H}) \mathrm{ppm}$.

${ }^{13}$ C NMR $\left(126 \mathrm{MHz}, \mathrm{CDCl}_{3}\right) \delta=172.3,141.6,128.5,126.2,37.2,35.4,31.5$ ppm.

MS (EI, $70 \mathrm{eV}) \mathrm{m} / z: 177.2\left(\mathrm{M}^{+}\right), 131.1,105.1,91.1,72.1$.

The spectroscopic data correspond to those reported in the literature. ${ }^{4}$

N,N-dimethyl-3-(p-tolyl)propanamide $(\mathbf{5 b})$<smiles>CNC(=O)CCc1ccc(C)cc1</smiles>

Purification by column chromatography (silica gel, pentane/ethyl acetate, 1:1). Yield: $163 \mathrm{mg}$ (0.853 mmol, $85 \%)$ colorless oil.

${ }^{1} \mathbf{H}$ NMR $\left(500 \mathrm{MHz}, \mathrm{CDCl}_{3}\right) \delta=7.05-7.16$ (m, $\left.4 \mathrm{H}\right), 2.87$ - 2.98 (m, $8 \mathrm{H}$, overlapping signals of $\mathrm{NMe}_{2}$ and $\left.\mathrm{CH}_{2}\right), 2.54-2.64(\mathrm{~m}, 2 \mathrm{H}), 2.31$ (s, $\left.3 \mathrm{H}\right) \mathrm{ppm}$.

${ }^{13} \mathrm{C}$ NMR $\left(126 \mathrm{MHz}, \mathrm{CDCl}_{3}\right) \delta=172.3,138.5,135.6,129.2,128.4,37.2,35.6,35.5,31.0$, $21.1 \mathrm{ppm}$.

MS (EI, $70 \mathrm{eV}) \mathrm{m} / z: 191.1\left(\mathrm{M}^{+}\right), 147.1,131.0,118.1,105.1,91.1,72.1$.

The spectroscopic data correspond to those reported in the literature. ${ }^{4}$

N,N-dimethyl-3-(m-tolyl)propanamide (5c)<smiles>CNC(=O)CCc1cccc([N+](=O)[O-])c1</smiles>

Purification by column chromatography (silica gel, pentane/ethyl acetate, 1:1). Yield: $154 \mathrm{mg}$ (0.806 mmol, $81 \%)$ colorless oil.

${ }^{1} \mathbf{H}$ NMR $\left(500 \mathrm{MHz}, \mathrm{CDCl}_{3}\right) \delta=7.13$ - 7.21 (m, $\left.1 \mathrm{H}\right), 6.99$ - 7.07 (m, $\left.3 \mathrm{H}\right), 2.89$ - 2.99 (m, 8 $\mathrm{H}$ overlapping signals of $\mathrm{NMe}_{2}$ and $\left.\mathrm{CH}_{2}\right), 2.56-2.65(\mathrm{~m}, 2 \mathrm{H}), 2.33$ (s, $\left.3 \mathrm{H}\right) \mathrm{ppm}$.

${ }^{13} \mathbf{C}$ NMR $\left(126 \mathrm{MHz}, \mathrm{CDCl}_{3}\right) \delta=172.3,141.5,138.1,129.3,128.5,126.9,125.5,37.2,35.5$, 31.4, $21.5 \mathrm{ppm}$. 
MS (EI, $70 \mathrm{eV}) \mathrm{m} / z: 191.2\left(\mathrm{M}^{+}\right), 147.1,131.1,119.1,105.1,91.1,72.1$.

The spectroscopic data correspond to those reported in the literature. ${ }^{4}$

N,N-dimethyl-3-(o-tolyl)propanamide (5d)<smiles>CC(=O)CCc1ccccc1[N+](=O)[O-]</smiles>

Purification by column chromatography (silica gel, pentane/ethyl acetate, 1:1). Yield: $153 \mathrm{mg}$ (0.801 mmol, $80 \%)$ colorless oil.

${ }^{1} \mathbf{H}$ NMR $\left(500 \mathrm{MHz}, \mathrm{CDCl}_{3}\right) \delta=7.06-7.21$ (m, $\left.5 \mathrm{H}\right), 2.89$ - 3.00 (m, $8 \mathrm{H}$, overlapping signals of $\mathrm{NMe}_{2}$ and $\left.\mathrm{CH}_{2}\right), 2.53-2.60(\mathrm{~m}, 2 \mathrm{H}), 2.33(\mathrm{~s}, 3 \mathrm{H}) \mathrm{ppm}$.

${ }^{13}$ C NMR $\left(126 \mathrm{MHz}, \mathrm{CDCl}_{3}\right) \delta=172.4,140.0,136.1,130.4,128.9,126.4,126.2,37.2,35.5$, 34.0, 28.8, $19.4 \mathrm{ppm}$.

MS (EI, $70 \mathrm{eV}) \mathrm{m} / z: 191.1\left(\mathrm{M}^{+}\right), 176.1,162.1,147.1,119.1,105.1,91.1,72.0$.

The spectroscopic data correspond to those reported in the literature. ${ }^{4}$

N,N-dimethyl-3-(naphthalen-1-yl)propanamide (5e)<smiles>CNC(=O)CCc1cccc2ccccc12</smiles>

Purification by column chromatography (silica gel, pentane/ethyl acetate, 1:1). Yield: $176 \mathrm{mg}$ (0.775 mmol, $78 \%)$ colorless viscous oil.

${ }^{1} \mathbf{H}$ NMR $\left(500 \mathrm{MHz}, \mathrm{CDCl}_{3}\right) \delta=8.03-8.09(\mathrm{~m}, 1 \mathrm{H}), 7.86(\mathrm{dd}, J=8.39,1.07 \mathrm{~Hz}, 1 \mathrm{H}), 7.73$ (d, J=7.63 Hz, $1 \mathrm{H}), 7.45$ - $7.55(\mathrm{~m}, 2 \mathrm{H}), 7.35-7.43(\mathrm{~m}, 2 \mathrm{H}), 3.42-3.48$ (m, $2 \mathrm{H}), 2.96$ (s, 3 $\mathrm{H}), 2.85$ (s, $3 \mathrm{H}), 2.70-2.77(\mathrm{~m}, 2 \mathrm{H}) \mathrm{ppm}$.

${ }^{13} \mathrm{C}$ NMR $\left(126 \mathrm{MHz}, \mathrm{CDCl}_{3}\right) \delta=172.4,137.8,134.0,131.8,129.0,127.1,126.3,126.2$, 125.8, 123.7, 37.2, 35.6, 34.7, $28.6 \mathrm{ppm}$.

MS (EI, $70 \mathrm{eV}) \mathrm{m} / \mathrm{z}: 227.1\left(\mathrm{M}^{+}\right), 183.1,154.1,141.1,128.1,115.1,86.0,72.0$.

HRMS (ESI+) $m / z\left[\mathrm{C}_{15} \mathrm{H}_{17} \mathrm{NO}+\mathrm{H}\right]^{+}$calcd. 228.13829, found 228.13771

The spectroscopic data correspond to those reported in the literature. ${ }^{6}$

3-(2-methoxyphenyl)-N,N-dimethylpropanamide (5f)<smiles>COc1ccccc1CCC(C)=O</smiles>

Purification by column chromatography (silica gel, pentane/ethyl acetate, 1:1.5). Yield: 184 mg (0.889 mmol, $89 \%)$ colorless oil. 
${ }^{1} \mathbf{H}$ NMR $\left(500 \mathrm{MHz}, \mathrm{CDCl}_{3}\right) \delta=7.15-7.22(\mathrm{~m}, 2 \mathrm{H}), 6.87(\mathrm{td}, J=7.40,1.07 \mathrm{~Hz}, 1 \mathrm{H}), 6.84(\mathrm{~d}$, $J=8.24 \mathrm{~Hz}, 1 \mathrm{H}), 3.82(\mathrm{~s}, 3 \mathrm{H}), 2.90-2.97\left(\mathrm{~m}, 8 \mathrm{H}\right.$, overlapping signals of $\mathrm{NMe}_{2}$ and $\mathrm{CH}_{2}$ ), $2.54-2.61(\mathrm{~m}, 2 \mathrm{H}) \mathrm{ppm}$.

${ }^{13} \mathrm{C}$ NMR $\left(126 \mathrm{MHz}, \mathrm{CDCl}_{3}\right) \delta=172.9,157.6,130.3,129.8,127.5,120.6,110.3,55.3,37.2$, 35.4, 33.8, $26.8 \mathrm{ppm}$.

MS (EI, $70 \mathrm{eV}) \mathrm{m} / z: 207.1\left(\mathrm{M}^{+}\right), 192.1,176.1,161.1,150.1,134.1,121.1,105.1,91.1,72.1$, 65.1.

The spectroscopic data correspond to those reported in the literature. ${ }^{4}$

3-(4-methoxyphenyl)-N,N-dimethylpropanamide (5g)<smiles>COc1ccc(CCC(=O)N(C)C)cc1</smiles>

Purification by column chromatography (silica gel, pentane/ethyl acetate, $1: 1 \rightarrow 1: 1.5$ ). Yield: $177 \mathrm{mg}(0.855 \mathrm{mmol}, 86 \%)$ colorless oil.

${ }^{1} \mathbf{H}$ NMR $\left(500 \mathrm{MHz}, \mathrm{CDCl}_{3}\right) \delta=7.07-7.17(\mathrm{~m}, 2 \mathrm{H}), 6.76-6.85(\mathrm{~m}, 2 \mathrm{H}), 3.76(\mathrm{~s}, 3 \mathrm{H})$, 2.91-2.88 (m, $8 \mathrm{H}$, overlapping signals of $\mathrm{NMe}_{2}$ and $\left.\mathrm{CH}_{2}\right), 2.50-2.62(\mathrm{~m}, 2 \mathrm{H}) \mathrm{ppm}$.

${ }^{13} \mathrm{C}$ NMR $\left(126 \mathrm{MHz}, \mathrm{CDCl}_{3}\right) \delta=172.3,158.0,133.6,129.4,113.9,55.3,37.2,36.0,35.4$, $30.5 \mathrm{ppm}$.

MS (EI, $70 \mathrm{eV}) \mathrm{m} / z: 224.1\left(\mathrm{M}^{+}\right), 183.2,154.2,140.1,121.1,100.1,87.1,79.1,72.1,55.1$.

The spectroscopic data correspond to those reported in the literature. ${ }^{4}$

\section{3-(4-chlorophenyl)-N,N-dimethylpropanamide (5h)}<smiles>CNC(=O)CCc1ccc(Cl)cc1</smiles>

Variation from the general procedure: $t$-BuONa $(1.2 \mathrm{mmol})$ was used as base instead of $t$ BuOK. Purification by column chromatography (silica gel, pentane/ethyl acetate, 1:1 $\rightarrow$ 1:2). Yield: $160 \mathrm{mg}(0.758 \mathrm{mmol}, 76 \%)$ colorless oil.

${ }^{1} \mathbf{H}$ NMR $\left(500 \mathrm{MHz}, \mathrm{CDCl}_{3}\right) \delta=7.20$ - 7.25 (m, $\left.2 \mathrm{H}\right), 7.12$ - 7.16 (m, $\left.2 \mathrm{H}\right), 2.89-2.96$ (m, 8 $\mathrm{H}$, overlapping signals of $\mathrm{CH}_{2}$ and $\left.\mathrm{NMe}_{2}\right), 2.54-2.60(\mathrm{~m}, 2 \mathrm{H}) \mathrm{ppm}$.

${ }^{13} \mathbf{C}$ NMR $\left(126 \mathrm{MHz}, \mathrm{CDCl}_{3}\right) \delta=171.9,140.1,131.9,129.9,128.6,37.2,35.5,35.1,30.7$ ppm.

MS (EI, $70 \mathrm{eV}) \mathrm{m} / z: 211.1\left(\mathrm{M}^{+}\right), 167.0,138.0,131.0,125.0,103.0,86.1,72.0,58.0$.

The spectroscopic data correspond to those reported in the literature. ${ }^{4}$ 
N,N-dimethyl-3-(pyridin-4-yl)propanamide (5i)<smiles>CN(C)CCCc1ccncc1</smiles>

Variation from the general procedure: Catalyst $\mathbf{1 f}$ ( $5 \mathrm{~mol} \%$ ) was used. Purification by column chromatography (silica gel, $\left.\mathrm{CH}_{2} \mathrm{Cl}_{2} / \mathrm{MeOH}, 100: 5\right)$. Yield: $125 \mathrm{mg}(0.702 \mathrm{mmol}, 70 \%)$ yellow oil.

${ }^{1} \mathbf{H}$ NMR $\left(500 \mathrm{MHz}, \mathrm{CDCl}_{3}\right) \delta=8.46(\mathrm{~d}, J=4.88 \mathrm{~Hz}, 2 \mathrm{H}), 7.13(\mathrm{~d}, J=5.80 \mathrm{~Hz}, 2 \mathrm{H}), 2.96-$ $2.91\left(\mathrm{~m}, 8 \mathrm{H}\right.$, overlapping signals of $\mathrm{NMe}_{2}$ and $\left.\mathrm{CH}_{2}\right), 2.60(\mathrm{t}, J=7.78 \mathrm{~Hz}, 2 \mathrm{H}) \mathrm{ppm}$.

${ }^{13} \mathbf{C ~ N M R}\left(126 \mathrm{MHz}, \mathrm{CDCl}_{3}\right) \delta=171.3,150.7,149.8,124.0,37.2,35.6,33.8,30.5$ ppm. MS (EI, $70 \mathrm{eV}) \mathrm{m} / z: 178.1\left(\mathrm{M}^{+}\right), 134.4,106.1,72.1$.

HRMS (ESI+) $m / z\left[\mathrm{C}_{10} \mathrm{H}_{14} \mathrm{~N}_{2} \mathrm{O}+\mathrm{H}\right]^{+}$calcd. 179.11789, found 179.11739

N,N-dimethyl-3-(pyridin-3-yl)propanamide (5j)<smiles>CN(C)C(=O)CCc1cccnc1</smiles>

Variation from the general procedure: Catalyst $\mathbf{1 f}$ ( $5 \mathrm{~mol} \%$ ) was used. Purification by column chromatography (silica gel, $\left.\mathrm{CH}_{2} \mathrm{Cl}_{2} / \mathrm{MeOH}, 100: 5\right)$. Yield: $144 \mathrm{mg}$ (0.809 mmol, $81 \%$ ) colorless oil.

${ }^{1}$ H NMR $\left(500 \mathrm{MHz}, \mathrm{CDCl}_{3}\right) \delta=8.46(\mathrm{~s}, 1 \mathrm{H}), 8.42(\mathrm{~d}, J=4.27 \mathrm{~Hz}, 1 \mathrm{H}), 7.54(\mathrm{~d}, J=7.93 \mathrm{~Hz}, 1$ H), $7.18(\mathrm{dd}, J=7.93,4.88 \mathrm{~Hz}, 1 \mathrm{H}), 2.98-2.91\left(\mathrm{~m}, 8 \mathrm{H}\right.$, overlapping signals of $\mathrm{NMe}_{2}$ and $\left.\mathrm{CH}_{2}\right), 2.59(\mathrm{t}, J=7.63 \mathrm{~Hz}, 2 \mathrm{H}) \mathrm{ppm}$.

${ }^{13}$ C NMR $\left(126 \mathrm{MHz}, \mathrm{CDCl}_{3}\right) \delta=171.5,150.0,147.7,136.9,136.2,123.4,37.2,35.6,34.7$, $28.4 \mathrm{ppm}$.

MS (EI, $70 \mathrm{eV}) \mathrm{m} / \mathrm{z}: 177.1\left(\mathrm{M}^{+}\right), 135.1,106.1,92.0,72.0$.

The spectroscopic data correspond to those reported in the literature. ${ }^{5}$

$N, N$-dimethyloctanamide $(\mathbf{5 k})$

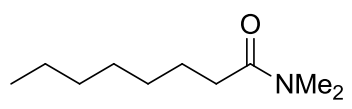

Purification by column chromatography (silica gel, pentane/ethyl acetate, 1:1). Yield: $156 \mathrm{mg}$ (0.912 mmol, $91 \%$ ) colorless oil.

${ }^{1} \mathbf{H}$ NMR $\left(500 \mathrm{MHz}, \mathrm{CDCl}_{3}\right) \delta=3.00(\mathrm{~s}, 3 \mathrm{H}), 2.94(\mathrm{~s}, 3 \mathrm{H}), 2.26-2.34(\mathrm{~m}, 2 \mathrm{H}), 1.57$ - 1.67 (m, $2 \mathrm{H}), 1.20-1.38(\mathrm{~m}, 8 \mathrm{H}), 0.82-0.91(\mathrm{~m}, 3 \mathrm{H}) \mathrm{ppm}$.

${ }^{13}$ C NMR $\left(126 \mathrm{MHz}, \mathrm{CDCl}_{3}\right) \delta=173.4,37.4,35.5,33.6,31.9,29.6,29.3,25.4,22.8,14.2$ ppm.

MS (EI): $m / z$ 171.2 $\left(\mathbf{M}^{+}\right), 142.1,128.1,114.1,100.1,87.1,72.1,57.1$.

The spectroscopic data correspond to those reported in the literature. ${ }^{6}$ 
3-cyclohexyl-N,N-dimethylpropanamide (5l)<smiles>CNC(=O)CCC1CCCCC1</smiles>

Purification by column chromatography (silica gel, pentane/ethyl acetate, 1:1). Yield: $170 \mathrm{mg}$ (0.929 mmol, $93 \%)$ colorless oil.

${ }^{1} \mathbf{H}$ NMR $\left(500 \mathrm{MHz}, \mathrm{CDCl}_{3}\right) \delta=2.98(\mathrm{~s}, 3 \mathrm{H}), 2.91(\mathrm{~s}, 3 \mathrm{H}), 2.25-2.32(\mathrm{~m}, 2 \mathrm{H}), 1.57$ - 1.74 (m, $5 \mathrm{H}), 1.44$ - 1.54 (m, $2 \mathrm{H}), 1.04$ - 1.29 (m, $4 \mathrm{H}), 0.81$ - 0.95 (m, $2 \mathrm{H}) \mathrm{ppm}$.

${ }^{13} \mathrm{C} \mathrm{NMR}\left(126 \mathrm{MHz}, \mathrm{CDCl}_{3}\right) \delta=173.6,37.6,37.4,35.5,33.2,32.7,31.0,26.7,26.4 \mathrm{ppm}$.

MS (EI, $70 \mathrm{eV}): m / z, 183.2\left(\mathrm{M}^{+}\right), 154.2,140.1,121.1,100.1,87.1,72.1,55.1$.

The spectroscopic data correspond to those reported in the literature. ${ }^{4}$

3-cyclopropyl-N,N-dimethylpropanamide (5m)<smiles>CNC(=O)CCC1CC1</smiles>

Purification by column chromatography (silica gel, pentane/ethyl acetate, 1:1). Yield: $114 \mathrm{mg}$ (0.809 mmol, $81 \%)$ colorless oil.

${ }^{1} \mathbf{H}$ NMR $\left(500 \mathrm{MHz}, \mathrm{CDCl}_{3}\right) \delta=3.00(\mathrm{~s}, 3 \mathrm{H}), 2.92(\mathrm{~s}, 3 \mathrm{H}), 2.35-2.44(\mathrm{~m}, 2 \mathrm{H}), 1.48$ - 1.54 (m, $2 \mathrm{H}), 0.66-0.75(\mathrm{~m}, 1 \mathrm{H}), 0.37-0.42(\mathrm{~m}, 2 \mathrm{H}), 0.00-0.05(\mathrm{~m}, 2 \mathrm{H}) \mathrm{ppm}$.

${ }^{13} \mathbf{C ~ N M R}\left(126 \mathrm{MHz}, \mathrm{CDCl}_{3}\right) \delta=173.2,37.4,35.4,33.5,10.8,4.6 \mathrm{ppm}$.

MS (EI, $70 \mathrm{eV}) \mathrm{m} / z$ 141.2 $\left(\mathrm{M}^{+}\right), 126.1,113.1,98.1,87.1,72.1,55.1$.

HRMS (ESI+) $m / z\left[\mathrm{C}_{8} \mathrm{H}_{15} \mathrm{NO}+\mathrm{H}\right]^{+}$calcd. 142.12264, found 142.12217.

$N, N$-dimethyl-5-phenylpentanamide (5n)<smiles>CNC(=O)CCCCc1ccccc1</smiles>

Purification by column chromatography (silica gel, pentane/ethyl acetate, $1: 1 \rightarrow 1: 1.5$ ). Yield: $176 \mathrm{mg}(0.857 \mathrm{mmol}, 86 \%)$ colorless oil.

${ }^{1} \mathbf{H}$ NMR $\left(500 \mathrm{MHz}, \mathrm{CDCl}_{3}\right) \delta=7.24-7.30(\mathrm{~m}, 2 \mathrm{H}), 7.14-7.21(\mathrm{~m}, 3 \mathrm{H}), 2.98(\mathrm{~s}, 3 \mathrm{H}), 2.94$ $(\mathrm{s}, 3 \mathrm{H}), 2.65$ (t, J=7.02 Hz, $2 \mathrm{H}), 2.30-2.36(\mathrm{~m}, 2 \mathrm{H}), 1.63-1.74(\mathrm{~m}, 4 \mathrm{H}) \mathrm{ppm}$.

${ }^{13} \mathbf{C}$ NMR $\left(126 \mathrm{MHz}, \mathrm{CDCl}_{3}\right) \delta=173.0,142.5,128.5,128.4,125.8,37.4,35.9,35.5,33.3$, 31.4, $25.0 \mathrm{ppm}$.

MS (EI, $70 \mathrm{eV}) \mathrm{m} / z: 205.2\left(\mathrm{M}^{+}\right), 117.1,100.1,87.1,72.1$.

The spectroscopic data correspond to those reported in the literature. ${ }^{4}$ 
N-methyl-3-phenylpropanamide (5o)

$\stackrel{\mathrm{Ph}}{\mathrm{NHMe}}_{\mathrm{Ph}}$

Purification by column chromatography (pentane/ethyl acetate, 1:2 $\rightarrow 1: 3$ ). Yield: $89 \mathrm{mg}$ (0.546 mmol, $55 \%)$ crystalline white solid.

${ }^{1} \mathbf{H}$ NMR $\left(500 \mathrm{MHz}, \mathrm{CDCl}_{3}\right) \delta=7.27$ - 7.31 (m, $\left.2 \mathrm{H}\right), 7.17$ - 7.23 (m, $\left.3 \mathrm{H}\right), 5.40$ (br. s., $\left.1 \mathrm{H}\right)$, 2.97 (t, J=7.32 Hz, $2 \mathrm{H}$ ), 2.77 (d, J=4.88 Hz, $3 \mathrm{H}$ ), 2.47 (t, J=7.93 Hz, 2 H) ppm.

${ }^{13}$ C NMR $\left(126 \mathrm{MHz}, \mathrm{CDCl}_{3}\right) \delta=172.8,141.1,128.7,128.5,126.4,38.6,31.9,26.4$ ppm.

MS (EI, $70 \mathrm{eV}) \mathrm{m} / z: 163.1\left(\mathrm{M}^{+}\right), 133.1,105.1,91.1,77.1,65.1,58.1,51.1$.

The spectroscopic data correspond to those reported in the literature. ${ }^{4}$

\section{$N, N$-diethyl-3-phenylpropanamide (5p)}<smiles>CCN(CC)C(=O)CCc1ccccc1</smiles>

Variation from the general procedure: $5 \mathrm{~mol} \%$ of catalyst 1d was used. Purification by column chromatography (pentane/ethyl acetate, 1:1.5). Yield: $156 \mathrm{mg}(0.761 \mathrm{mmol}, 76 \%)$ colorless oil.

${ }^{1} \mathbf{H}$ NMR $\left(500 \mathrm{MHz}, \mathrm{CDCl}_{3}\right) \delta=7.15-7.32(\mathrm{~m}, 5 \mathrm{H}), 3.38(\mathrm{q}, J=7.12 \mathrm{~Hz}, 2 \mathrm{H}), 3.22$ (q, $J=7.02 \mathrm{~Hz}, 2 \mathrm{H}), 2.96-3.01(\mathrm{~m}, 2 \mathrm{H}), 2.56-2.62$ (m, $2 \mathrm{H}), 1.10$ (td, J=7.17, $4.58 \mathrm{~Hz}, 6 \mathrm{H})$ ppm.

${ }^{13} \mathbf{C}$ NMR $\left(126 \mathrm{MHz}, \mathrm{CDCl}_{3}\right) \delta=171.4,141.8,128.60,128.59,126.2,42.0,40.3,35.3,31.8$, 14.4, $13.2 \mathrm{ppm}$.

MS (EI, $70 \mathrm{eV}) \mathrm{m} / \mathrm{z}: 205.2\left(\mathrm{M}^{+}\right), 176.1,133.1,120.1,105.1,91.1,72.1,58.1$.

The spectroscopic data correspond to those reported in the literature. ${ }^{4}$

3-phenyl-1-(pyrrolidin-1-yl)propan-1-one (5q)<smiles>O=C(CCc1ccccc1)N1CCCC1</smiles>

Purification by column chromatography (pentane/ethyl acetate, 1:2). Yield: $133 \mathrm{mg}$ (0.655 mmol, $66 \%$ ) colorless oil.

${ }^{1} \mathbf{H}$ NMR $\left(500 \mathrm{MHz}, \mathrm{CDCl}_{3}\right) \delta=7.27-7.31(\mathrm{~m}, 2 \mathrm{H}), 7.17-7.25(\mathrm{~m}, 3 \mathrm{H}), 3.47(\mathrm{t}, J=6.87$ $\mathrm{Hz}, 2 \mathrm{H}), 3.29$ (t, J=6.71 Hz, 2 H), $2.96-3.02$ (m, 2 H), 2.54 - 2.59 (m, 2 H), 1.85 - 1.92 (m, 2 $\mathrm{H}), 1.79-1.85(\mathrm{~m}, 2 \mathrm{H}) \mathrm{ppm}$.

${ }^{13}$ C NMR $\left(126 \mathrm{MHz}, \mathrm{CDCl}_{3}\right) \delta=170.9,141.6,128.5,126.1,46.6,45.7,36.8,31.3,26.1,24.4$ ppm.

MS (EI, $70 \mathrm{eV}) \mathrm{m} / \mathrm{z}: 203.2\left(\mathrm{M}^{+}\right), 112.1,91.1,70.1,55.1$.

The spectroscopic data correspond to those reported in the literature. ${ }^{7}$ 
3-phenyl-1-(piperidin-1-yl)propan-1-one (5r)<smiles>O=C(CCc1ccccc1)N1CCCCC1</smiles>

Purification by column chromatography (pentane/ethyl acetate, 1:1). Yield: $167 \mathrm{mg}(0.770$ mmol, $77 \%$ ) colorless oil.

${ }^{1} \mathbf{H}$ NMR $\left(500 \mathrm{MHz}, \mathrm{CDCl}_{3}\right) \delta=7.27-7.34(\mathrm{~m}, 2 \mathrm{H}), 7.18-7.27$ (m, $\left.3 \mathrm{H}\right), 3.57$ (t, J=5.80 Hz, 2 H), 3.34 (t, J=5.49 Hz, 2 H), 2.98 (t, J=7.63 Hz, 2 H), 2.63 (t, J=8.24 Hz, 2 H), 1.59 1.66 (m, $2 \mathrm{H}), 1.50$ - 1.58 (m, $2 \mathrm{H}), 1.43-1.50$ (m, $2 \mathrm{H}) \mathrm{ppm}$.

${ }^{13}$ C NMR $\left(126 \mathrm{MHz} \mathrm{CDCl}_{3}\right) \delta=170.5,141.5,128.53,128.50,126.1,46.7,42.8,35.3,31.7$, 26.4, 25.6, $24.6 \mathrm{ppm}$.

MS (EI, $70 \mathrm{eV}) \mathrm{m} / \mathrm{z}: 217.2\left(\mathrm{M}^{+}\right), 126.1,112.1,105.1,91.1,84.1,77.1,69.1,56.1$.

The spectroscopic data correspond to those reported in the literature. ${ }^{4}$

1-morpholino-3-phenylpropan-1-one (5s)<smiles>O=C(CCc1ccccc1)N1CCOCC1</smiles>

Purification by column chromatography (silica gel, pentane/ethyl acetate, $1: 1 \rightarrow 1: 3$ ). Yield: $183 \mathrm{mg}$ (0.836 mmol, $84 \%)$ colorless oil which solidified upon standing (pale yellow solid).

${ }^{1} \mathbf{H}$ NMR $\left(500 \mathrm{MHz}, \mathrm{CDCl}_{3}\right) \delta=7.25$ - $7.31(\mathrm{~m}, 2 \mathrm{H}), 7.17$ - $7.22(\mathrm{~m}, 3 \mathrm{H}), 3.57$ - $3.64(\mathrm{~m}, 4$ H), 3.47 - 3.53 (m, $2 \mathrm{H}), 3.31$ - 3.38 (m, $2 \mathrm{H}), 2.94$ - 3.00 (m, $2 \mathrm{H}), 2.57$ - 2.64 (m, $2 \mathrm{H}) \mathrm{ppm}$.

${ }^{13}$ C NMR $\left(126 \mathrm{MHz}, \mathrm{CDCl}_{3}\right) \delta=170.9,141.1,128.6,128.5,126.4,126.3,67.0,66.5,46.0$, 42.0, 34.9, $31.5 \mathrm{ppm}$.

MS (EI, $70 \mathrm{eV}) \mathrm{m} / \mathrm{z}: 219.2\left(\mathrm{M}^{+}\right), 128.1,105.1,91.1,77.1,57.1$.

The spectroscopic data correspond to those reported in the literature. ${ }^{4}$

3-(4-methoxyphenyl)-1-morpholinopropan-1-one (5t)<smiles>COc1ccc(CCC(=O)N2CCOCC2)cc1</smiles>

Representative procedure for $10 \mathrm{mmol}$ scale reaction: Using a glove box, a pressure tube (Ace pressure tube, $38 \mathrm{~mL}$ volume) was charged with a magnetic stirring bar, 4-acetyl morpholine (20 mmol, $2.30 \mathrm{~mL}, 2.0 \mathrm{eq}), t$-BuOK (12.0 mmol, $1.34 \mathrm{~g}, 1.2 \mathrm{eq})$, 4methoxybenzyl alcohol (10 mmol, $1.24 \mathrm{~mL}, 1.0 \mathrm{eq})$, catalyst $1 \mathrm{~d}(0.250 \mathrm{mmol}, 132 \mathrm{mg}, 2.5$ mol\%) and THF (10 mL). The tube was sealed with a Teflon cap and immersed into a $100{ }^{\circ} \mathrm{C}$ oil bath outside the glove box for 24 hours. After cooling, the reaction mixture was quenched with half-saturated aqueous $\mathrm{NaCl}$ solution $(10 \mathrm{~mL})$ and extracted with diethyl ether $(3 \mathrm{x} 100$ $\mathrm{mL}$ ). The combined organic phase was dried over $\mathrm{Na}_{2} \mathrm{SO}_{4}$ and concentrated. The crude product was nearby pure as judged by ${ }^{1} \mathrm{H}$ NMR analysis and was purified using a small pad (5 
$\mathrm{cm}$ height) of silica gel eluting with pentane/ethyl acetate, 1:2 to give the product as a colorless oil (2.01 g, $8.06 \mathrm{mmol}, 81 \%)$.

The reaction gave the same yields on a 1 and $5 \mathrm{mmol}$ scale.

${ }^{1} \mathbf{H}$ NMR $\left(500 \mathrm{MHz}, \mathrm{CDCl}_{3}\right) \delta=7.08-7.17(\mathrm{~m}, 2 \mathrm{H}), 6.79-6.87(\mathrm{~m}, 2 \mathrm{H}), 3.78(\mathrm{~s}, 3 \mathrm{H}), 3.58$ - 3.67 (m, 4 H), 3.50 - 3.57 (m, 2 H), 3.31 - 3.40 (m, 2 H), 2.87 - 2.95 (m, 2 H), 2.53 - 2.62 (m, $2 \mathrm{H}) \mathrm{ppm}$.

${ }^{13}$ C NMR $\left(126 \mathrm{MHz}, \mathrm{CDCl}_{3}\right) \delta=171.1,158.2,133.2,129.5,114.1,67.0,66.6,55.4,46.1$, 42.0, 35.2, $30.7 \mathrm{ppm}$.

MS (EI, $70 \mathrm{eV}) \mathrm{m} / z: 249.2\left(\mathrm{M}^{+}\right), 161.1,134.1,121.1,108.1,86.1,70.1,57.1$.

The spectroscopic data correspond to those reported in the literature. ${ }^{7}$

1-morpholino-5-phenylpentan-1-one (5u)<smiles>O=C(CCCCc1ccccc1)N1CCOCC1</smiles>

$10 \mathrm{mmol}$ scale. Purification by column chromatography (pentane/ethyl acetate, 6:4 $\rightarrow 1: 1$ ). Yield: $2.02 \mathrm{~g}(8.17 \mathrm{mmol}, 82 \%)$ colorless oil.

${ }^{1} \mathbf{H}$ NMR $\left(500 \mathrm{MHz}, \mathrm{CDCl}_{3}\right) \delta=7.21-7.25(\mathrm{~m}, 2 \mathrm{H}), 7.12-7.19(\mathrm{~m}, 3 \mathrm{H}), 3.55-3.66(\mathrm{~m}, 6$ H), 3.35 - 3.42 (m, 2 H), 2.58 - 2.67 (m, 2 H), 2.24 - 2.34 (m, 2 H), 1.60 - 1.71 (m, 4 H) ppm.

${ }^{13} \mathbf{C ~ N M R}\left(126 \mathrm{MHz}, \mathrm{CDCl}_{3}\right) \delta=171.7,142.3,128.5,125.9,67.1,66.8,46.1,42.0,35.8,33.1$, 31.2, $25.0 \mathrm{ppm}$.

MS (EI, $70 \mathrm{eV}) \mathrm{m} / z: 247.2\left(\mathrm{M}^{+}\right), 156.1,142.1,129.1,117.1,104.1,91.1,70.1,57.2$.

The spectroscopic data correspond to those reported in the literature. ${ }^{8}$

3-cyclopropyl-1-morpholinopropan-1-one (5v)<smiles>O=C(CCC1CC1)N1CCOCC1</smiles>

5 mmol scale. Purification by column chromatography (pentane/ethyl acetate, 1:1). Yield: 780 $\mathrm{mg}(4.26 \mathrm{mmol}, 85 \%)$ colorless oil.

${ }^{1} \mathbf{H}$ NMR $\left(500 \mathrm{MHz}, \mathrm{CDCl}_{3}\right) \delta=3.64$ - $3.70(\mathrm{~m}, 1 \mathrm{H}), 3.58-3.63(\mathrm{~m}, 1 \mathrm{H}), 3.46-3.52(\mathrm{~m}, 1$ H), 2.38 - $2.45(\mathrm{~m}, 1 \mathrm{H}), 1.49-1.57(\mathrm{~m}, 1 \mathrm{H}), 0.67$ - $0.77(\mathrm{~m}, 1 \mathrm{H}), 0.41-0.46(\mathrm{~m}, 1 \mathrm{H}), 0.03$ - 0.08 (m, $2 \mathrm{H}) \mathrm{ppm}$.

${ }^{13}$ C NMR $\left(126 \mathrm{MHz}, \mathrm{CDCl}_{3}\right) \delta=171.9,67.1,66.8,46.2,42.0,33.2,30.6,10.8,4.7$ ppm. MS (EI, $70 \mathrm{eV}) \mathrm{m} / z: 183.1\left(\mathrm{M}^{+}\right), 168.1,154.1,140.1,129.1,114.1,97.1,86.1,70.1,57.1$. HRMS (ESI+): $m / z\left[\mathrm{C}_{10} \mathrm{H}_{17} \mathrm{NO}_{2}+\mathrm{H}\right]^{+}$calcd. 184.13321, found 184.13268. 


\subsection{Ester alkylation products}

tert-butyl 3-phenylpropanoate (6a)<smiles>CC(C)(C)OC(=O)CCc1ccccc1</smiles>

Purification by column chromatography (silica gel, pentane/diethyl ether, 50:1). Yield: 145 mg (0.704 mmol, $70 \%)$ colorless oil.

${ }^{1} \mathbf{H}$ NMR $\left(500 \mathrm{MHz}, \mathrm{CDCl}_{3}\right) \delta=7.16-7.23(\mathrm{~m}, 2 \mathrm{H}), 7.08-7.15$ (m, $\left.3 \mathrm{H}\right), 2.83$ (t, J=7.78 $\mathrm{Hz}, 2 \mathrm{H}), 2.43-2.49$ (m, $2 \mathrm{H}), 1.34$ (s, $9 \mathrm{H}) \mathrm{ppm}$.

${ }^{13}$ C NMR $\left(126 \mathrm{MHz}, \mathrm{CDCl}_{3}\right) \delta=172.4,140.9,128.50,128.45,126.2,80.4,37.2,31.3,28.2$ ppm.

MS (EI, $70 \mathrm{eV}) \mathrm{m} / z: 206.1\left(\mathrm{M}^{+}\right), 150.1,133.1,104.1,91.1,77.1,57.1$.

The spectroscopic data correspond to those reported in the literature. ${ }^{9}$

tert-butyl 3-(p-tolyl)propanoate $(\boldsymbol{6} \boldsymbol{b})$<smiles>Cc1ccc(CCC(=O)OC(C)(C)C)cc1</smiles>

Purification by column chromatography (silica gel, pentane/diethyl ether, 50:1). Yield: 149 mg $(0.677 \mathrm{mmol}, 68 \%)$ colorless oil.

${ }^{1} \mathbf{H}$ NMR $\left(500 \mathrm{MHz}, \mathrm{CDCl}_{3}\right) \delta=7.11(\mathrm{~s}, 4 \mathrm{H}), 2.89(\mathrm{t}, J=7.63 \mathrm{~Hz}, 2 \mathrm{H}), 2.53(\mathrm{t}, J=8.24 \mathrm{~Hz}, 2$ $\mathrm{H}), 2.33$ (s, $3 \mathrm{H}), 1.44$ (s, $9 \mathrm{H}) \mathrm{ppm}$.

${ }^{13}$ C NMR $\left(126 \mathrm{MHz}, \mathrm{CDCl}_{3}\right) \delta=172.5,137.8,135.6,129.2,128.3,80.4,37.4,30.8,28.2$, $21.1 \mathrm{ppm}$.

MS (EI, $70 \mathrm{eV}) m / z: 220.2\left(\mathrm{M}^{+}\right), 164.1,147.1,118.1,105.1,91.1,77.1,65.1,57.1$

The spectroscopic data correspond to those reported in the literature. ${ }^{9}$

tert-butyl 3-(m-tolyl)propanoate (6c)<smiles>Cc1cccc(CCC(=O)OC(C)(C)C)c1</smiles>

Purification by column chromatography (silica gel, pentane/diethyl ether, 50:1). Yield: 168 mg $(0.764 \mathrm{mmol}, 76 \%)$ colorless oil.

${ }^{1} \mathbf{H}$ NMR $\left(500 \mathrm{MHz}, \mathrm{CDCl}_{3}\right) \delta=7.19(\mathrm{t}, \mathrm{J}=7.48 \mathrm{~Hz}, 1 \mathrm{H}), 6.99-7.06(\mathrm{~m}, 3 \mathrm{H}), 2.90$ (t, $J=7.93 \mathrm{~Hz}, 2 \mathrm{H}), 2.52-2.58$ (m, $2 \mathrm{H}), 2.34$ (s, $3 \mathrm{H}), 1.45$ (s, $9 \mathrm{H}) \mathrm{ppm}$.

${ }^{13}$ C NMR $\left(126 \mathrm{MHz}, \mathrm{CDCl}_{3}\right) \delta=172.4,140.8,138.0,129.3,128.4,126.9,125.4,80.4,37.2$, 31.2, 28.2, $21.5 \mathrm{ppm}$.

MS (EI, $70 \mathrm{eV}) \mathrm{m} / z: 220.2\left(\mathrm{M}^{+}\right), 164.1,147.1,118.1,105.1,91.1,77.1,65.1,57.2$. 
The spectroscopic data correspond to those reported in the literature. ${ }^{9}$

tert-butyl 3-(o-tolyl)propanoate $(\mathbf{6 d})$<smiles>CC(C)(C)OC(=O)CCc1ccccc1[N+](=O)[O-]</smiles>

Purification by column chromatography (silica gel, pentane/diethyl ether, 50:1). Yield: 138 $\mathrm{mg}(0.626 \mathrm{mmol}, 63 \%)$ colorless oil.

${ }^{1} \mathbf{H}$ NMR $\left(500 \mathrm{MHz}, \mathrm{CDCl}_{3}\right) \delta=7.06-7.19(\mathrm{~m}, 4 \mathrm{H}), 2.86-2.96(\mathrm{~m}, 3 \mathrm{H}), 2.48-2.55(\mathrm{~m}, 2$ H), 2.34 (s, $3 \mathrm{H}), 1.45$ (s, $9 \mathrm{H}) \mathrm{ppm}$.

${ }^{13}$ C NMR $\left(126 \mathrm{MHz}, \mathrm{CDCl}_{3}\right) \delta=172.56,139.0,136.1,130.3,128.6,126.4,126.1,80.5,35.9$, 28.6, 28.2, $19.4 \mathrm{ppm}$.

MS (EI, $70 \mathrm{eV}) \mathrm{m} / z: 220.2\left(\mathrm{M}^{+}\right), 164.1,147.1,119.1,105.1,91.1,77.1,65.1,57.1$.

The spectroscopic data correspond to those reported in the literature. ${ }^{9}$

tert-butyl 3-(naphthalen-1-yl)propanoate (6e)<smiles>CC(C)(C)OC(=O)CCc1cccc2ccccc12</smiles>

Purification by column chromatography (silica gel, pentane/diethyl ether, 50:1). Yield: 161 $\mathrm{mg}(0.629 \mathrm{mmol}, 63 \%)$ colorless oil.

${ }^{1} \mathbf{H}$ NMR $\left(500 \mathrm{MHz}, \mathrm{CDCl}_{3}\right) \delta=8.06(\mathrm{~d}, \mathrm{~J}=8.54 \mathrm{~Hz}, 1 \mathrm{H}), 7.87(\mathrm{~d}, \mathrm{~J}=7.93 \mathrm{~Hz}, 1 \mathrm{H}), 7.74(\mathrm{~d}$, $\mathrm{J}=7.93 \mathrm{~Hz}, 1 \mathrm{H}), 7.46-7.57$ (m, $2 \mathrm{H}), 7.34$ - 7.44 (m, $2 \mathrm{H}), 3.35$ - 3.44 (m, $2 \mathrm{H}), 2.65$ - 2.73 (m, $2 \mathrm{H}), 1.42-1.51(\mathrm{~m}, 9 \mathrm{H}) \mathrm{ppm}$.

${ }^{13}$ C NMR $\left(126 \mathrm{MHz}, \mathrm{CDCl}_{3}\right) \delta=172.5,136.9,134.0,131.8,128.9,127.1,126.1,126.0$, $125.7,123.6,80.6,36.5,28.4,28.2 \mathrm{ppm}$.

MS (EI, $70 \mathrm{eV}) \mathrm{m} / z: 256.1\left(\mathrm{M}^{+}\right), 200.1,183.1,153.1,141.1,128.1,115.0,77.0,57.1$.

Elemental analysis (\%) for $\mathrm{C}_{17} \mathrm{H}_{20} \mathrm{O}_{2}$ calcd. C 79.65, H 7.86; found: $\mathrm{C} 79.55, \mathrm{H}$ 7.68.

tert-butyl 3-(4-methoxyphenyl)propanoate (6f)<smiles>COc1ccc(CCC(=O)OC(C)(C)C)cc1</smiles>

Purification by column chromatography (silica gel, pentane/diethyl ether, 30:1). Yield: 181 $\mathrm{mg}(0.767 \mathrm{mmol}, 77 \%)$ colorless oil.

${ }^{1} \mathbf{H}$ NMR $\left(300 \mathrm{MHz}, \mathrm{CDCl}_{3}\right) \delta=7.12(\mathrm{~d}, J=8.20 \mathrm{~Hz}, 2 \mathrm{H}), 6.82(\mathrm{~d}, J=8.20 \mathrm{~Hz}, 2 \mathrm{H}), 3.78(\mathrm{~s}, 3$ H), $2.85(\mathrm{t}, J=8.20 \mathrm{~Hz}, 2 \mathrm{H}), 2.50(\mathrm{t}, J=7.61 \mathrm{~Hz}, 2 \mathrm{H}), 1.42(\mathrm{~s}, 9 \mathrm{H}) \mathrm{ppm}$.

${ }^{13}$ C NMR $\left(75 \mathrm{MHz}, \mathrm{CDCl}_{3}\right) \delta=172.5,133.0,129.4,113.9,80.4,55.4,37.5,30.4,28.2$ ppm. 
MS (EI, $70 \mathrm{eV}) \mathrm{m} / z: 236.1\left(\mathrm{M}^{+}\right), 180.1,163.1,137.0,121.1,91.1,77.0,57.1$.

The spectroscopic data correspond to those reported in the literature. ${ }^{10}$

tert-butyl 3-(2-methoxyphenyl)propanoate (6g)<smiles>COc1ccccc1CCC(=O)OC(C)(C)C</smiles>

Purification by column chromatography (silica gel, pentane/diethyl ether, 40:1). Yield: 165 mg (0.699 mmol, $70 \%)$ colorless oil.

${ }^{1}$ H NMR $\left(500 \mathrm{MHz}, \mathrm{CDCl}_{3}\right) \delta=7.10$ - 7.24 (m, $\left.2 \mathrm{H}\right), 6.78$ - 6.93 (m, $\left.2 \mathrm{H}\right), 3.83$ (s, $\left.3 \mathrm{H}\right), 2.84$ - 2.96 (m, $2 \mathrm{H}), 2.46-2.58$ (m, $2 \mathrm{H}), 1.43$ (s, $9 \mathrm{H}) \mathrm{ppm}$.

${ }^{13} \mathbf{C}$ NMR $\left(126 \mathrm{MHz}, \mathrm{CDCl}_{3}\right) \delta=172.9,157.6,130.0,129.2,127.5,120.4,110.3,80.1,55.3$, $35.5,28.2,26.3 \mathrm{ppm}$.

MS (EI, $70 \mathrm{eV}) \mathrm{m} / z: 236.1\left(\mathrm{M}^{+}\right), 180.1,163.0,134.1,121.1,105.1,91.1,77.0,65.1,57.1$.

Elemental analysis $(\%)$ for $\mathrm{C}_{14} \mathrm{H}_{20} \mathrm{O}_{3}$ calcd. C 71.16, H 8.53; found: C 71.08, H 8.82.

Due to low polarity of the compound, no ESI+ HRMS could be obtained.

tert-butyl 3-(furan-2-yl)propanoate (6h)<smiles>CC(C)OC(=O)CCc1ccco1</smiles>

Purification by column chromatography (silica gel, pentane/diethyl ether, 50:1). Yield: 107 mg $(0.546 \mathrm{mmol}, 55 \%)$ colorless oil.

${ }^{1}$ H NMR $\left(500 \mathrm{MHz}, \mathrm{CDCl}_{3}\right) \delta=7.29(\mathrm{dd}, J=1.83,0.92 \mathrm{~Hz}, 1 \mathrm{H}), 6.27(\mathrm{dd}, J=3.05,1.83 \mathrm{~Hz}, 1$ H), $6.00(\mathrm{dd}, J=3.36,0.92 \mathrm{~Hz}, 1 \mathrm{H}), 2.92$ (t, J=7.63 Hz, $1 \mathrm{H}), 2.53-2.59$ (m, $2 \mathrm{H}), 1.43$ (s, 9 H) ppm.

${ }^{13}$ C NMR $\left(126 \mathrm{MHz}, \mathrm{CDCl}_{3}\right) \delta=172.0,154.6,141.2,110.3,105.3,80.6,34.0,28.2,23.8$ ppm.

MS (EI, $70 \mathrm{eV}) \mathrm{m} / z: 196.1\left(\mathrm{M}^{+}\right), 140.0,123.0,94.0,81.0,65.1,57.1$.

The spectroscopic data correspond to those reported in the literature. ${ }^{9}$

tert-butyl 3-(pyridin-3-yl)propanoate (6i)<smiles>CC(C)(C)OC(=O)CCc1cccnc1</smiles>

Purification by column chromatography (silica gel, pentane/diethyl ether, 50:1). Yield: 100 mg (0.483 mmol, $48 \%)$ colorless oil.

${ }^{1} \mathbf{H}$ NMR $\left(500 \mathrm{MHz}, \mathrm{CDCl}^{3}\right) \delta=8.35-8.53(\mathrm{~m}, 2 \mathrm{H}), 7.50(\mathrm{dt}, J=7.63,1.83 \mathrm{~Hz}, 1 \mathrm{H}), 7.18$ (dd, $J=7.78,4.73 \mathrm{~Hz}, 1 \mathrm{H}), 2.88$ (t, J=7.63 Hz, $2 \mathrm{H}$ ), 2.53 (t, J=7.48 Hz, 2 H), 1.38 (s, $9 \mathrm{H}$ ) ppm. 
${ }^{13}$ C NMR $\left(126 \mathrm{MHz}, \mathrm{CDCl}_{3}\right) \delta=171.8,150.0,147.8,136.1,136.0,123.4,80.8,36.6,28.3$, $28.1 \mathrm{ppm}$.

MS (EI, $70 \mathrm{eV}) \mathrm{m} / z: 207.1\left(\mathrm{M}^{+}\right), 192.1,162.1,151.0,134.0,106.1,92.0,78.0,65.1,57.1$.

The spectroscopic data correspond to those reported in the literature. ${ }^{11}$

tert-butyl 3-(4-chlorophenyl)propanoate (6j)<smiles>CC(C)(C)OC(=O)CCc1ccc(Cl)cc1</smiles>

Variation from the general procedure: $t$ - $\mathrm{BuONa}$ (1.5 equiv) was used as the base. Purification by column chromatography (pentane/diethyl ether, 50:1). Yield: $174 \mathrm{mg}(0.723 \mathrm{mmol}, 72 \%)$ colorless oil.

${ }^{1} \mathbf{H}$ NMR $\left(300 \mathrm{MHz}, \mathrm{CDCl}_{3}\right) \delta=7.21-7.31(\mathrm{~m}, 2 \mathrm{H}), 7.08-7.20(\mathrm{~m}, 2 \mathrm{H}), 2.78-2.96(\mathrm{~m}, 2$ $\mathrm{H}), 2.46-2.62(\mathrm{~m}, 2 \mathrm{H}), 1.43(\mathrm{~s}, 9 \mathrm{H}) \mathrm{ppm}$.

${ }^{13} \mathrm{C}$ NMR $\left(126 \mathrm{MHz}, \mathrm{CDCl}_{3}\right) \delta=172.1,139.4,132.0,129.8,128.6,80.6,37.0,30.6,28.2$ ppm.

MS (EI, $70 \mathrm{eV}) \mathrm{m} / z: 240.1\left(\mathrm{M}^{+}\right), 225.0,207.0,184.0,167.0,149.0,138.0,125.0,112.0$, 103.1, 89.0, 77.0, 57.1

The spectroscopic data correspond to those reported in the literature. ${ }^{9}$

tert-butyl 3-(4-fluorophenyl)propanoate $(\boldsymbol{6} \boldsymbol{k})$<smiles>CC(C)(C)OC(=O)CCc1ccc(F)cc1</smiles>

$2 \mathrm{~mL}$ toluene were used. Purification by column chromatography (silica gel, pentane/diethyl ether, 50:1). Yield: $184 \mathrm{mg}(0.821 \mathrm{mmol}, 82 \%)$ colorless oil.

${ }^{1} \mathbf{H}$ NMR $\left(500 \mathrm{MHz}, \mathrm{CDCl}_{3}\right) \delta=7.15(\mathrm{dd}, J=8.39,5.34 \mathrm{~Hz}, 2 \mathrm{H}), 6.96(\mathrm{t}, J=8.70 \mathrm{~Hz}, 2 \mathrm{H})$, $2.88(\mathrm{t}, J=7.63 \mathrm{~Hz}, 2 \mathrm{H}), 2.51(\mathrm{t}, J=7.78 \mathrm{~Hz}, 2 \mathrm{H}), 1.41(\mathrm{~s}, 9 \mathrm{H}) \mathrm{ppm}$.

${ }^{13}$ C NMR (126 MHz, $\left.\mathrm{CDCl}_{3}\right) \delta=172.2,162.5,160.6,136.54^{*}, 136.51^{*}, 129.9^{*}, 129.8^{*}$, $115.3^{*}, 115.2^{*}, 80.6,37.3,30.5,28.2 \mathrm{ppm}$.

${ }^{*}{ }^{13} \mathrm{C}-{ }^{19} \mathrm{~F}$ coupling is observed.

MS (EI, $70 \mathrm{eV}) \mathrm{m} / z: 224.1\left(\mathrm{M}^{+}\right), 168.1,151.1,122.1,109.1,103.1,96.1,83.1,77.1,57.1$.

The spectroscopic data correspond to those reported in the literature. ${ }^{9}$

tert-butyl 5-phenylpentanoate (6l)<smiles>CC(C)(C)OC(=O)CCCCc1ccccc1</smiles>

Purification by column chromatography (silica gel, pentane/diethyl ether, 50:1). Yield: 135 $\mathrm{mg}(0.576 \mathrm{mmol}, 58 \%)$ colorless oil. 
When conducted at $100{ }^{\circ} \mathrm{C}$ (oil bath), the reaction gave the same isolated yield.

${ }^{1} \mathbf{H}$ NMR $\left(500 \mathrm{MHz}, \mathrm{CDCl}_{3}\right) \delta=7.15$ - $7.25(\mathrm{~m}, 10 \mathrm{H}), 7.05-7.14(\mathrm{~m}, 3 \mathrm{H}), 2.50-2.59$ (m, 2 H), 2.12 - $2.20(\mathrm{~m}, 2 \mathrm{H}), 1.56(\mathrm{dt}, J=7.32,3.66 \mathrm{~Hz}, 4 \mathrm{H}), 1.36(\mathrm{~s}, 9 \mathrm{H}) \mathrm{ppm}$.

${ }^{13} \mathrm{C}$ NMR $\left(126 \mathrm{MHz}, \mathrm{CDCl}_{3}\right) \delta=173.2,142.2,128.5,128.4,125.9,80.1,35.8,35.5,31.0$, 28.2, $24.9 \mathrm{ppm}$.

MS (EI, $70 \mathrm{eV}) \mathrm{m} / z: 234.1\left(\mathrm{M}^{+}\right), 178.1,161.1,117.1,104.1,91.1,77.1,65.0,57.1$.

The spectroscopic data correspond to those reported in the literature. ${ }^{9}$

\subsection{Follow-up products}

1-(4-methoxyphenyl)heptan-3-one (7a)

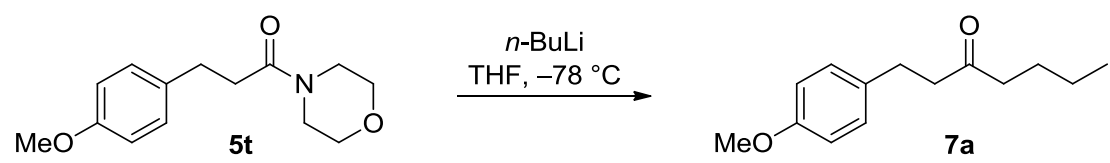

To a solution of amide $5 \mathrm{t}$ ( $255 \mathrm{mg}, 1.02 \mathrm{mmol}, 1 \mathrm{eq})$ in abs. THF (5 mL) was added $n$-BuLi (1.6 $\mathrm{M}$ in hexane, $3 \mathrm{mmol}, 3$ equiv, $1.88 \mathrm{~mL}$ ) dropwise at $-78{ }^{\circ} \mathrm{C}$. After $3 \mathrm{~h}$ and $3 \mathrm{~h} 40 \mathrm{~min}$ aliquots of $n$-BuLi $(0.5 \mathrm{mmol}$ and $0.3 \mathrm{mmol}$, respectively) were added in addition. After $4 \mathrm{~h}$ 20 min overall reaction time the reaction was quenched with aqueous acetic acid $(30 \%, 5$ $\mathrm{mL})$, warmed to $\mathrm{rt}$ and extracted with diethyl ether $(3 \times 30 \mathrm{~mL})$. The combined organic phase was dried over $\mathrm{Na}_{2} \mathrm{SO}_{4}$ and concentrated. Purification by column chromatography (silica gel, pentane/diethyl ether, 6:1) gave ketone 7a as a colorless oil ( $207 \mathrm{mg}, 0.941 \mathrm{mmol}, 92 \%$ ).

${ }^{1} \mathbf{H}$ NMR $\left(300 \mathrm{MHz}, \mathrm{CDCl}_{3}\right) \delta=7.03-7.15$ (m, $\left.2 \mathrm{H}\right), 6.76-6.89(\mathrm{~m}, 2 \mathrm{H}), 3.78(\mathrm{~s}, 3 \mathrm{H}), 2.78$ - 2.90 (m, 2 H), 2.63 - 2.76 (m, 2 H), 2.37 (t, J=7.32 Hz, 2 H), 1.44 - 1.61 (m, 2 H), 1.28 (dq, $J=14.86,7.35 \mathrm{~Hz}, 2 \mathrm{H}), 0.88(\mathrm{t}, J=7.32 \mathrm{~Hz}, 3 \mathrm{H}) \mathrm{ppm}$.

${ }^{13} \mathbf{C}$ NMR $\left(75 \mathrm{MHz}, \mathrm{CDCl}_{3}\right) \delta=210.7,158.0,133.3,129.3,114.0,55.4,44.7,42.9,29.1$, 26.0, 22.4, $14.0 \mathrm{ppm}$

MS (EI, $70 \mathrm{eV}) \mathrm{m} / z: 220.1\left(\mathrm{M}^{+}\right), 163.1,135.1,121.0,108.0,91.1,85.0,77.0,57.1$.

HRMS (ESI+): $m / z,\left[\mathrm{C}_{14} \mathrm{H}_{20} \mathrm{O}_{2}+\mathrm{H}\right]^{+}$calcd. 221.15361, found 221.15303.

3-(4-methoxyphenyl)-1-(pyridin-2-yl)propan-1-one (7b)
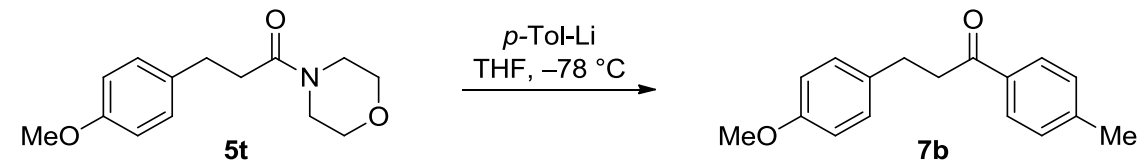

To a solution of para-tolyl lithium (241 mg, $2.46 \mathrm{mmol}, 3$ eq.) in abs. THF ( $8 \mathrm{~mL}$ ) was added a solution of 3-(4-methoxyphenyl)-1-morpholinopropan-1-one (204 mg, $0.819 \mathrm{mmol}, 1$ eq.) in abs. THF $(3 \mathrm{~mL})$ dropwise by syringe at $-78{ }^{\circ} \mathrm{C}$. The reaction mixture was stirred for 1 hour and was then quenched by the addition of aqueous acetic acid $(30 \%, 5 \mathrm{~mL})$. The reaction mixture was warmed to $\mathrm{rt}$ and extracted with $\operatorname{MTBE}(3 \mathrm{x} 30 \mathrm{~mL})$. The combined organic phase was dried over $\mathrm{Na}_{2} \mathrm{SO}_{4}$ and concentrated. Column chromatography (pentane/diethyl ether, 
9:1) gave the title compound as a colorless oil which solidified upon standing (187 mg, 0.736 mmol, $90 \%)$.

${ }^{1} \mathbf{H}$ NMR $\left(500 \mathrm{MHz}, \mathrm{CDCl}_{3}\right) \delta=7.79(\mathrm{~d}, J=8.24 \mathrm{~Hz}, 2 \mathrm{H}), 7.14-7.23(\mathrm{~m}, 2 \mathrm{H}), 7.05-7.13$ (m, 2 H), 6.72 - 6.81 (m, 2 H), 3.71 (s, $3 \mathrm{H}), 3.11$ - 3.23 (m, $2 \mathrm{H}), 2.89$ - 2.97 (m, $2 \mathrm{H}), 2.33$ (s, $3 \mathrm{H}) \mathrm{ppm}$.

${ }^{13}$ C NMR $\left(126 \mathrm{MHz}, \mathrm{CDCl}_{3}\right) \delta=199.1,158.0,143.9,134.5,133.5,129.4,128.3,114.0,55.3$, 40.7, 29.4, $21.7 \mathrm{ppm}$.

MS (EI, $70 \mathrm{eV}) m / z: 254.1\left(\mathrm{M}^{+}\right), 239.1,135.0,121.1,108.1,91.1,77.0,65.1,51.0$.

The spectroscopic data correspond to those reported in the literature. ${ }^{12}$

3-(4-methoxyphenyl)-1-(pyridin-2-yl)propan-1-one (7c)<smiles>COc1ccc(CCC(=O)N2CCOCC2)cc1</smiles>

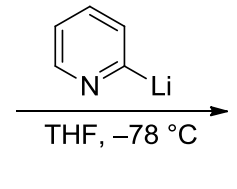<smiles>COc1ccc(CCC(=O)c2ccccn2)cc1</smiles>

7c

To a solution of 2-bromopyridine (438 mg, $264 \mu \mathrm{L}, 2.77 \mathrm{mmol}, 3.0 \mathrm{eq})$ in abs. THF (4 mL) was added $n$-butyl lithium ( $1.6 \mathrm{M}$ in hexane, $1.44 \mathrm{~mL}, 2.30 \mathrm{mmol}, 2.5 \mathrm{eq})$ dropwise at $-78{ }^{\circ} \mathrm{C}$ and the resulting dark-orange solution was stirred for 1 hour at this temperature. Then a solution of the amide $5 \mathrm{t}$ ( $230 \mathrm{mg}, 0.924 \mathrm{mmol}, 1.0 \mathrm{eq})$ in THF ( $3 \mathrm{~mL})$ was added dropwise. After one hour the reaction was quenched by the addition of hydrochloric acid $(1 \mathrm{M}, 5 \mathrm{~mL})$, diluted with MTBE and warmed to rt. The aqueous phase was extracted with MTBE (3x 30 $\mathrm{mL}$ ) and the combined organic phase was dried over $\mathrm{Na}_{2} \mathrm{SO}_{4}$ and concentrated. Column chromatography (silica gel, pentane/diethyl ether, 5:1) gave the ketone as a colorless oil (180 mg, $0.747 \mathrm{mmol}, 81 \%)$.

${ }^{1} \mathbf{H}$ NMR $\left(500 \mathrm{MHz}, \mathrm{CDCl}_{3}\right) \delta=8.58-8.73(\mathrm{~m}, 1 \mathrm{H}), 8.03(\mathrm{dt}, J=7.78,1.14 \mathrm{~Hz}, 1 \mathrm{H}), 7.82$ $(\mathrm{td}, J=7.71,1.68 \mathrm{~Hz}, 1 \mathrm{H}), 7.45$ (ddd, $J=7.63,4.88,1.22 \mathrm{~Hz}, 1 \mathrm{H}), 7.12$ - 7.24 (m, $2 \mathrm{H}), 6.76$ 6.89 (m, $2 \mathrm{H}), 3.77$ (s, $3 \mathrm{H}), 3.54$ (t, $J=7.63 \mathrm{~Hz}, 2 \mathrm{H}), 3.01$ (t, J=7.63 Hz, $2 \mathrm{H}) \mathrm{ppm}$.

${ }^{13} \mathrm{C}$ NMR (126 MHz, $\left.\mathrm{CDCl}_{3}\right) \delta=201.2,158.0,153.4,149.1,137.0,133.6,129.5,127.2$, 121.9, 113.9, 55.3, 39.8, $29.1 \mathrm{ppm}$.

MS (EI, $70 \mathrm{eV}) \mathrm{m} / z: 241.1\left(\mathrm{M}^{+}\right), 212.1,121.1,107.0,91.1,79.0,65.1,51.0$.

HRMS (ESI+): $m / z\left[\mathrm{C}_{10} \mathrm{H}_{14} \mathrm{~N}_{2} \mathrm{O}+\mathrm{H}\right]^{+}$calcd. 242.11756, found 242.11702

1-cyclopropylheptan-3-one $(7 \mathrm{~d})$<smiles>O=C(CCC1CC1)N1CCOCC1</smiles>

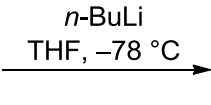

$5 v$

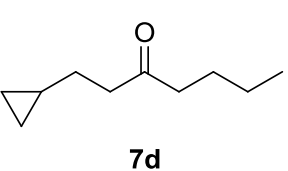

To a solution of amide $\mathbf{5 v}(267 \mathrm{mg}, 1.46 \mathrm{mmol}, 1 \mathrm{eq})$ in abs. THF (5 mL) was added $n$-butyl lithium (1.6 $\mathrm{M}$ in hexane, $2.73 \mathrm{~mL}, 4.38 \mathrm{mmol}, 3 \mathrm{eq})$ dropwise at $-78{ }^{\circ} \mathrm{C}$. The reaction mixture was stirred at this temperature for 1 hour and was then quenched with hydrochloric 
acid $(1 \mathrm{M}, 5 \mathrm{~mL})$ and diluted with diethyl ether $(10 \mathrm{~mL})$. After warming to $\mathrm{rt}$, the reaction mixture was extracted with diethyl ether $(3 \times 10 \mathrm{~mL})$, the combined phase was dried over $\mathrm{Na}_{2} \mathrm{SO}_{4}$ and concentrated. Column chromatography (pentane/diethyl ether, 15:1, $1^{\text {st }}$ eluting fraction) gave ketone 7d as a colorless oil (203 mg, $1.32 \mathrm{mmol}, 90 \%)$.

${ }^{1} \mathbf{H}$ NMR $\left(500 \mathrm{MHz}, \mathrm{CDCl}_{3}\right) \delta=2.50(\mathrm{t}, J=7.32 \mathrm{~Hz}, 2 \mathrm{H}), 2.41(\mathrm{t}, J=7.48 \mathrm{~Hz}, 2 \mathrm{H}), 1.55$ (dt, $J=15.18,7.51 \mathrm{~Hz}, 2 \mathrm{H}), 1.46(\mathrm{q}, J=7.12 \mathrm{~Hz}, 2 \mathrm{H}), 1.31$ (dq, $J=15.03,7.40 \mathrm{~Hz}, 2 \mathrm{H}), 0.90$ (t, $J=7.32 \mathrm{~Hz}, 3 \mathrm{H}), 0.61-0.71(\mathrm{~m}, 1 \mathrm{H}), 0.35-0.46(\mathrm{~m}, 2 \mathrm{H}),-0.01-0.05$ (m, $2 \mathrm{H}) \mathrm{ppm}$.

${ }^{13} \mathbf{C ~ N M R}\left(126 \mathrm{MHz}, \mathrm{CDCl}_{3}\right) \delta=211.8,42.9,29.2,26.1,22.5,14.0,10.7,4.6 \mathrm{ppm}$.

MS (EI, $70 \mathrm{eV}) \mathrm{m} / z: 154.1\left(\mathrm{M}^{+}\right), 139.1,125.1,112.1,97.1,83.1,69.1,57.1$.

Elemental analysis $(\%)$ for $\mathrm{C}_{10} \mathrm{H}_{18} \mathrm{O}$ : calcd. $\mathrm{C} 77.87, \mathrm{H}$ 11.76; found: $\mathrm{C} 77.76, \mathrm{H} 12.03$.

Due to low molecular weight and polarity, no ESI+ HRMS could be obtained.

\section{3-(4-methoxyphenyl)propanal (7e)}

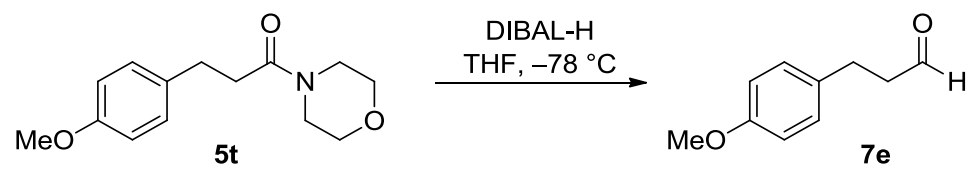

To a solution of amide $\mathbf{5 t}(130 \mathrm{mg}, 0.522 \mathrm{mmol}, 1 \mathrm{eq})$ in abs. THF ( $3 \mathrm{~mL})$ was added DIBAL$\mathrm{H}(1 \mathrm{M}$ in hexane, $0.6 \mathrm{~mL}, 0.600 \mathrm{mmol}, 1.15 \mathrm{eq})$ at $-78{ }^{\circ} \mathrm{C}$. The reaction was stirred for 50 min and was then quenched by the addition of an aqueous solution of $\mathrm{Na} / \mathrm{K}$ tartrate and diluted with diethyl ether. The reaction was warmed to $\mathrm{rt}$, the phases were separated and the aqueous phase was extracted with diethyl ether $(4 \mathrm{x} 20 \mathrm{~mL})$. The combined organic phase was dried over $\mathrm{Na}_{2} \mathrm{SO}_{4}$ and concentrated. Column chromatography (pentane/diethyl ether, 1:1) gave the aldehyde as a colorless oil (81 $\mathrm{mg}, 0.494 \mathrm{mmol}, 95 \%)$.

${ }^{1} \mathbf{H}$ NMR $\left(300 \mathrm{MHz}, \mathrm{CDCl}_{3}\right) \delta=9.81(\mathrm{t}, J=1.46 \mathrm{~Hz}, 1 \mathrm{H}), 7.03-7.18(\mathrm{~m}, 2 \mathrm{H}), 6.75-6.94$ (m, $2 \mathrm{H}), 3.78$ (s, $3 \mathrm{H}), 2.86$ - 2.95 (m, $2 \mathrm{H}), 2.69$ - 2.78 (m, $2 \mathrm{H}) \mathrm{ppm}$.

${ }^{13}$ C NMR $\left(75 \mathrm{MHz}, \mathrm{CDCl}_{3}\right) \delta=201.9,158.2,129.3,114.1,55.4,45.7,27.4 \mathrm{ppm}$. MS (EI, $70 \mathrm{eV}) \mathrm{m} / z: 164.1\left(\mathrm{M}^{+}\right), 121.1,108.1,91.1,77.1,65.1$.

The spectroscopic data correspond to those reported in the literature. ${ }^{13}$

5-phenylpentanal (7f)
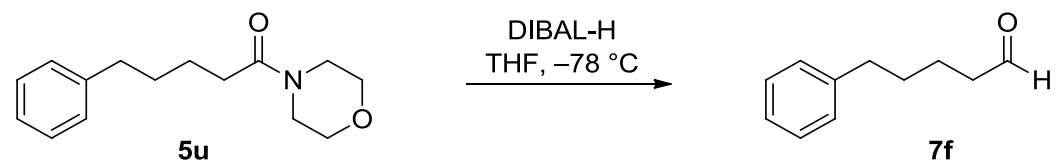

To a solution of amide $5 \mathbf{u}(245 \mathrm{mg}, 0.991 \mathrm{mmol}, 1 \mathrm{eq})$ in abs. THF (5 mL) was added DIBAL-H ( $1 \mathrm{M}$ in hexane, $1.09 \mathrm{~mL}, 1.09 \mathrm{mmol}, 1.1 \mathrm{eq})$ dropwise at $-78{ }^{\circ} \mathrm{C}$. After 1 hour the reaction was monitored by TLC and another aliquot of DIBAL-H (300 $\mu \mathrm{L}, 300 \mu \mathrm{mol})$ was added. After further $20 \mathrm{~min}$ the reaction mixture was quenched with an aqueous solution of citric acid (33 wt-\%, $6 \mathrm{~mL})$, diluted with diethyl ether $(10 \mathrm{~mL})$, warmed to $\mathrm{rt}$ and stirred for 
$30 \mathrm{~min}$. The phases were separated and the aqueous phase was extracted with diethyl ether ( 3 x $10 \mathrm{~mL}$ ). The combined organic phase was dried over $\mathrm{Na}_{2} \mathrm{SO}_{4}$ and concentrated. Column chromatography (pentane/diethyl ether, 15:1) gave the aldehyde $7 \mathbf{f}$ as a colorless oil (117 mg, $0.722 \mathrm{mmol}, 73 \%)$.

The ${ }^{1} \mathrm{H}$ NMR spectrum was referenced using dichloromethane $(\delta=5.30 \mathrm{ppm})$ because compound- and $\mathrm{CDCl}_{3}$ signals overlapped.

${ }^{1} \mathbf{H}$ NMR $\left(500 \mathrm{MHz}, \mathrm{CDCl}_{3}\right) \delta=9.76(\mathrm{t}, J=1.83 \mathrm{~Hz}, 1 \mathrm{H}), 7.24-7.33(\mathrm{~m}, 2 \mathrm{H}), 7.13-7.24$ (m, $3 \mathrm{H}), 2.57$ - 2.70 (m, $2 \mathrm{H}), 2.46$ (dtd, J=7.06, 3.57, 3.57, $1.68 \mathrm{~Hz}, 2 \mathrm{H}), 1.62-1.75$ (m, 4 H) ppm.

${ }^{13}$ C NMR $\left(126 \mathrm{MHz}, \mathrm{CDCl}_{3}\right) \delta=202.7,142.1,128.5,126.0,43.9,35.8,31.0,21.8 \mathrm{ppm}$.

MS (EI, $70 \mathrm{eV}) \mathrm{m} / \mathrm{z}: 162.1\left(\mathrm{M}^{+}\right), 144.1,129.1,117.1,105.1,91.1,84.1,77.1,71.1,65.1,57.1$, 51.1.

The spectroscopic data correspond to those reported in the literature. ${ }^{14}$ 


\section{NMR-Spectra}

ND-522-1-SC.010.esp

Frequency (MHz): 500.13

Solvent: CHLOROFORM-d<smiles>CN(C)C(=O)CCc1ccccc1</smiles>

$5 a$
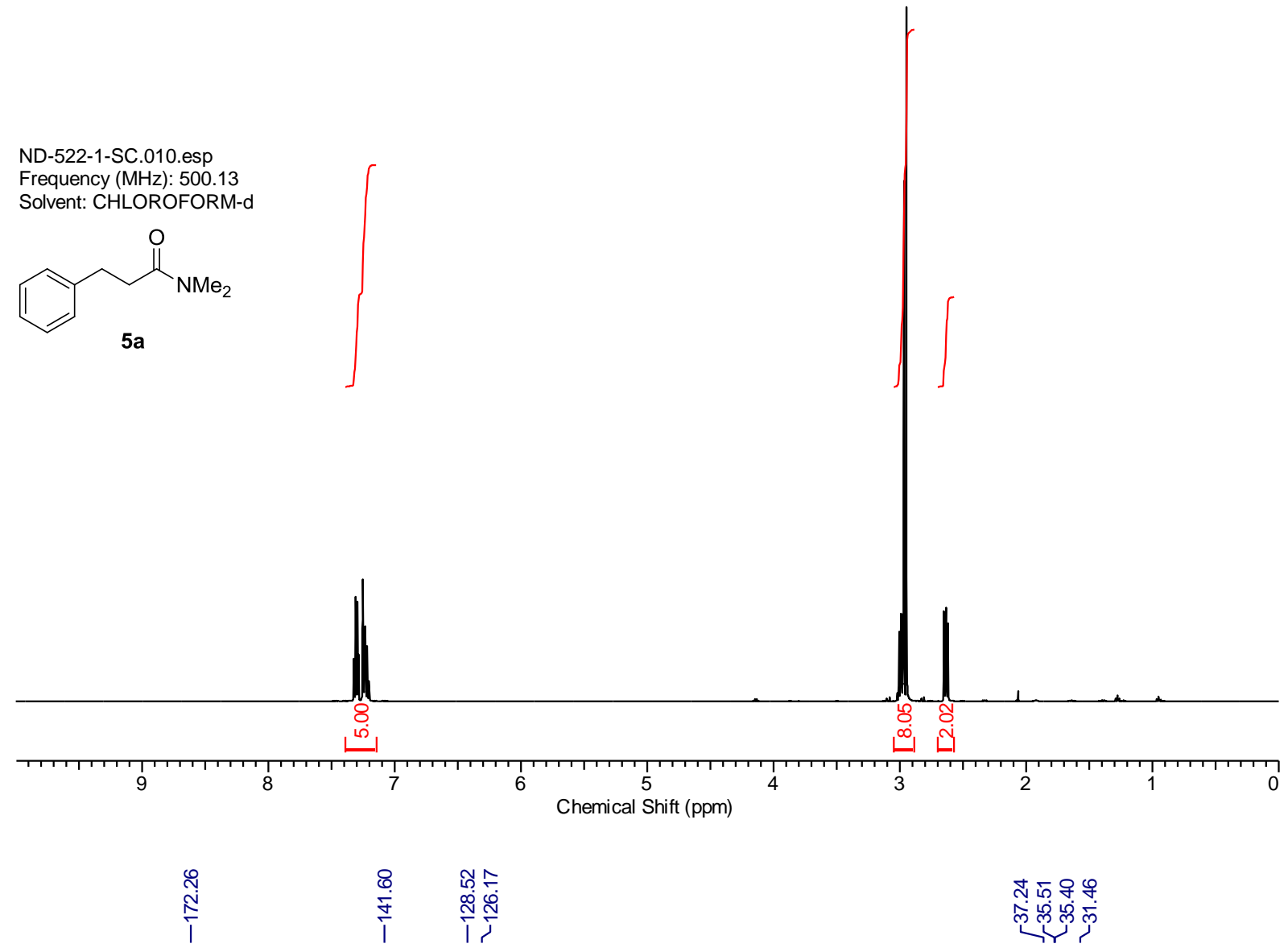

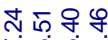

ஸิ ผึं ผึं

ND-522-1-SC.011.esp

Frequency (MHz): 125.76

Solvent: CHLOROFORM-d

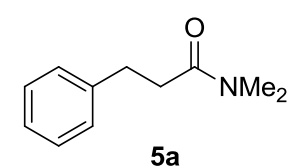

$5 a$

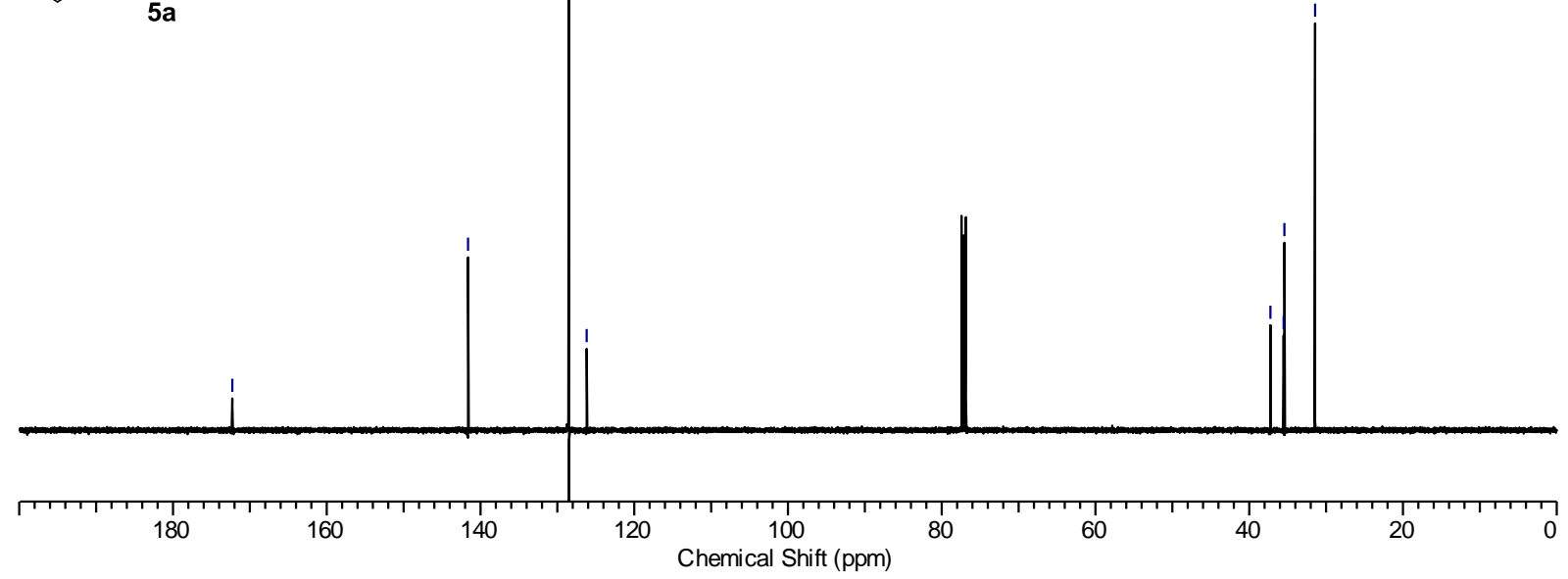


ND-532-7.010.esp

Frequency (MHz): 500.13

Solvent: CHLOROFORM-d
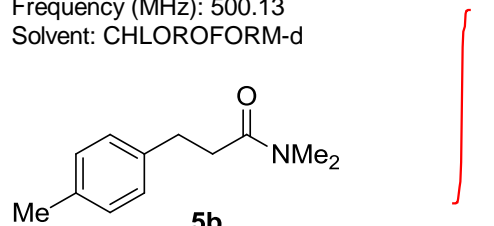

$5 b$
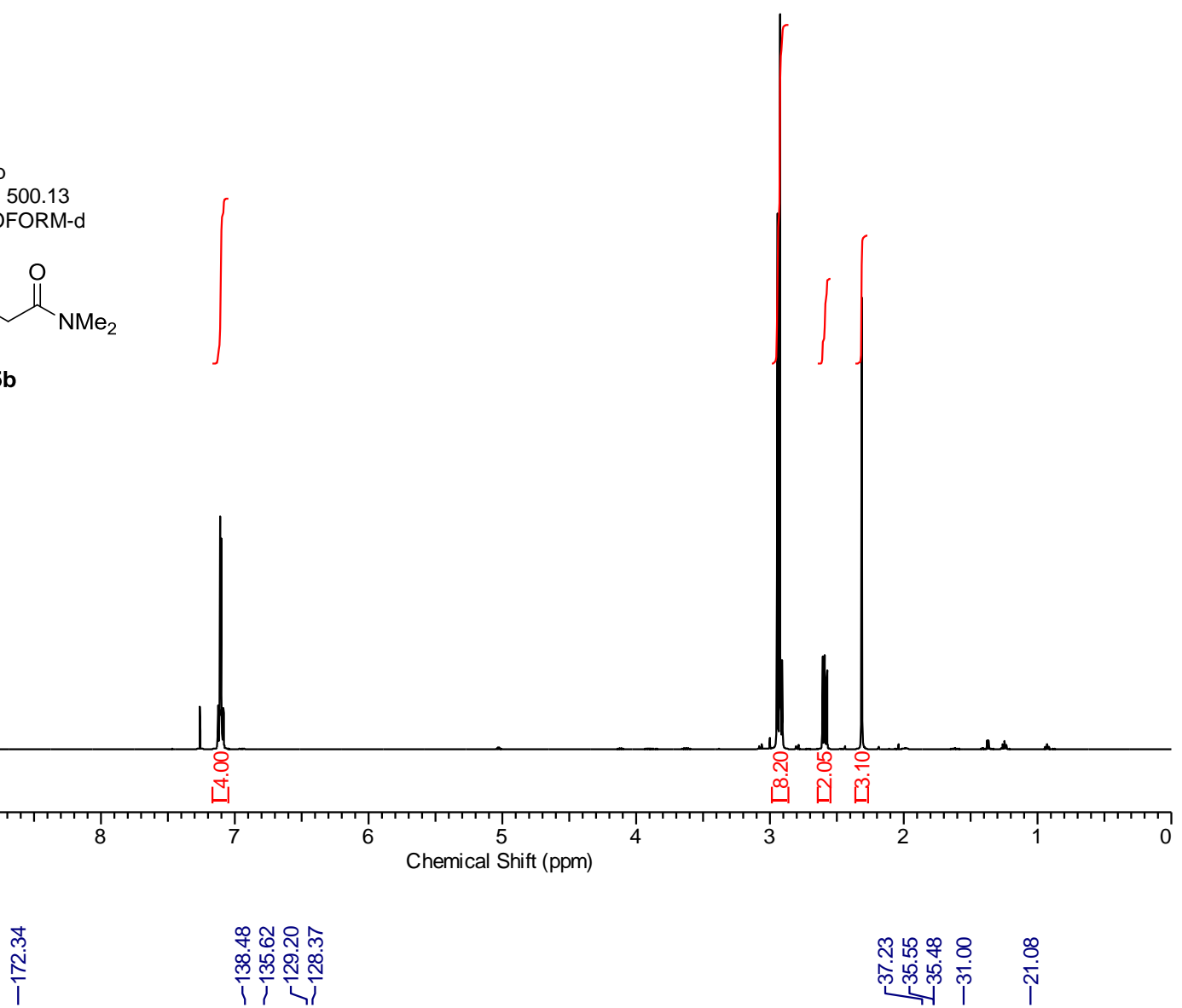

స్ํํㅇ \&

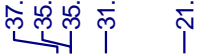

ND-532-7.011.esp

Frequency (MHz): 125.76

Solvent: CHLOROFORM-d<smiles>Cc1ccc(CCC(=O)N(C)C)cc1</smiles>

$5 b$

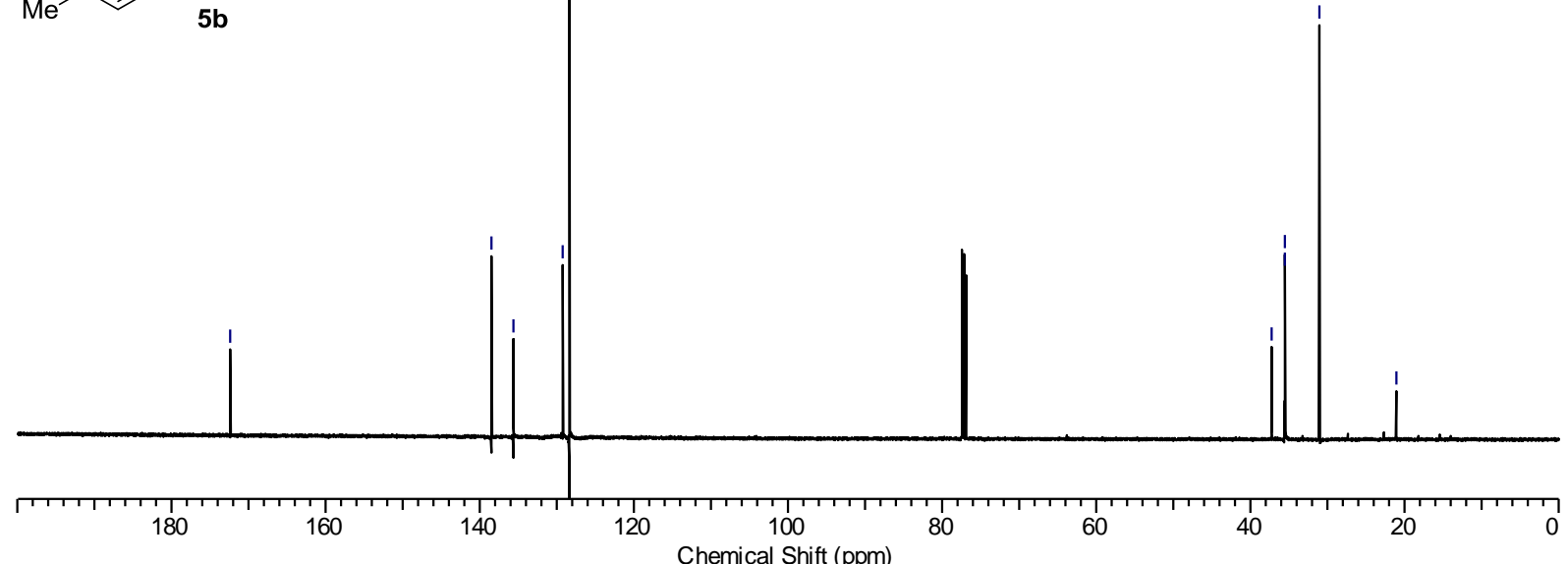


ND-532-9.010.esp

Frequency $(\mathrm{MHz}): 500.13$

Solvent: CHLOROFORM-d<smiles>Cc1cccc(CCC(=O)N(C)C)c1</smiles>

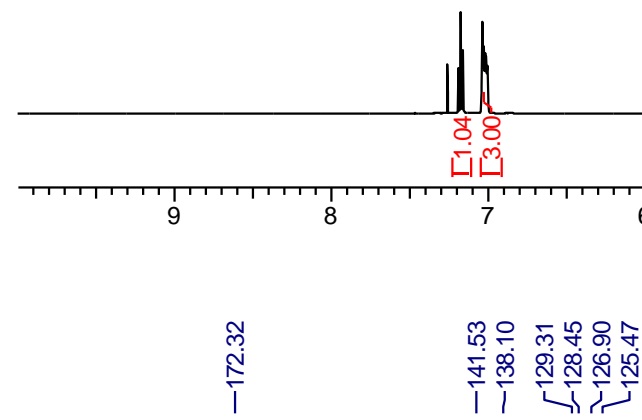

ND-532-9.011.esp

Frequency (MHz): 125.76

Solvent: CHLOROFORM-d<smiles>Cc1cccc(CCC(=O)N(C)C)c1</smiles>

Chemical Shift (ppm)

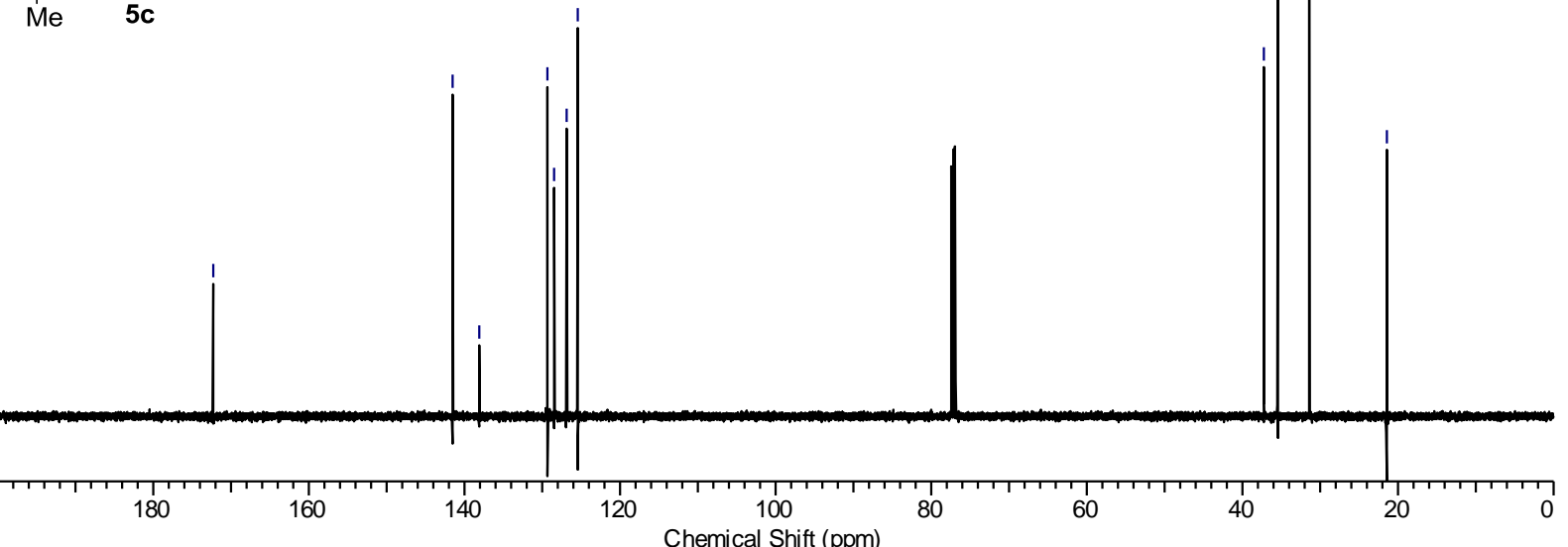


ND-536-2.010.esp

Frequency (MHz): 500.13

Solvent: CHLOROFORM-d
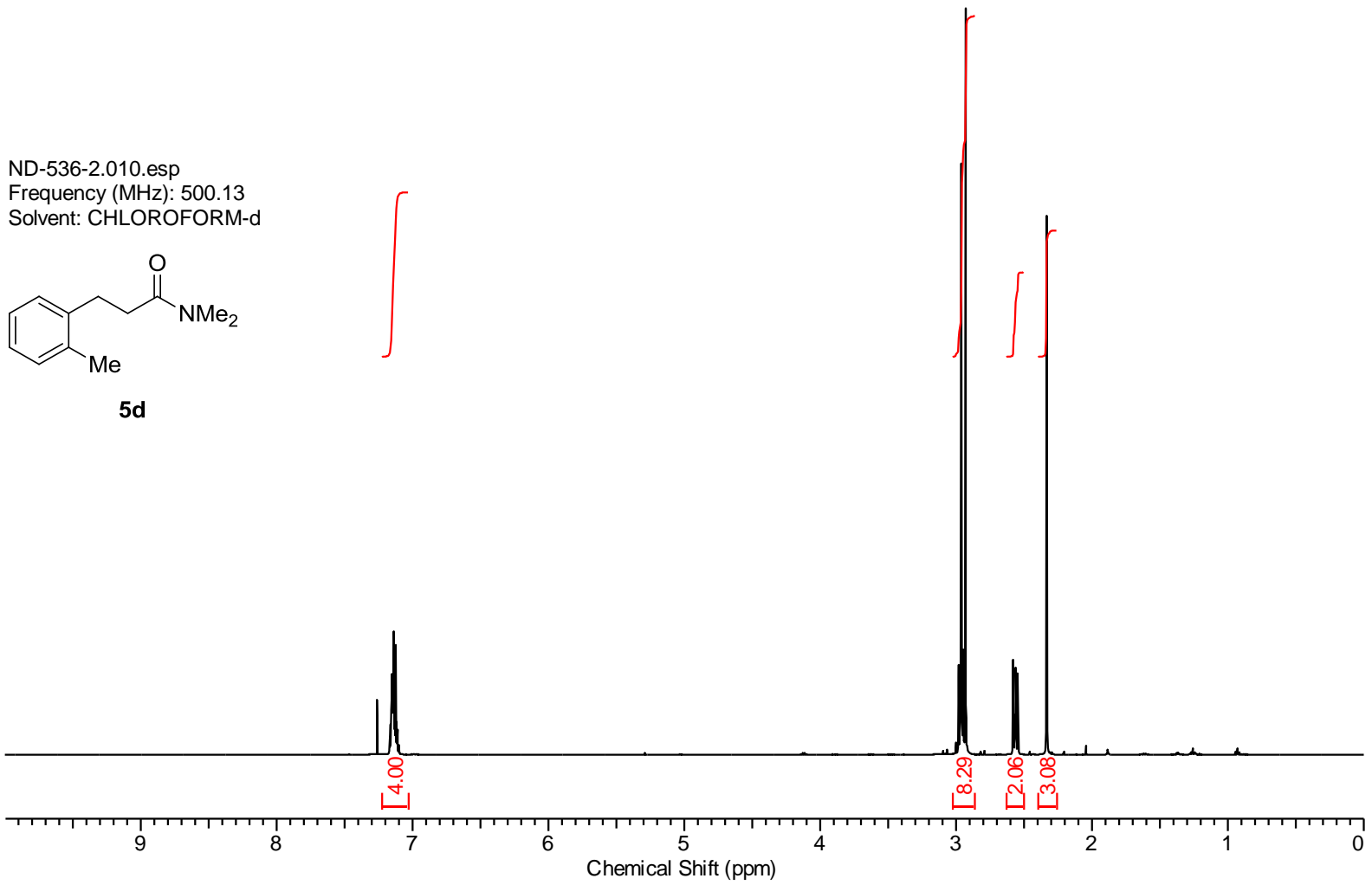

5d

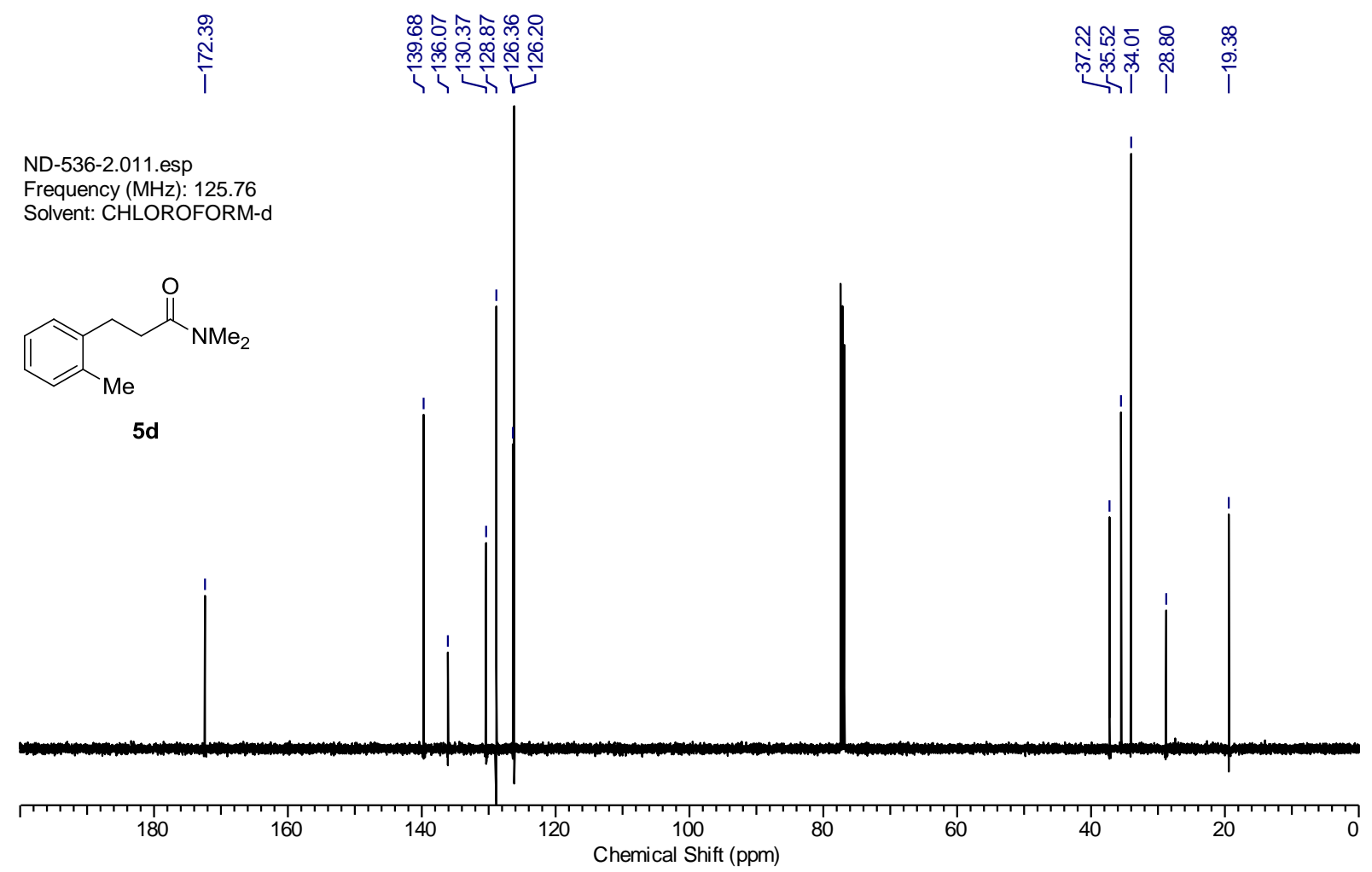


ND-536-1.010.esp

Frequency (MHz): 500.13

Solvent: CHLOROFORM-d
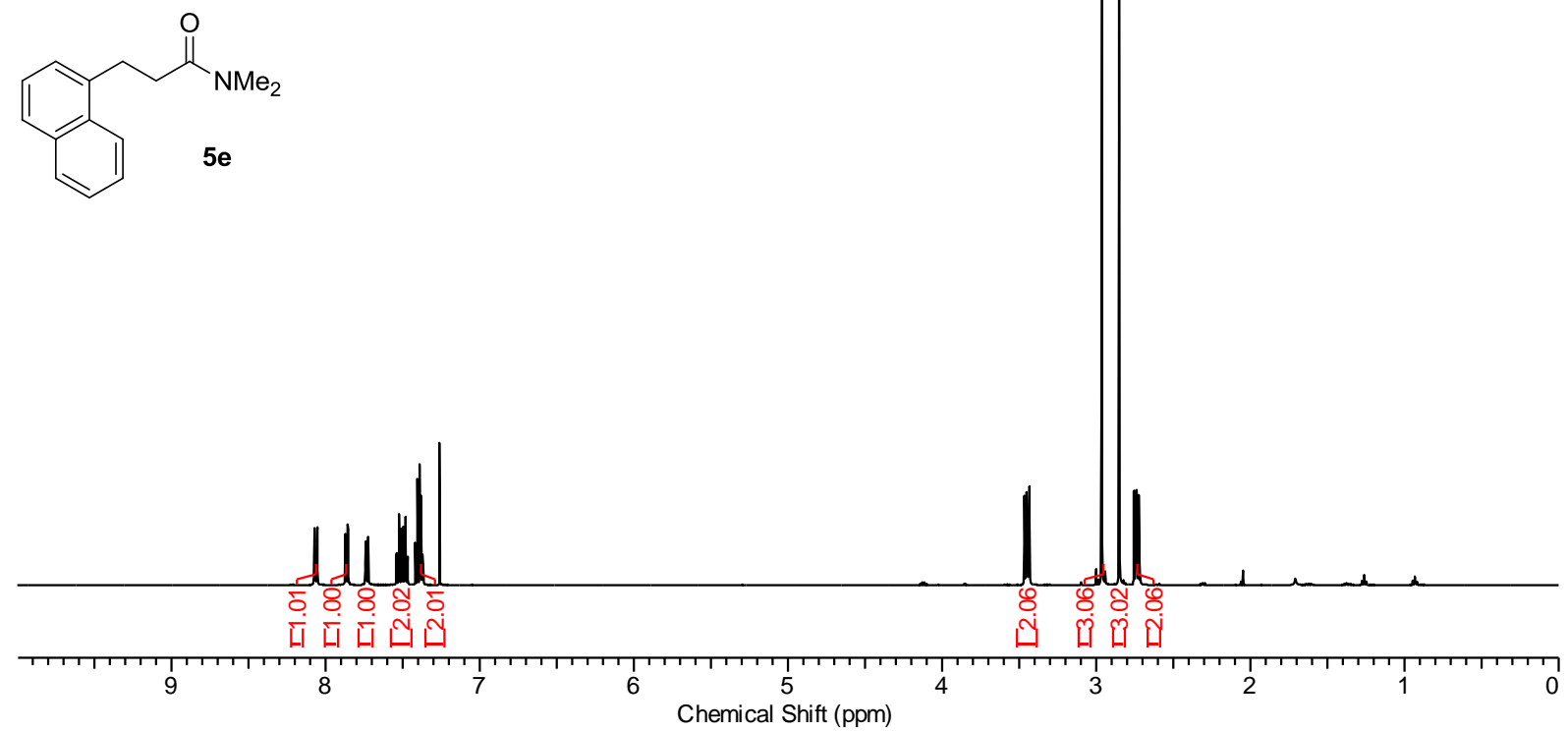

กุ

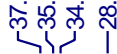

ND-536-1.011.esp

Frequency (MHz): 125.76

Solvent: CHLOROFORM-d<smiles>CN(C)C(=O)CCc1cccc2ccccc12</smiles>

$5 e$

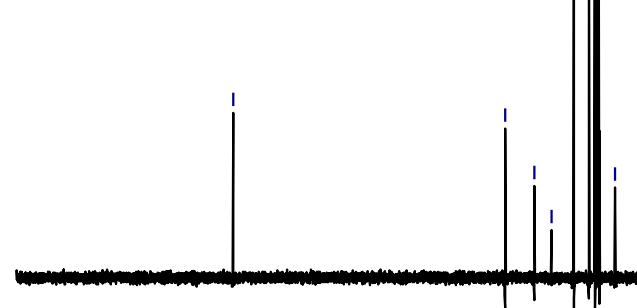


ND-532-8.010.esp

Frequency (MHz): 500.13

Solvent: CHLOROFORM-d
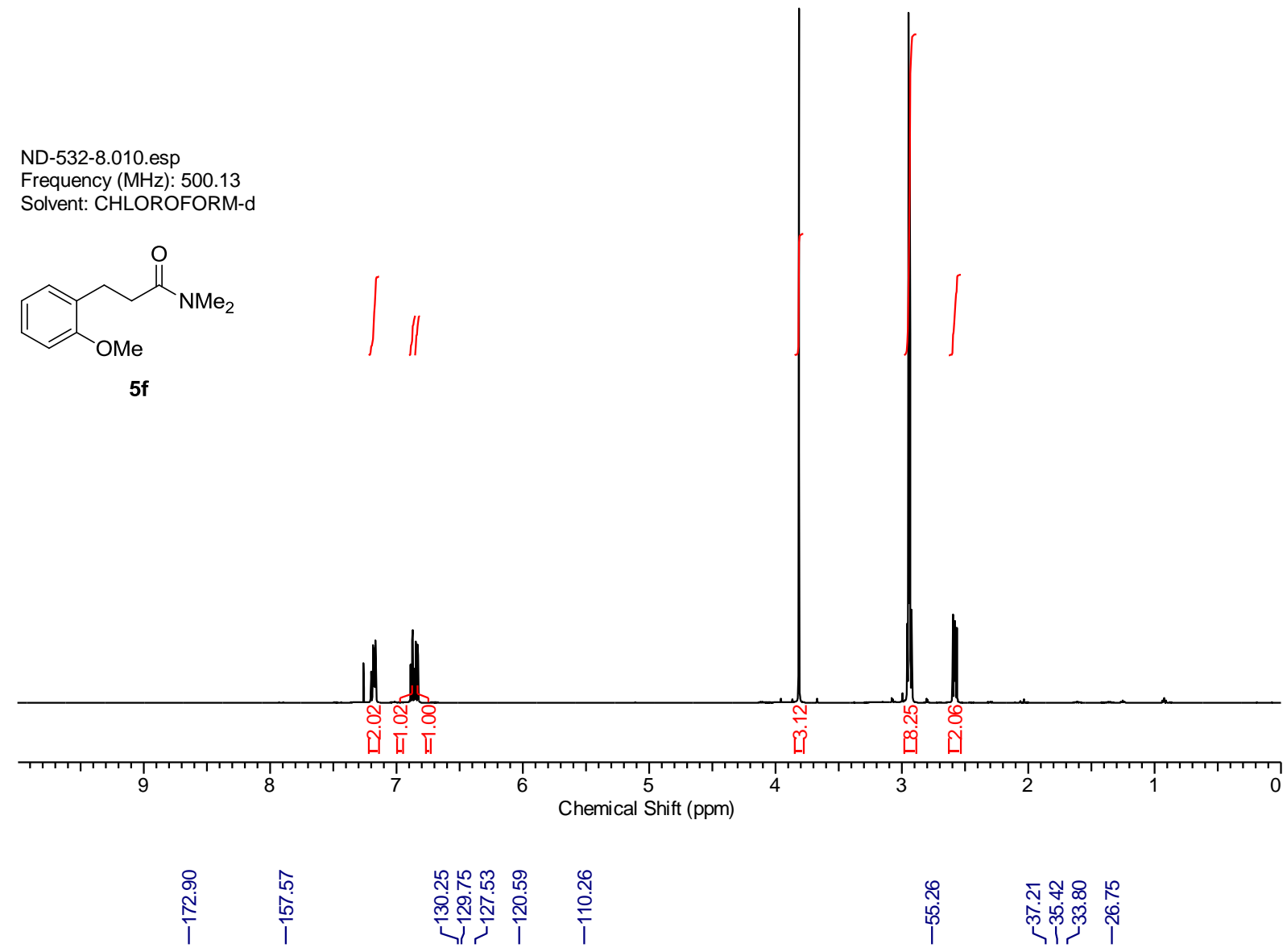

ND-532-8.011.esp

Frequency (MHz): 125.76

Solvent: CHLOROFORM-d<smiles>COc1ccccc1CCC(=O)N(C)C</smiles>

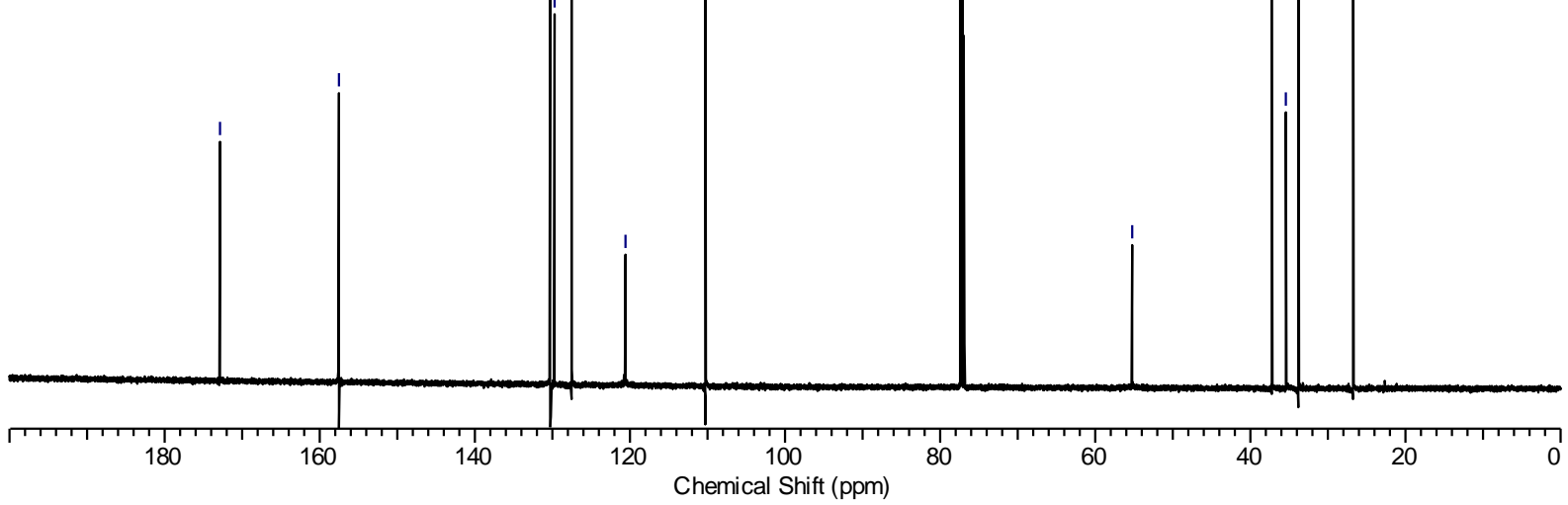


ND-522-2-SC.010.esp

Frequency (MHz): 500.13

Solvent: CHLOROFORM-d
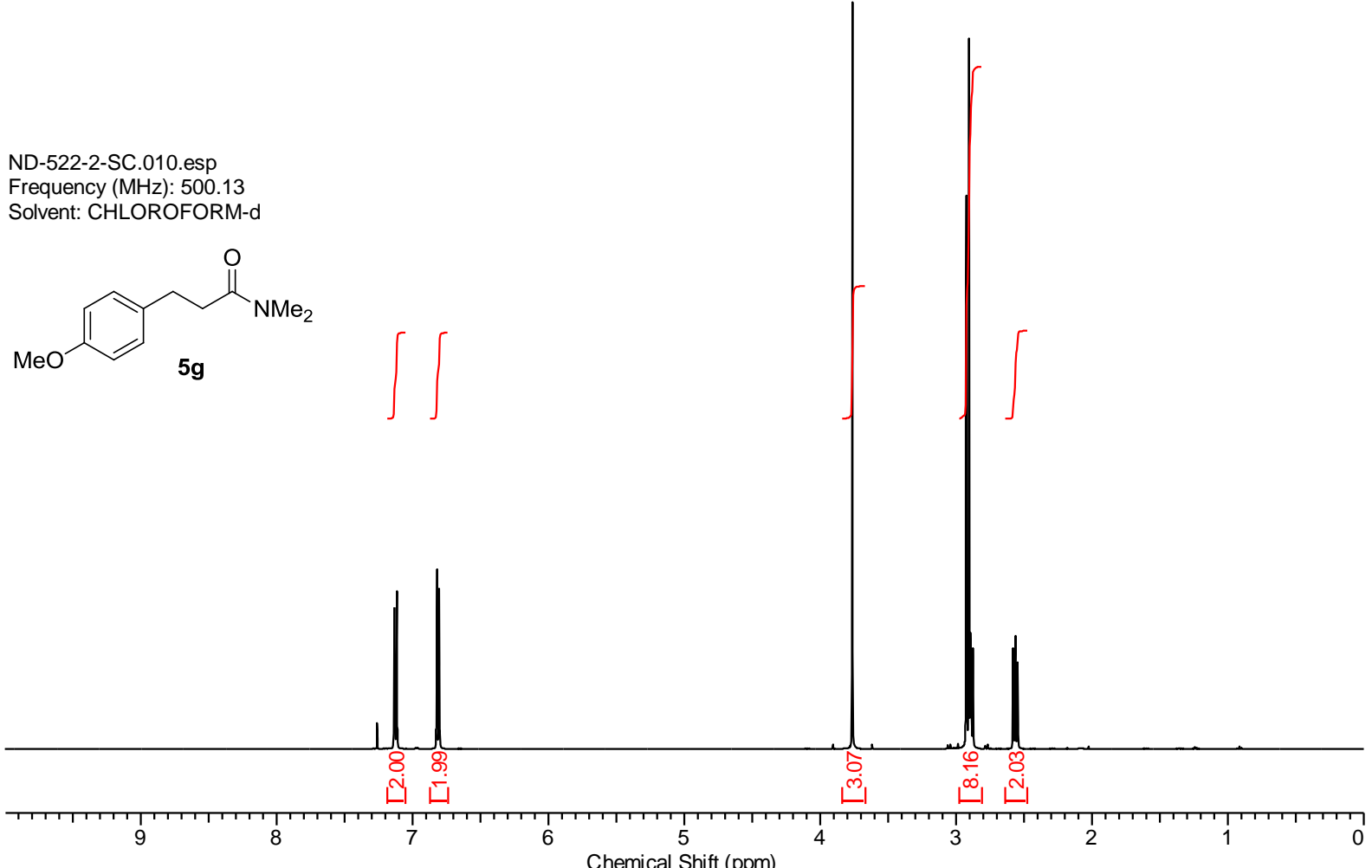

\begin{tabular}{|c|c|c|}
\hline $\begin{array}{l}\bar{m} \\
\stackrel{N}{N} \\
\end{array}$ & $\begin{array}{l}\bar{\sigma} \\
\infty \\
\stackrel{0}{0} \\
\text { j. }\end{array}$ & 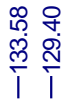 \\
\hline
\end{tabular}

న

ND-522-2-SC.020.esp

Frequency (MHz): 125.76

Solvent: CHLOROFORM-d<smiles>COc1ccc(CCC(C)=O)cc1</smiles>

$5 g$

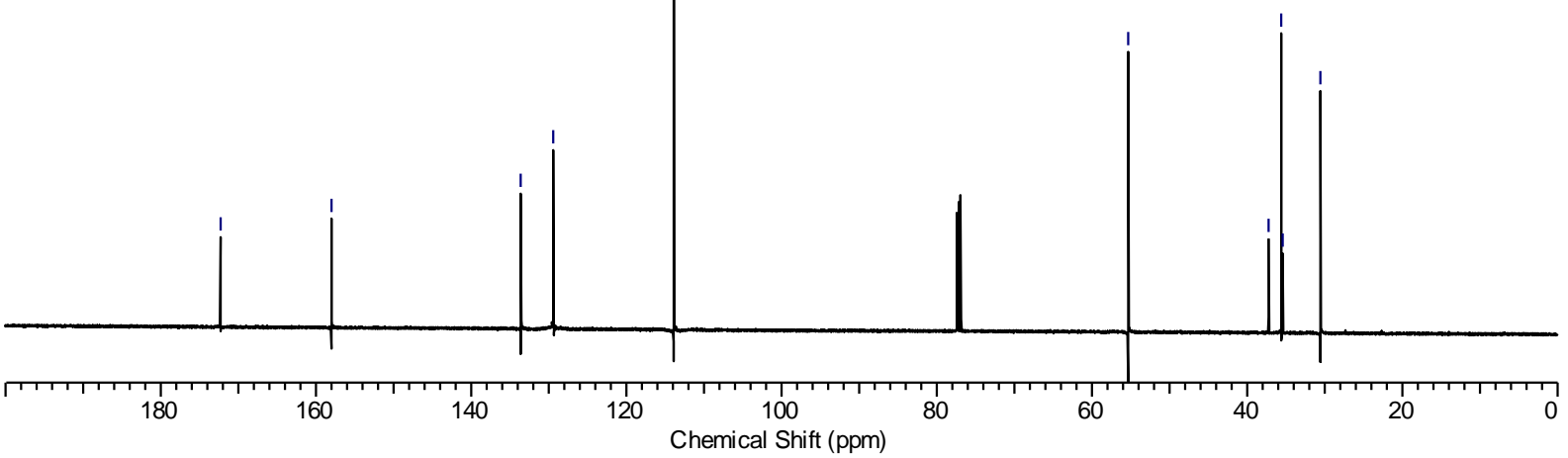


ND-582-1-SC.010.esp

Frequency (MHz): 500.13

Solvent: CHLOROFORM-d
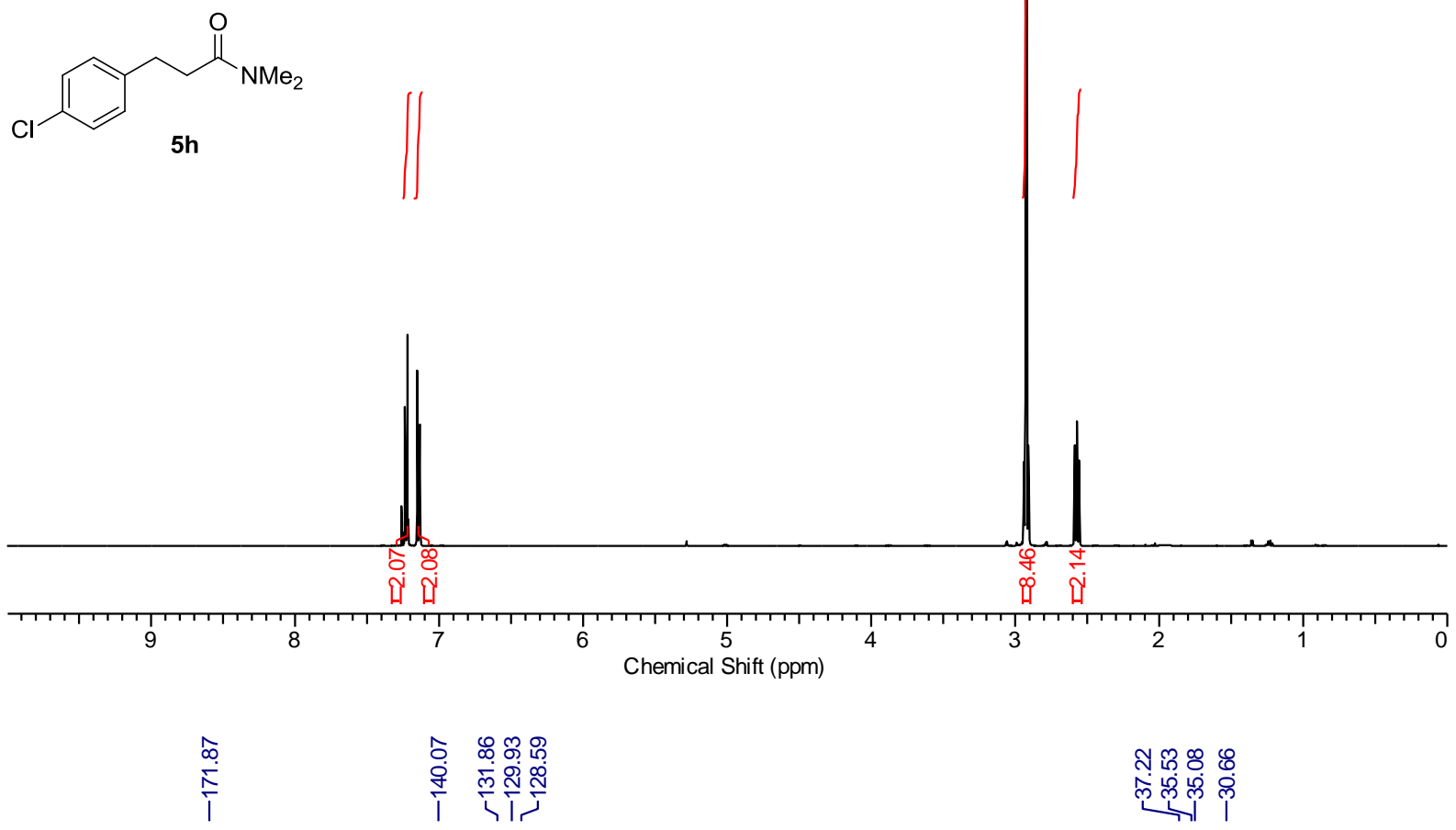

สำ

लू लूं क्

ND-582-1-SC.011.esp

Frequency (MHz): 125.76

Solvent: CHLOROFORM-d<smiles>CN(C)C(=O)CCc1ccc(Cl)cc1</smiles>

$5 h$

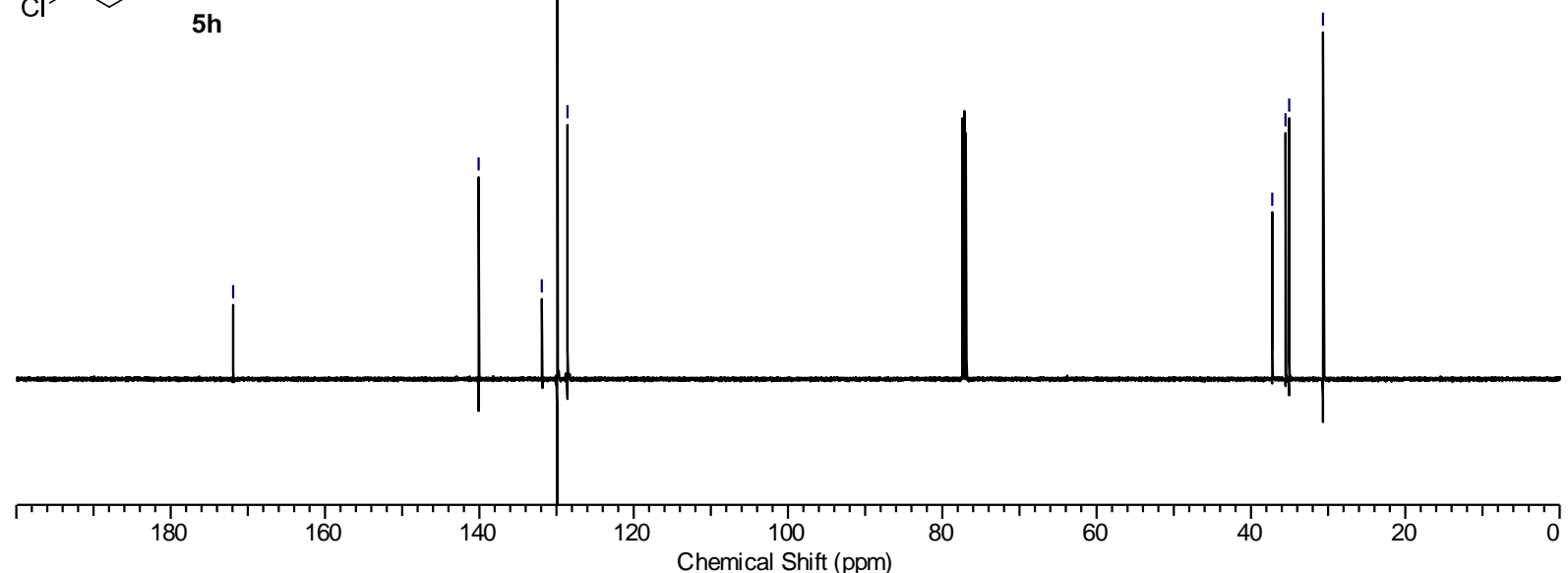




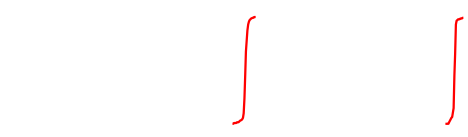<smiles>CN(C)C(=O)CCc1ccncc1</smiles>

$5 \mathbf{i}$
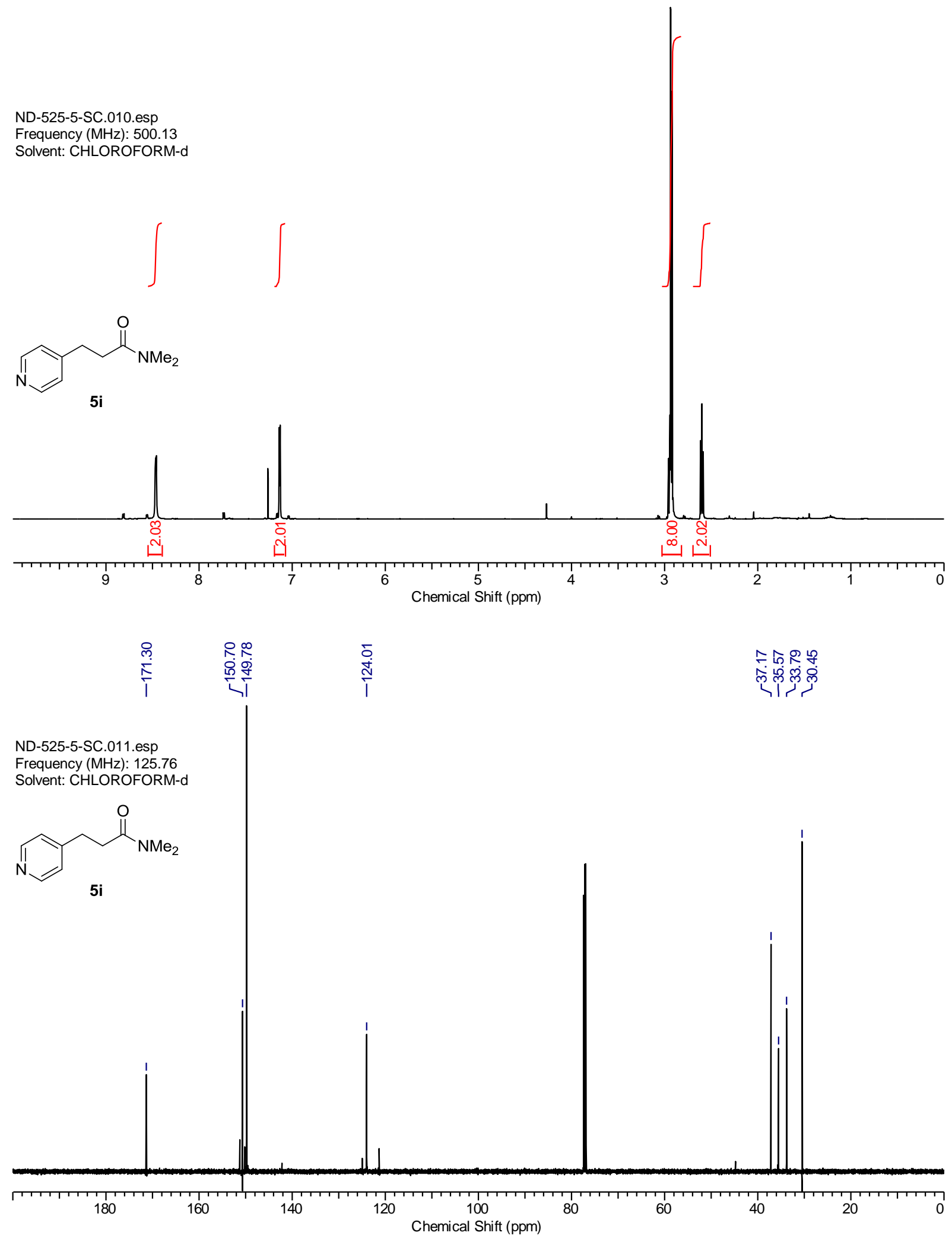
ND-524-2-SC.010.esp

Frequency (MHz): 500.13

Solvent: CHLOROFORM-d<smiles>CNC(=O)CCc1cccnc1</smiles><smiles>[CH]/C=C\CC</smiles>

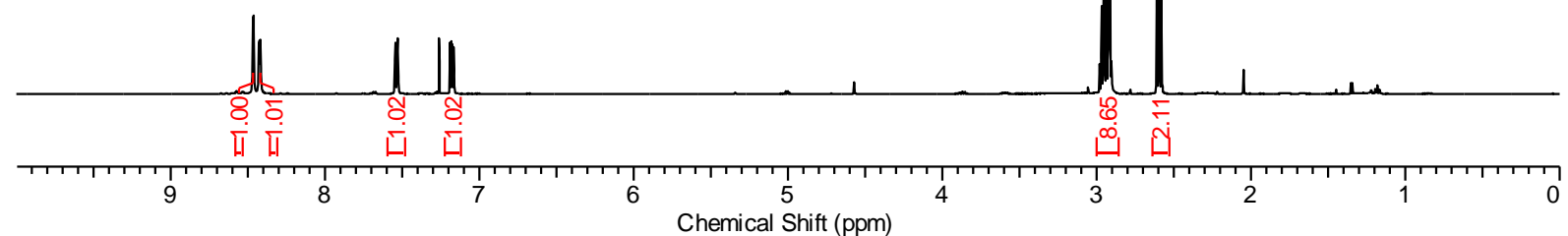

ND-524-2-SC.011.esp

Frequency (MHz): 125.76

Solvent: CHLOROFORM-d<smiles>CNC(=O)CCc1cccnc1</smiles>

5j

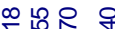

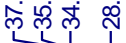

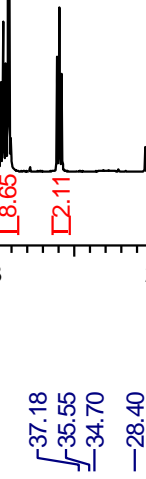

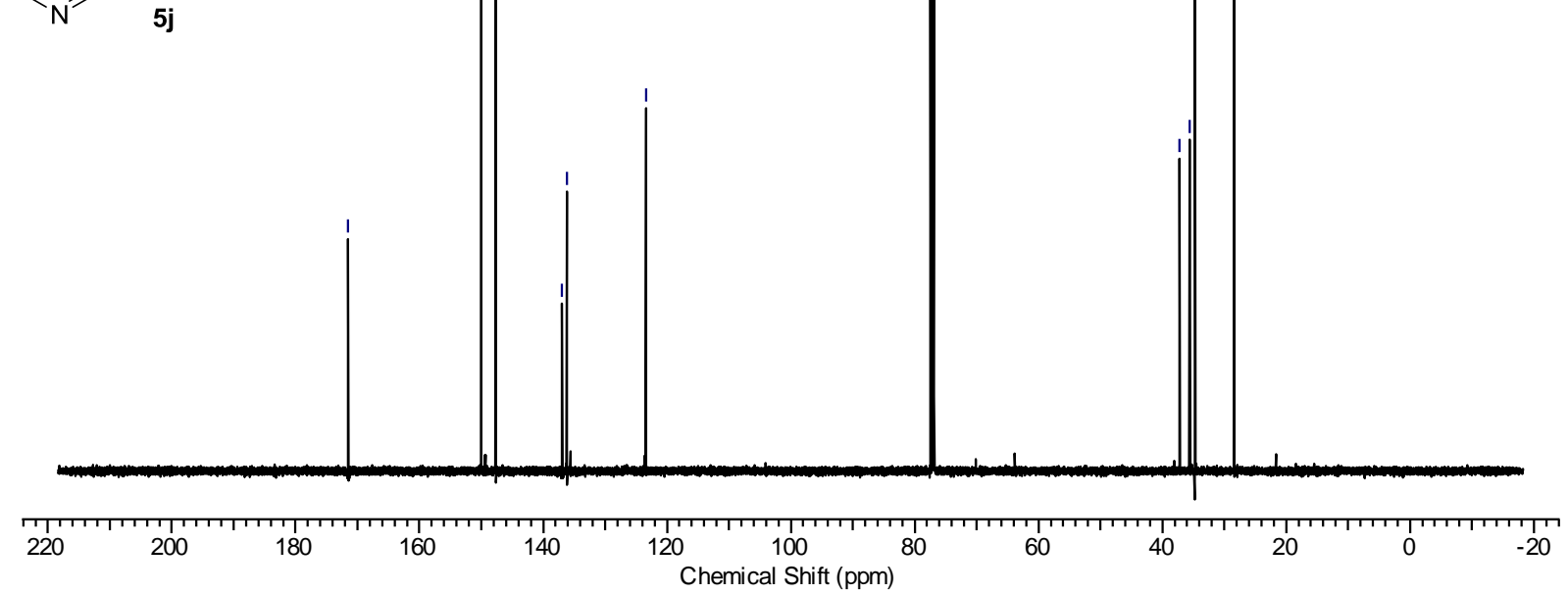


Solvent: CHLOROFORM-d

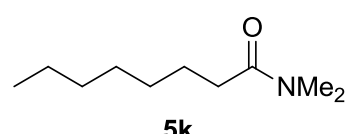

$5 k$

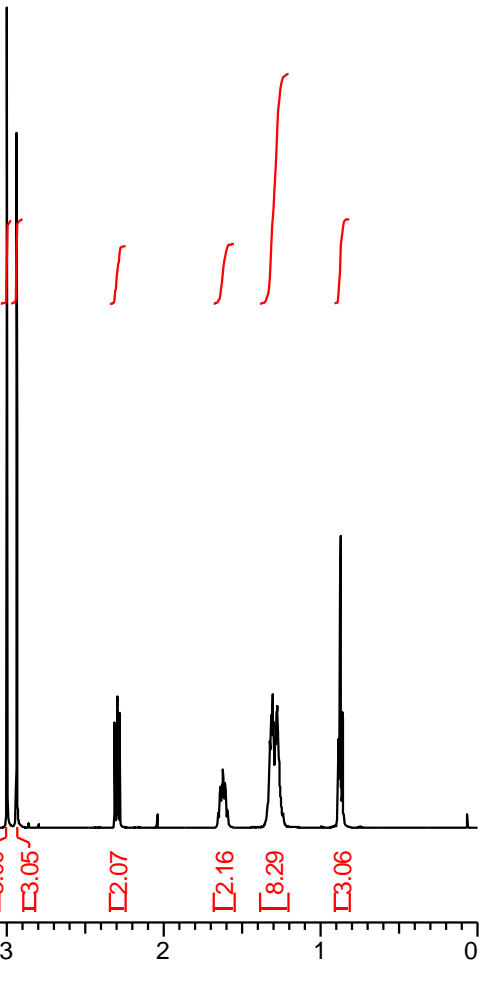

$\frac{\overline{7}}{\frac{\pi}{2}}$

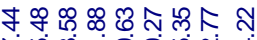

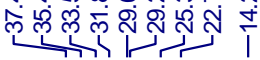

ND-522-5-SC.020.esp

Frequency (MHz): 125.76

Solvent: CHLOROFORM-d

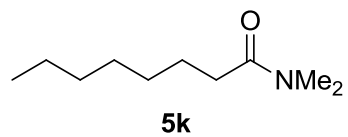

$5 k$ 
ND-522-4-SC.010.esp

Frequency (MHz): 500.13

Solvent: CHLOROFORM-d

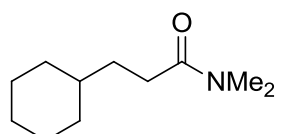

5 I

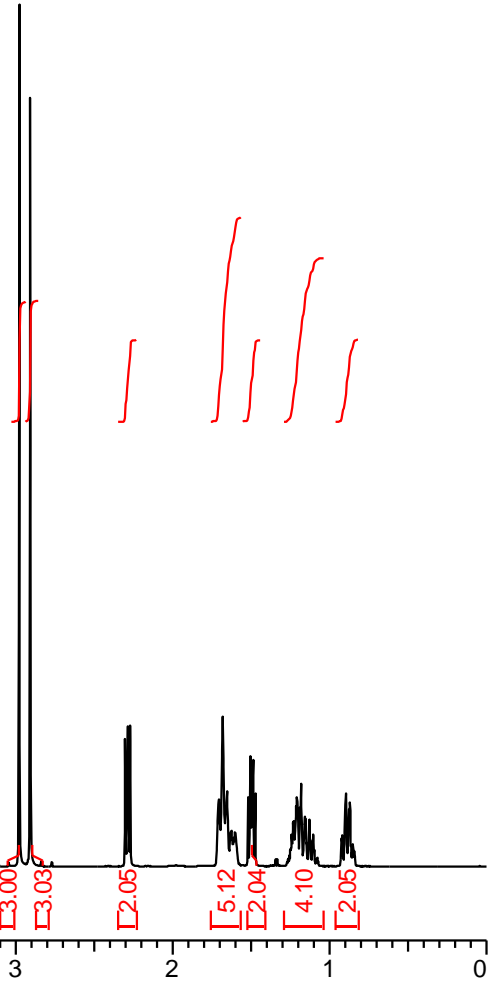

மึ భ

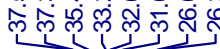

ND-522-4-SC.011.esp

Frequency (MHz): 125.76

Solvent: CHLOROFORM-d

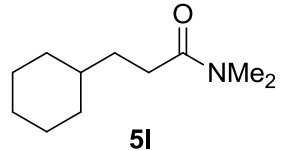

5

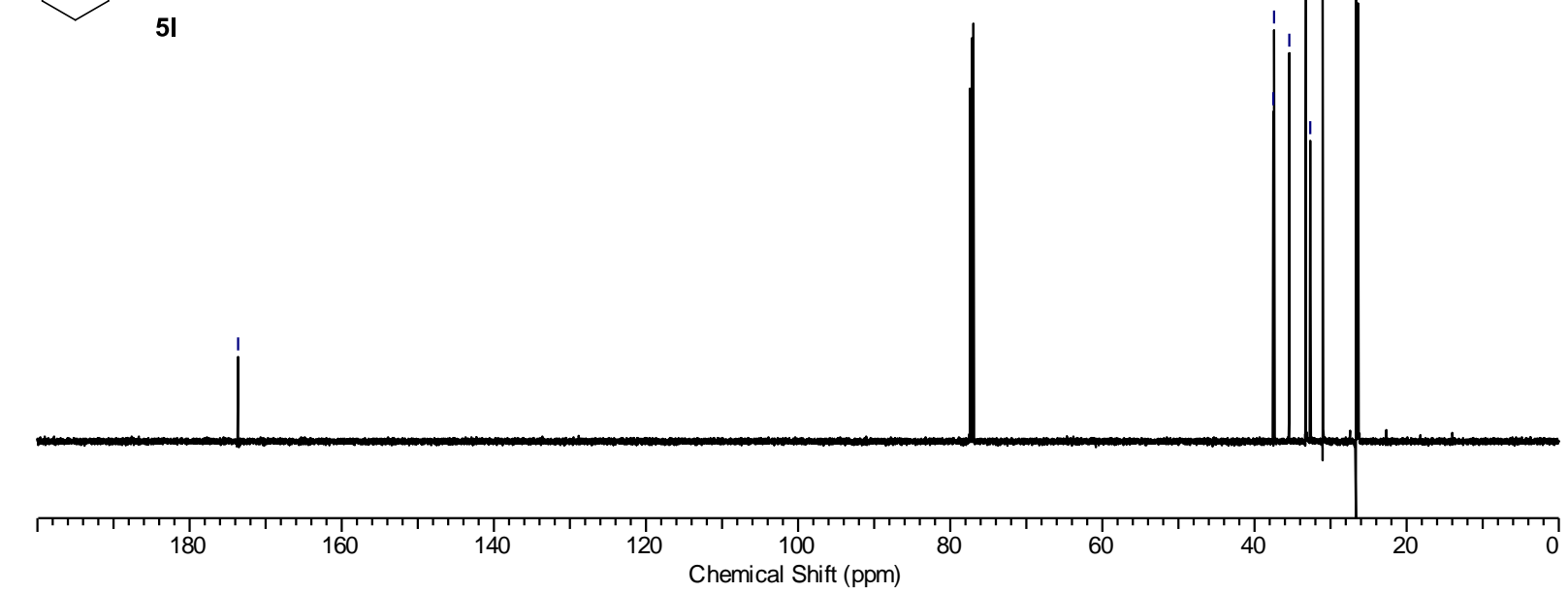


ND-537-7.010.esp

Frequency (MHz): 500.13

Solvent: CHLOROFORM-d

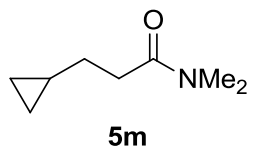

$5 \mathrm{~m}$

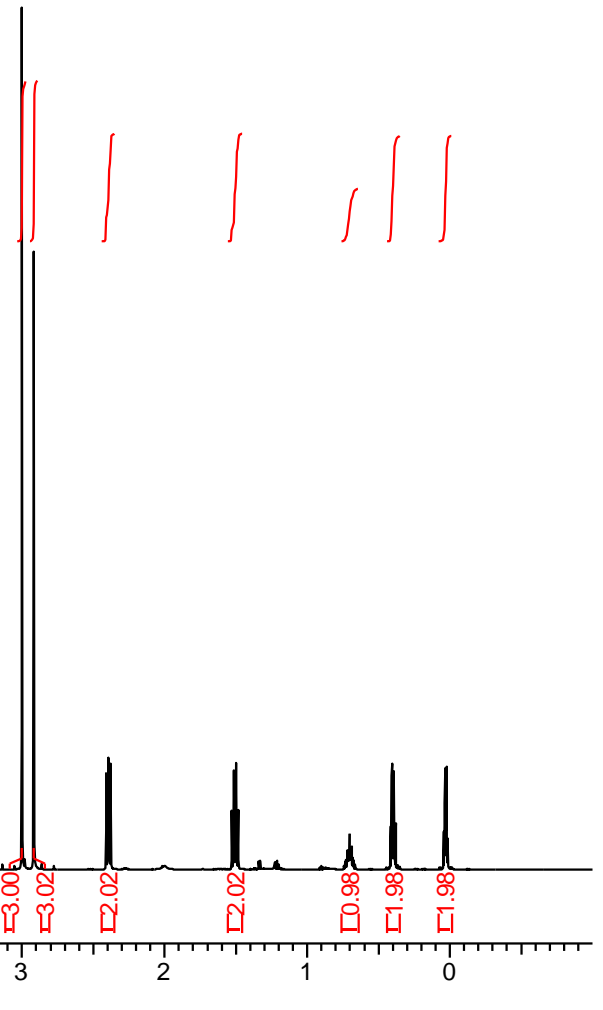

$\frac{\text { กิ }}{\frac{1}{1}}$

娄尔昌

लिल लू

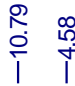

ND-537-7.011.esp

Frequency (MHz): 125.76

Solvent: CHLOROFORM-d

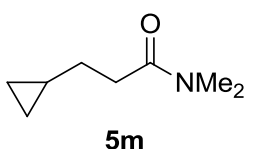

$5 m$

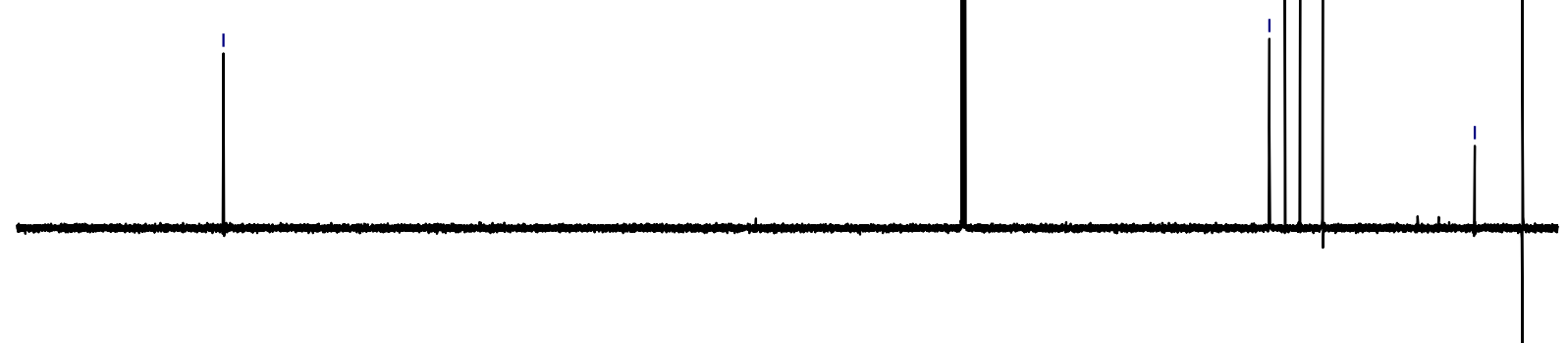

180

140

120

100

Chemical Shift (ppm)

80

60

40

20

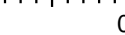


ND-523-4-SC.010.esp

Frequency $(\mathrm{MHz}): 500.13$

Solvent: CHLOROFORM-d

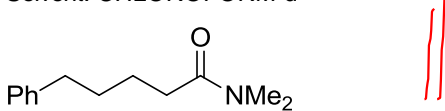

$5 n$
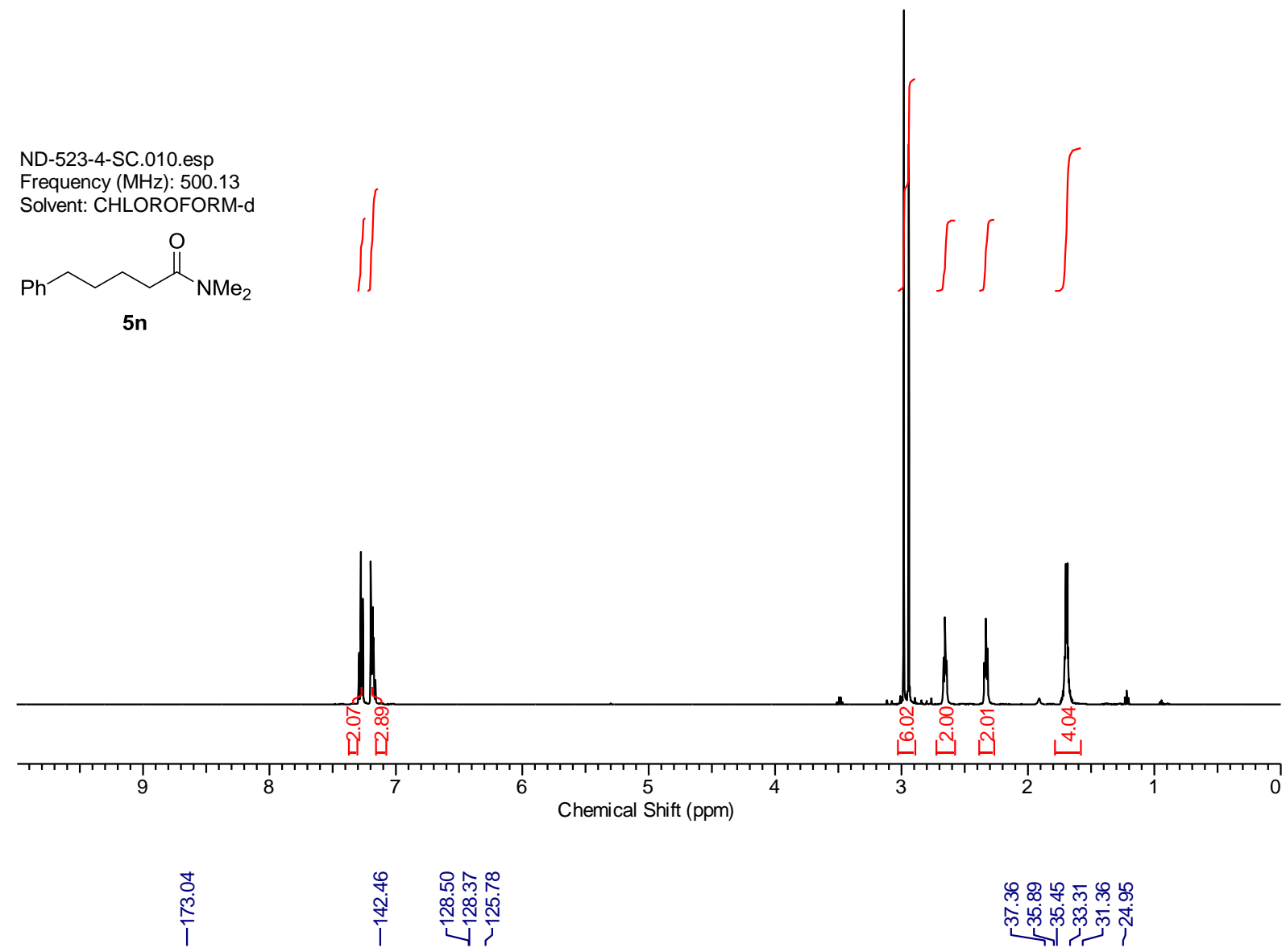

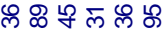

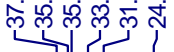

ND-523-4-SC.011.esp

Frequency (MHz): 125.76

Solvent: CHLOROFORM-d

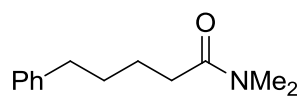

$5 n$

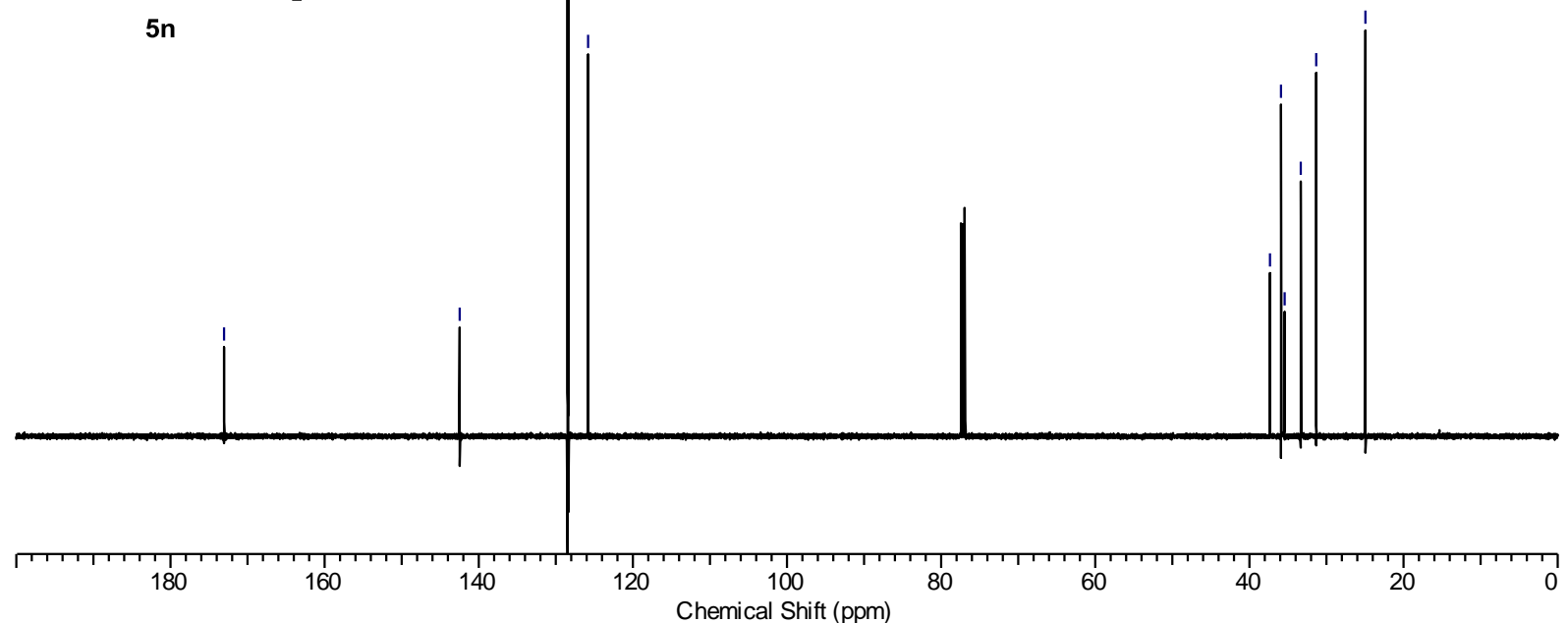


ND-638-1-sc.010.esp

Frequency (MHz): 500.13

Solvent: CHLOROFORM-d

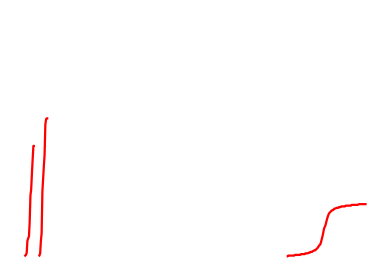

$\overbrace{50}^{\mathrm{H}^{-M e}}$
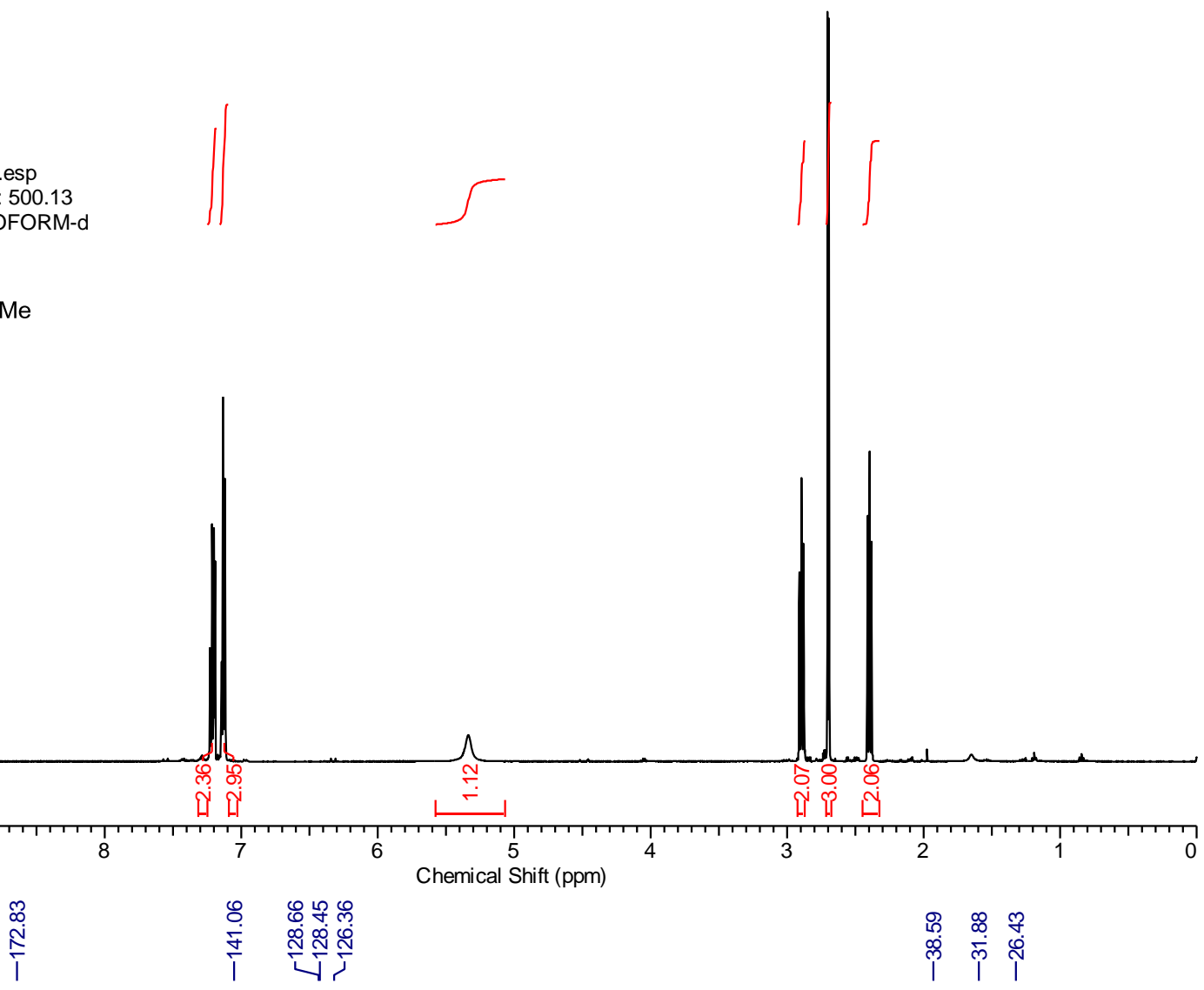

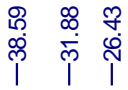

ND-638-1-sc.011.esp

Frequency (MHz): 125.76

Solvent: CHLOROFORM-d

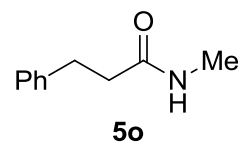

然

5
Chemical Shift (ppm)

ㄴำ

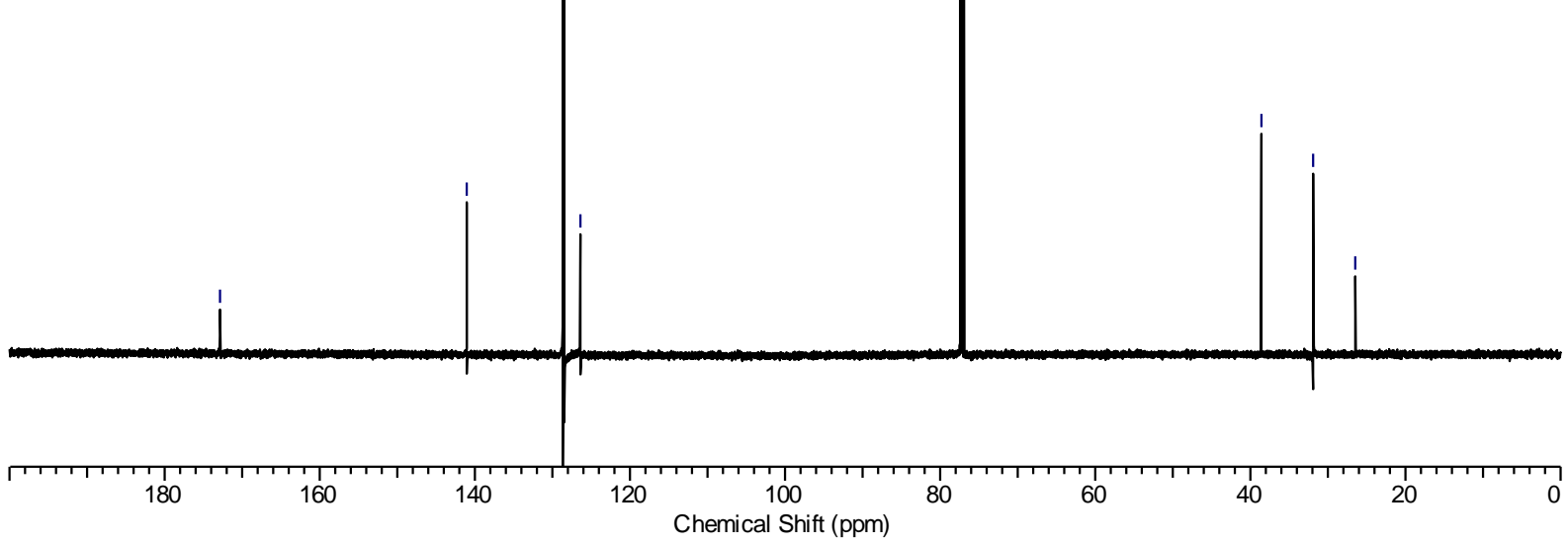




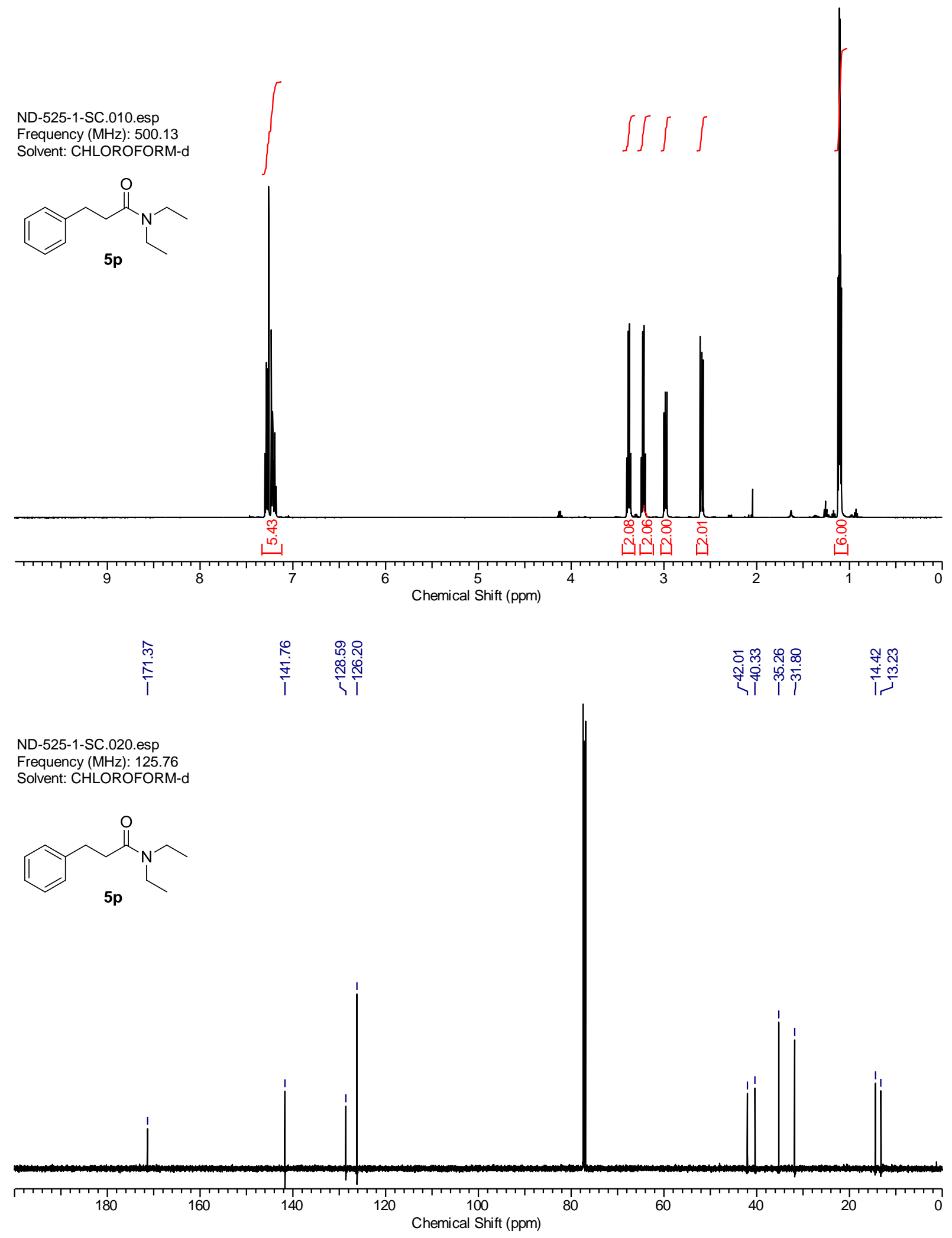




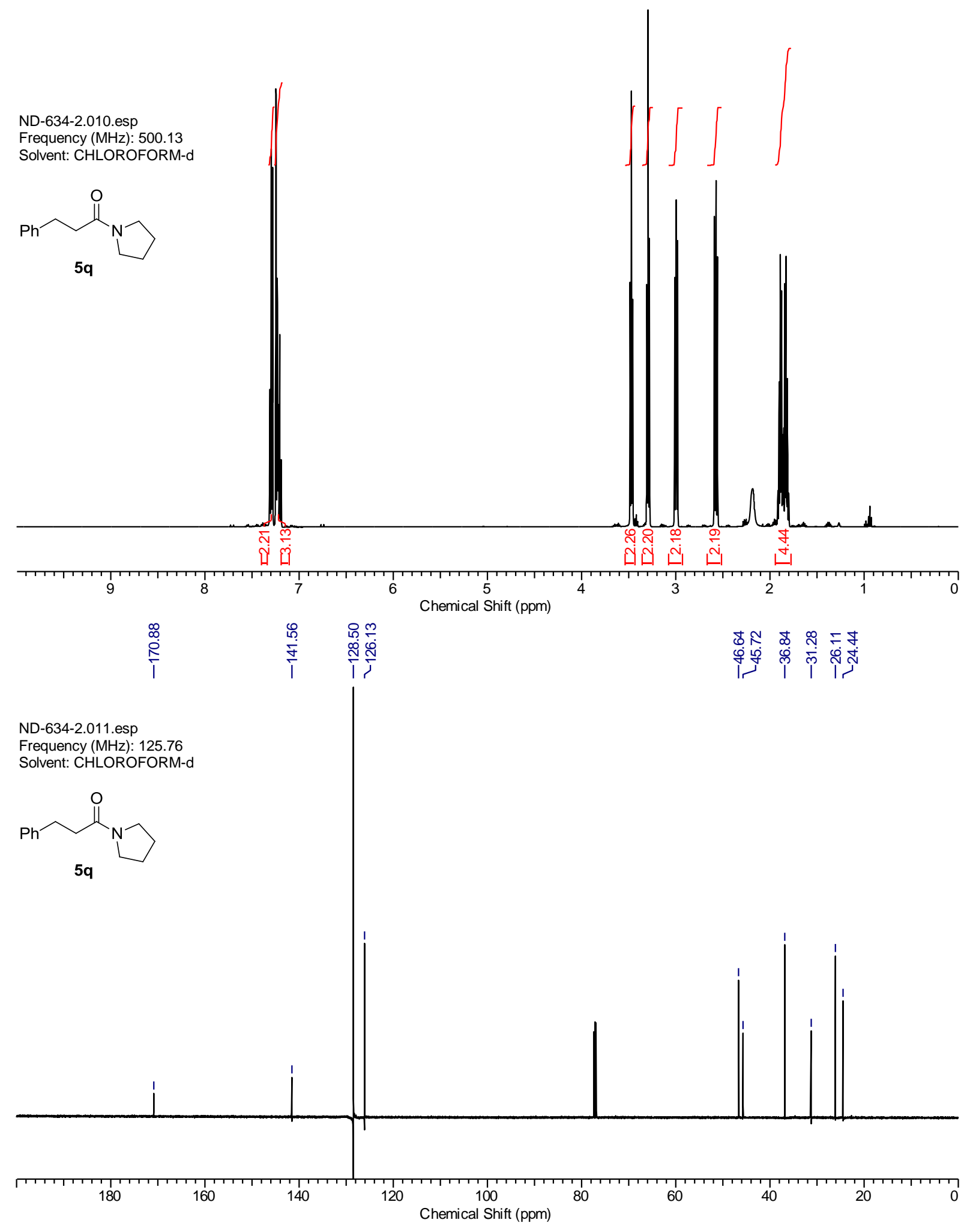




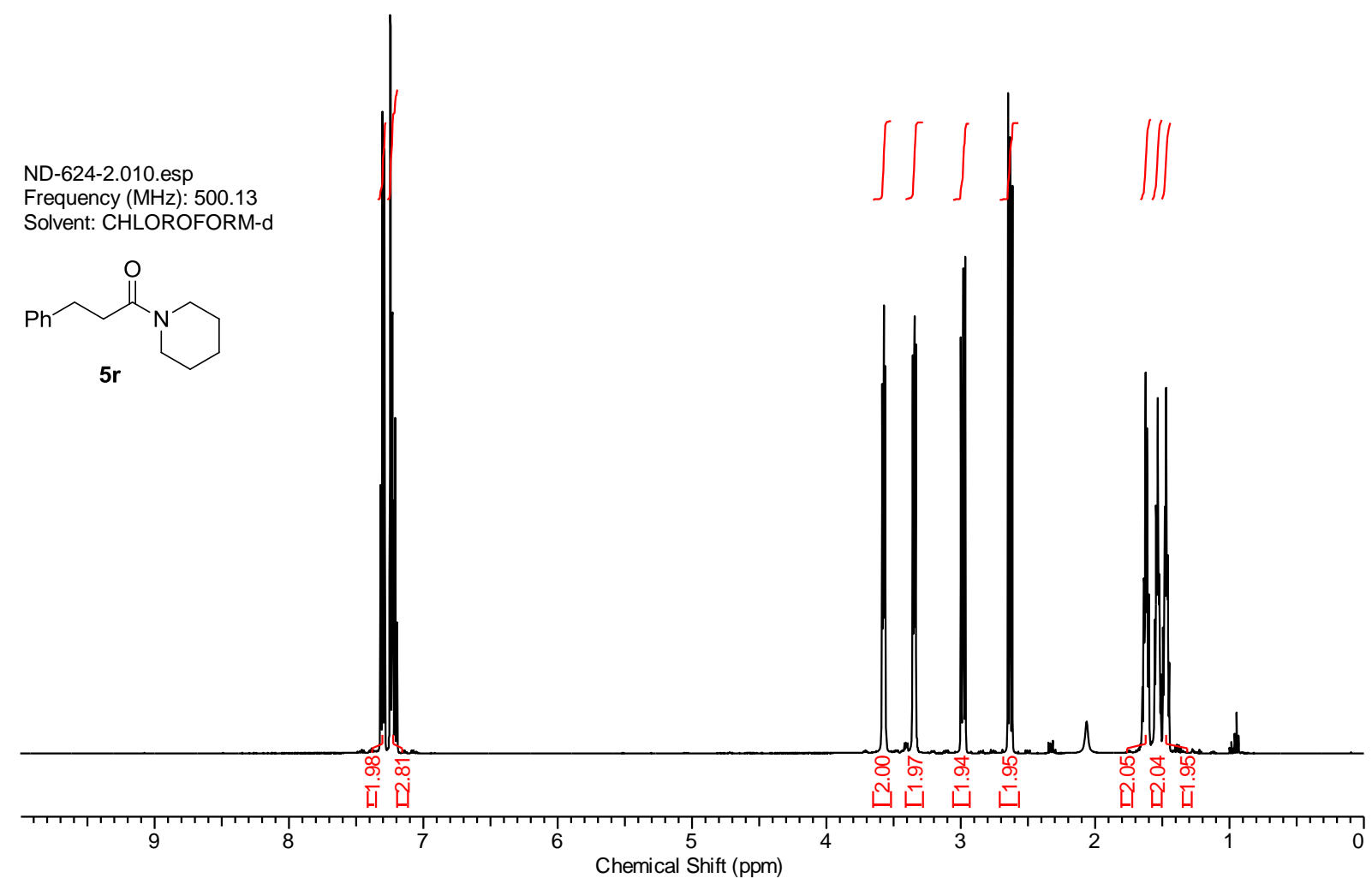

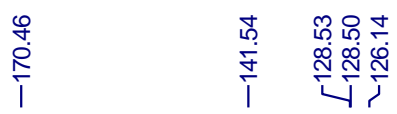

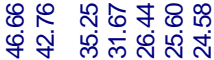

।

ND-624-2.011.esp

Frequency (MHz): 125.76

Solvent: CHLOROFORM-d<smiles>O=C(CCc1ccccc1)N1CCCCC1</smiles>

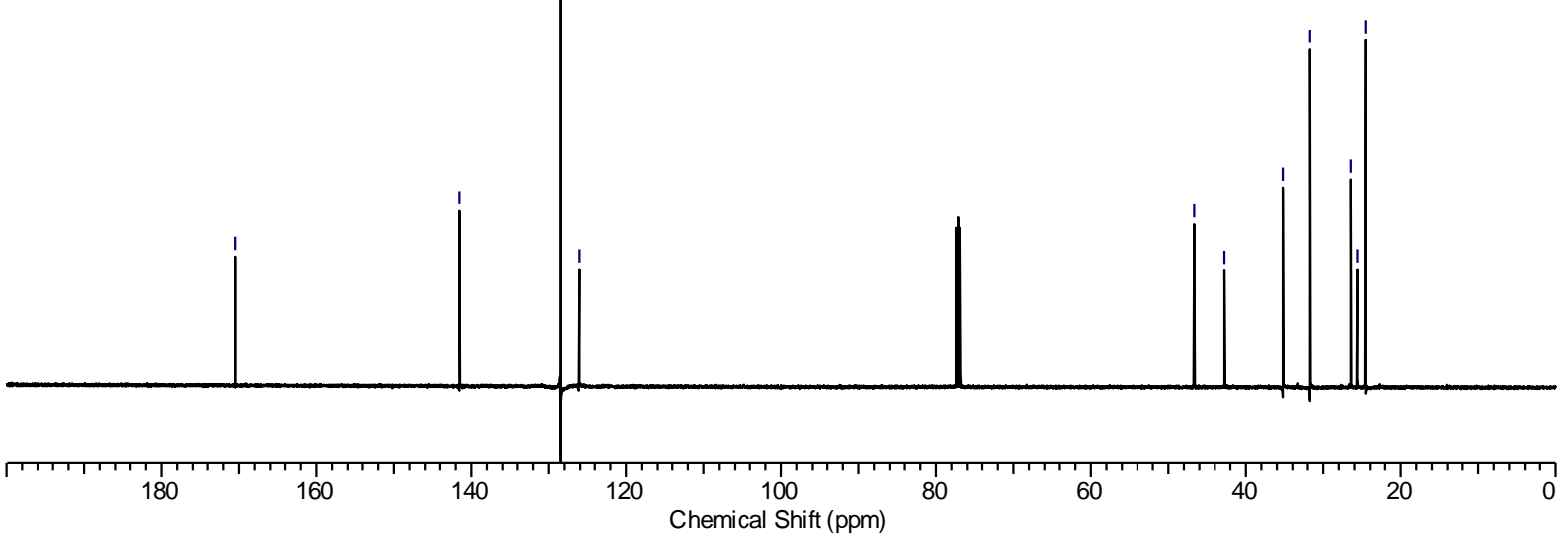



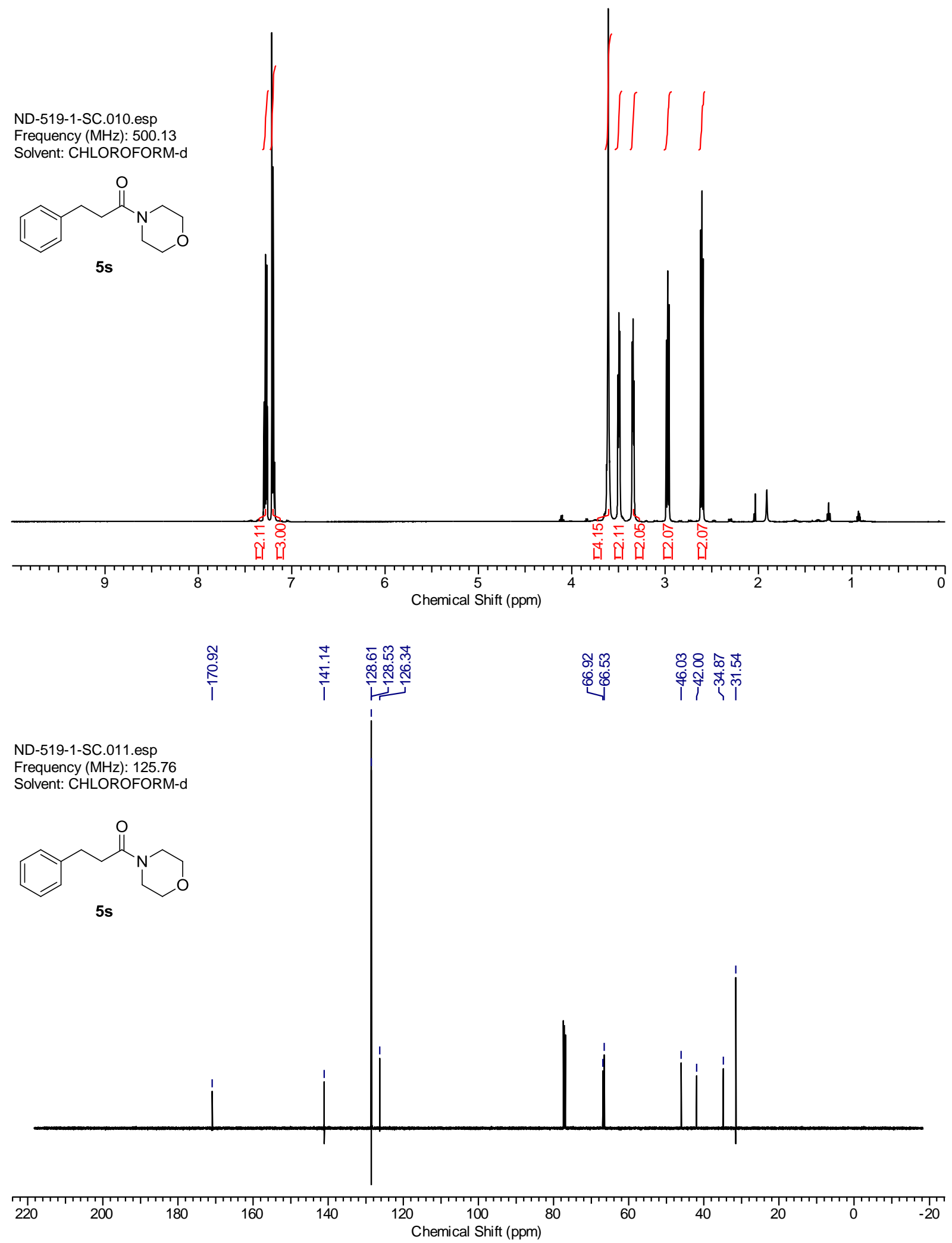
<smiles>COc1ccc(CCC(=O)N2CCOCC2)cc1</smiles>

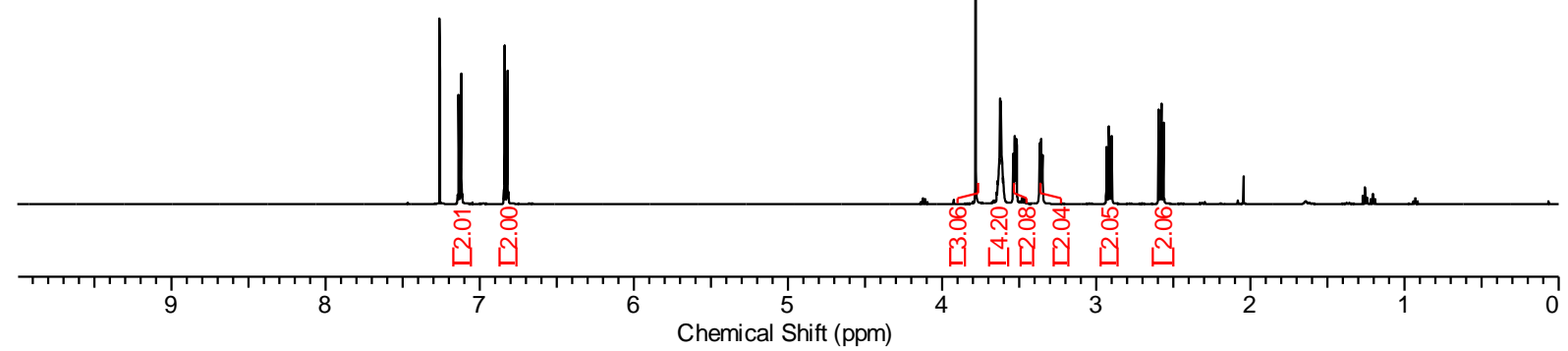

\begin{tabular}{|c|c|c|c|c|c|c|}
\hline 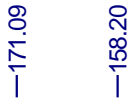 & 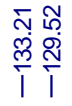 & $\frac{\stackrel{L}{0}}{\underset{+}{\leftarrow}}$ & 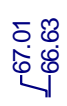 & † & 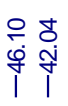 & 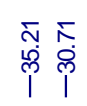 \\
\hline
\end{tabular}

ND-544-1.011.esp

Frequency (MHz): 125.76

Solvent: CHLOROFORM-d<smiles>COc1ccc(CCC(=O)N2CCOCC2)cc1</smiles>

$5 t$

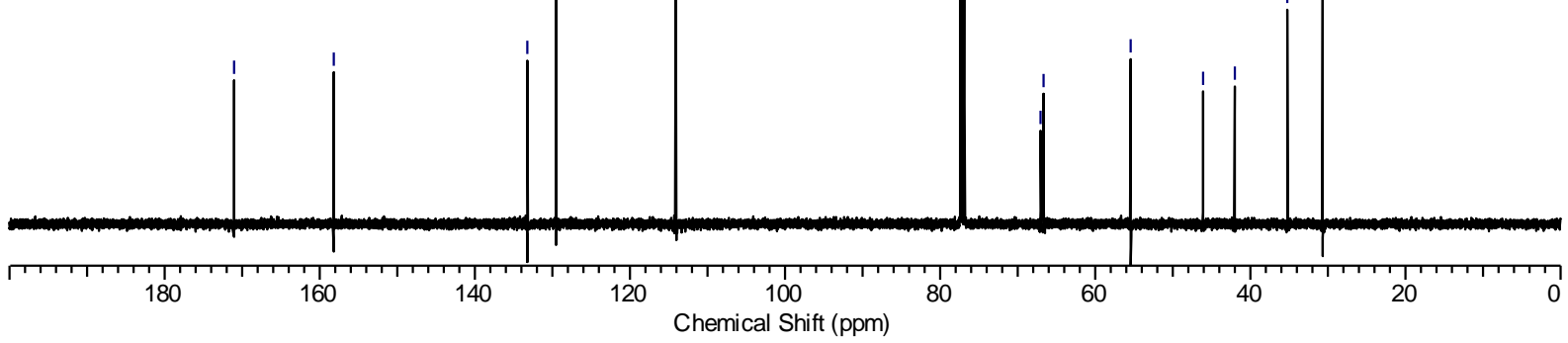


ND-598.012.esp

Frequency (MHz): 500.13

Solvent: CHLOROFORM-d
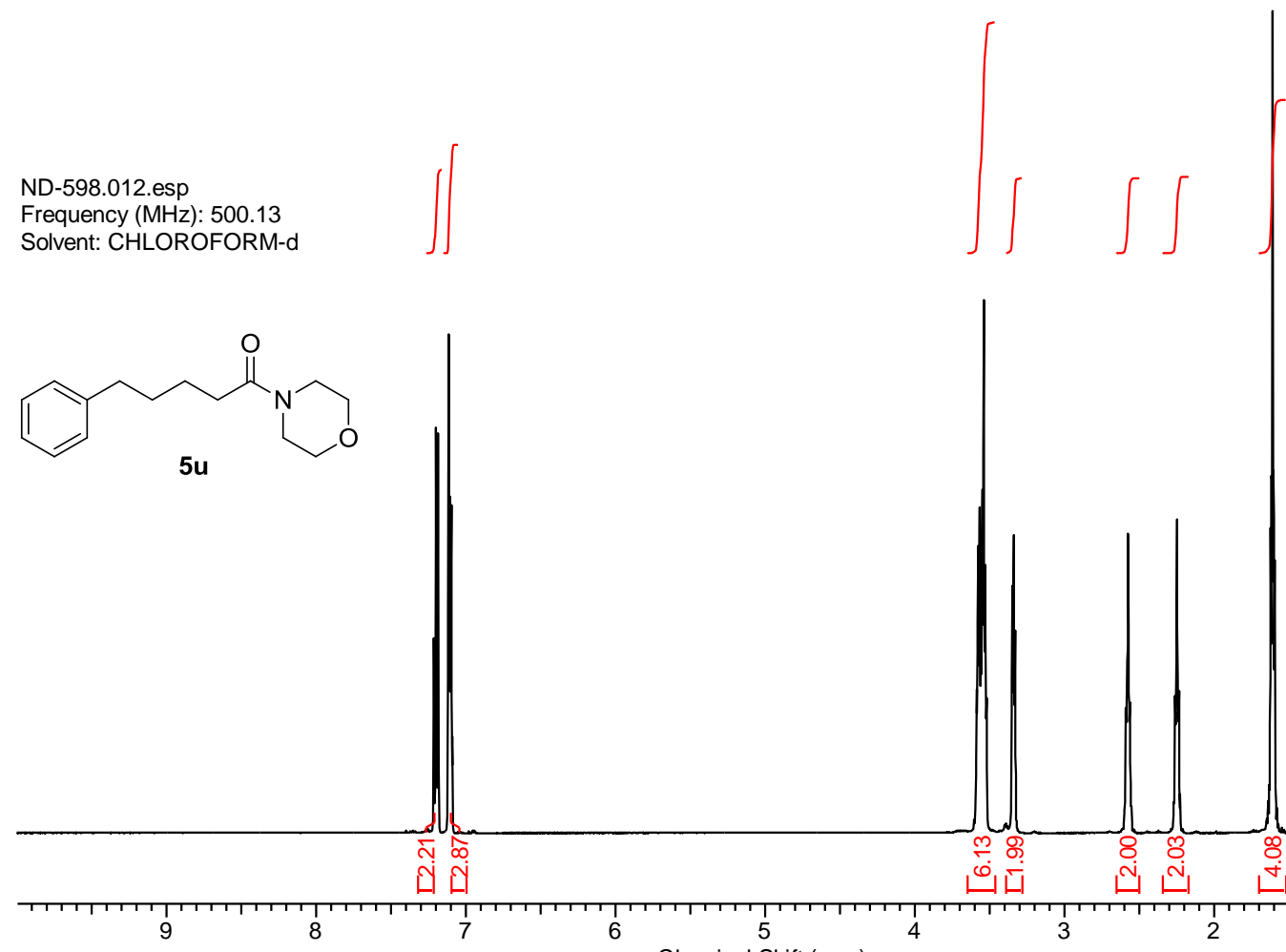

m. ம) ํ.

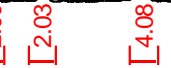

Chemical Shift (ppm)
$\frac{\hat{\sigma}}{\frac{1}{1}}$

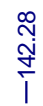

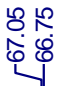
으 \& 옹ำ

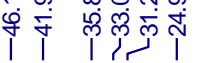

ND-598.013.esp

Frequency (MHz): 125.76

Solvent: CHLOROFORM-d<smiles>O=C(CCCCc1ccccc1)N1CCOCC1</smiles>

$5 u$
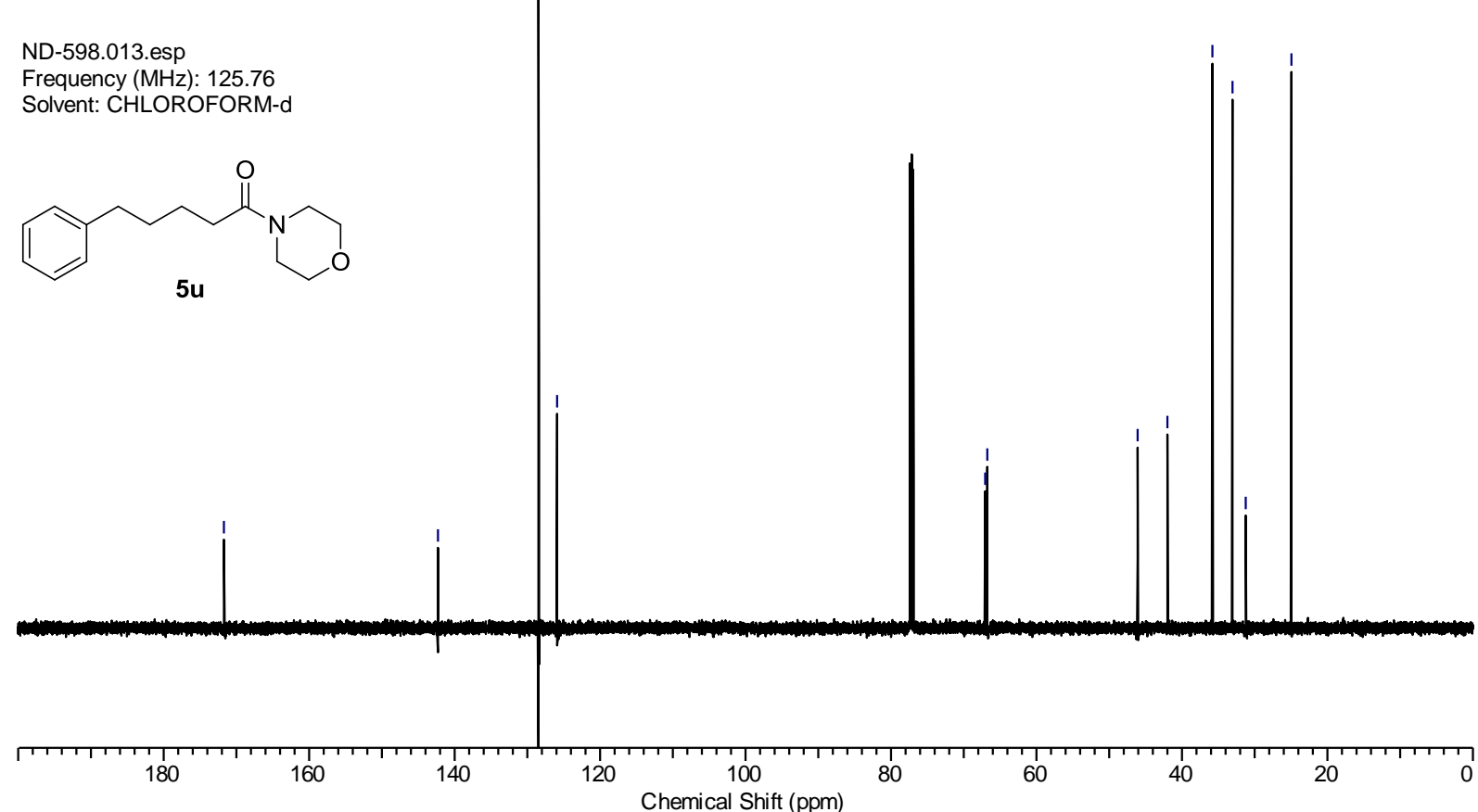
ND-544-2.010.esp

Frequency $(\mathrm{MHz}): 500.13$

Solvent: CHLOROFORM-d<smiles>O=C(CCC1CC1)N1CCOCC1</smiles>

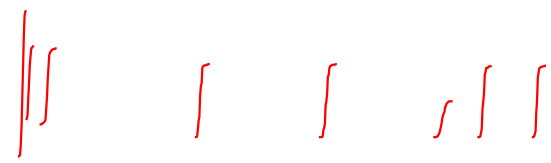

सก โ

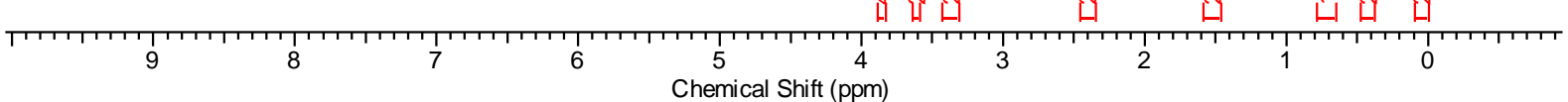

\section{$\frac{\infty}{\frac{\infty}{1}}$}

\section{무}

仓ैं

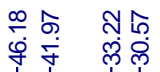

임 움

ND-544-2.011.esp

Frequency (MHz): 125.76

Solvent: CHLOROFORM-d<smiles>O=C(CCC1CC1)N1CCOCC1</smiles>

5v

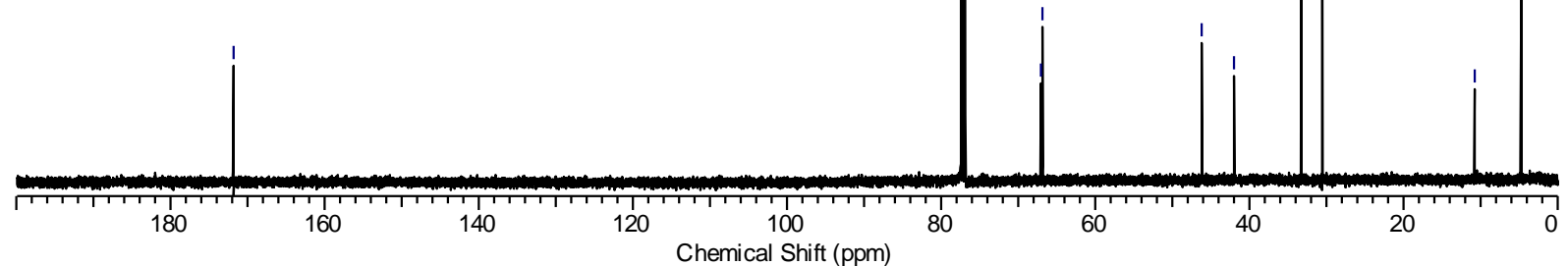


Ester-Alkylation

ND-527-2.010.esp

Frequency (MHz): 500.13

Solvent: CHLOROFORM-d<smiles>CC(C)(C)OC(=O)CCc1ccccc1</smiles>

$6 a$

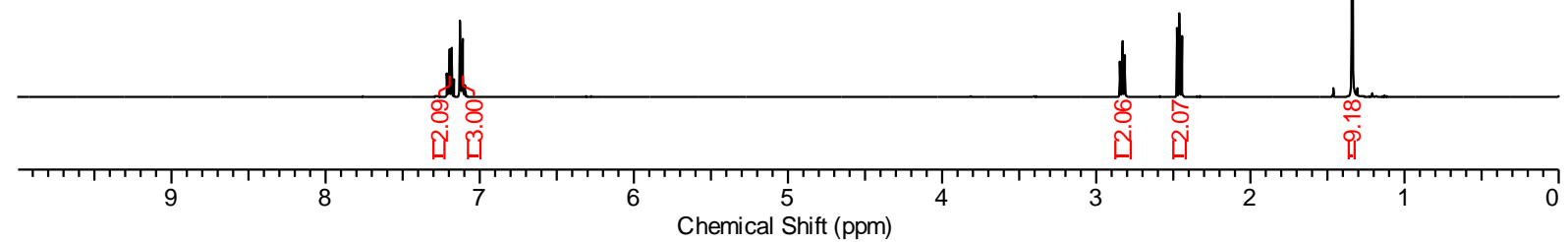

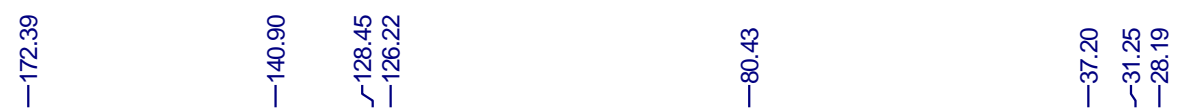

ND-527-2.011.esp

Frequency (MHz): 125.76

Solvent: CHLOROFORM-d<smiles>CC(C)(C)OC(=O)CCc1ccccc1</smiles>

$6 a$

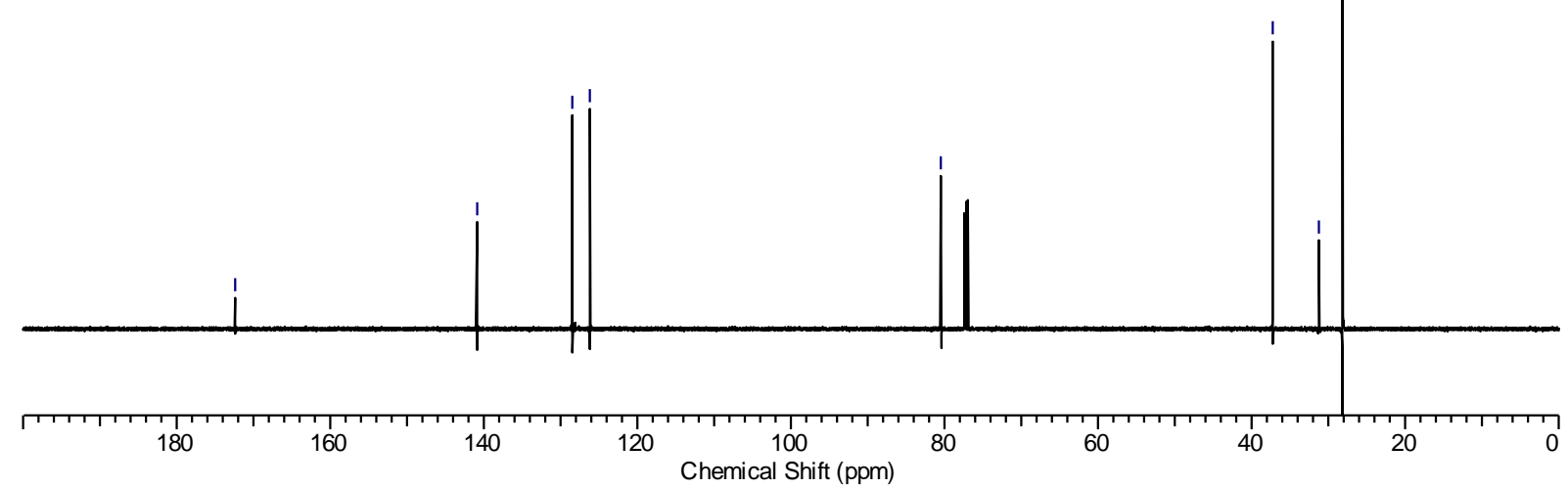


ND-532-4.010.esp

Frequency (MHz): 500.13

Solvent: CHLOROFORM-d

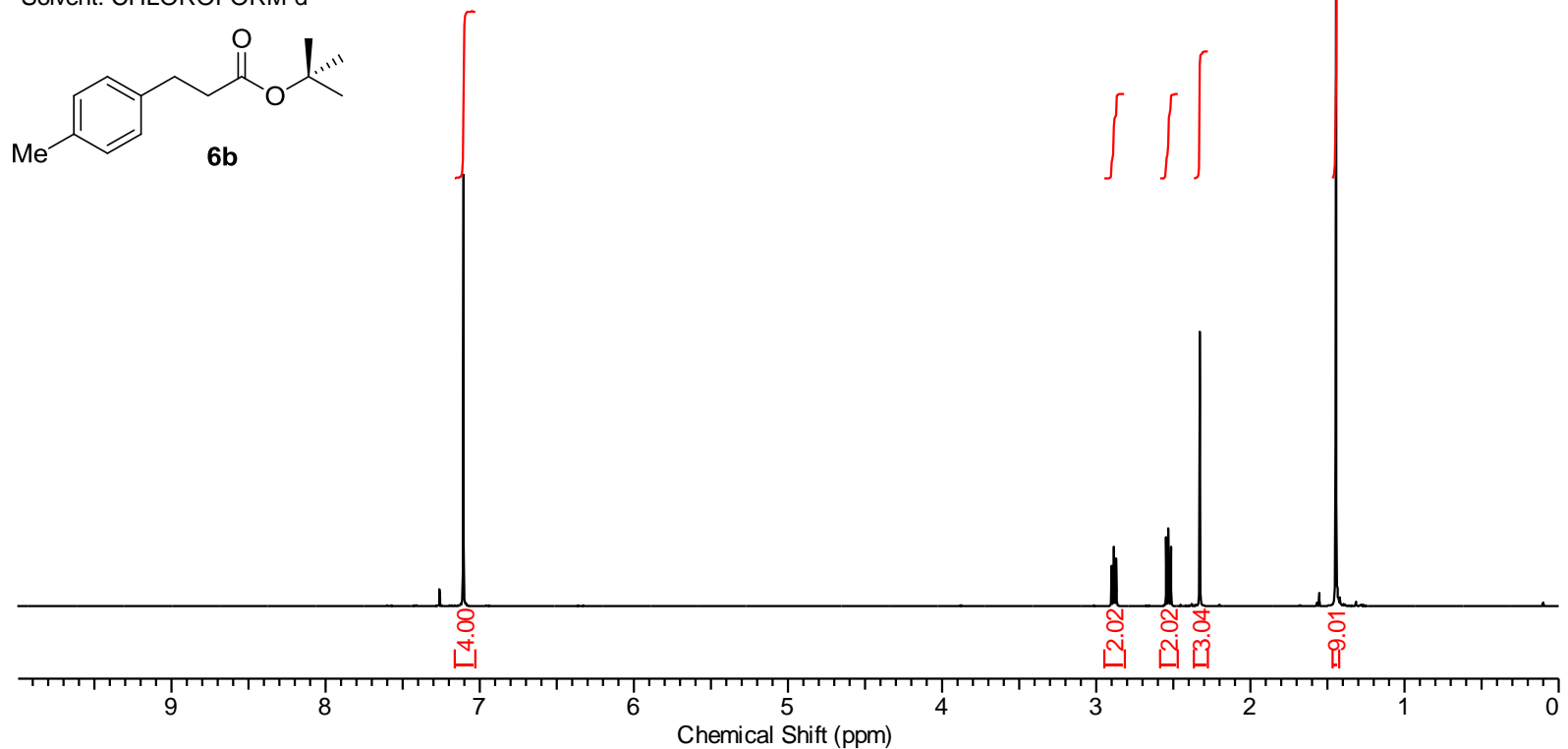

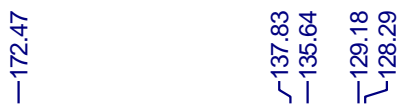

@

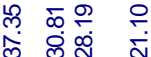

ND-532-4.011.esp

Frequency (MHz): 125.76

Solvent: CHLOROFORM-d<smiles>Cc1ccc(CCC(=O)OC(C)(C)C)cc1</smiles>

$6 b$

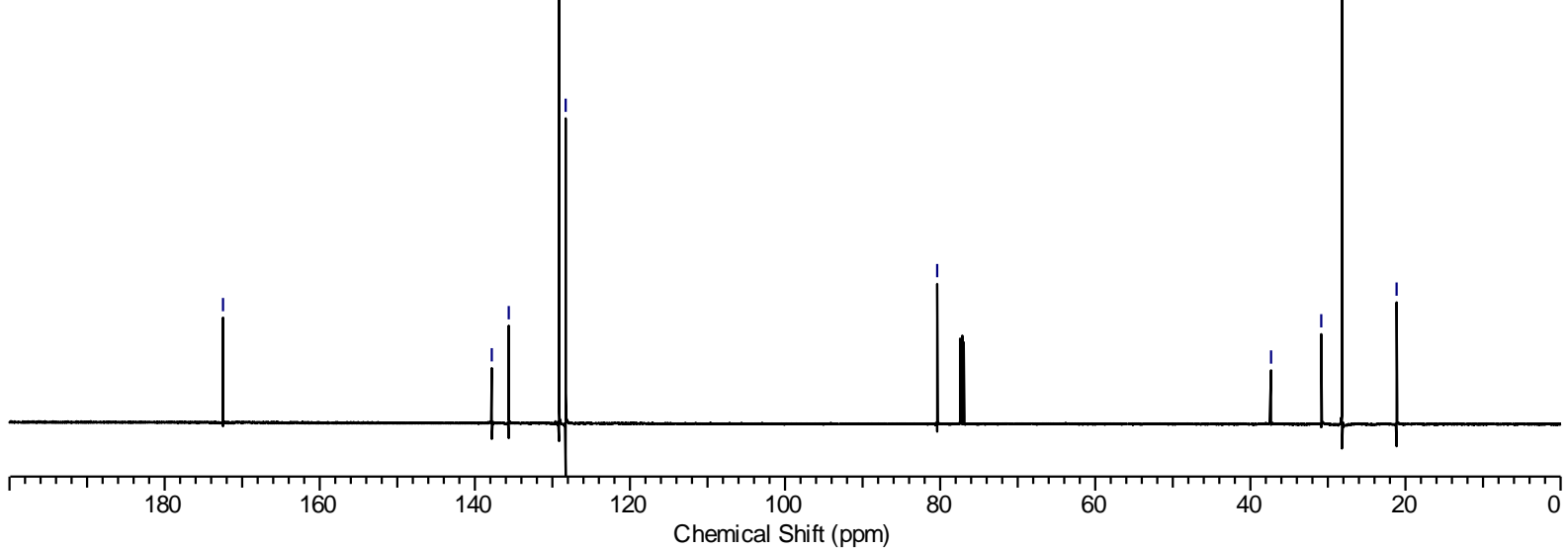


ND-532-6.010.esp

Frequency (MHz): 500.13

Solvent: CHLOROFORM-d
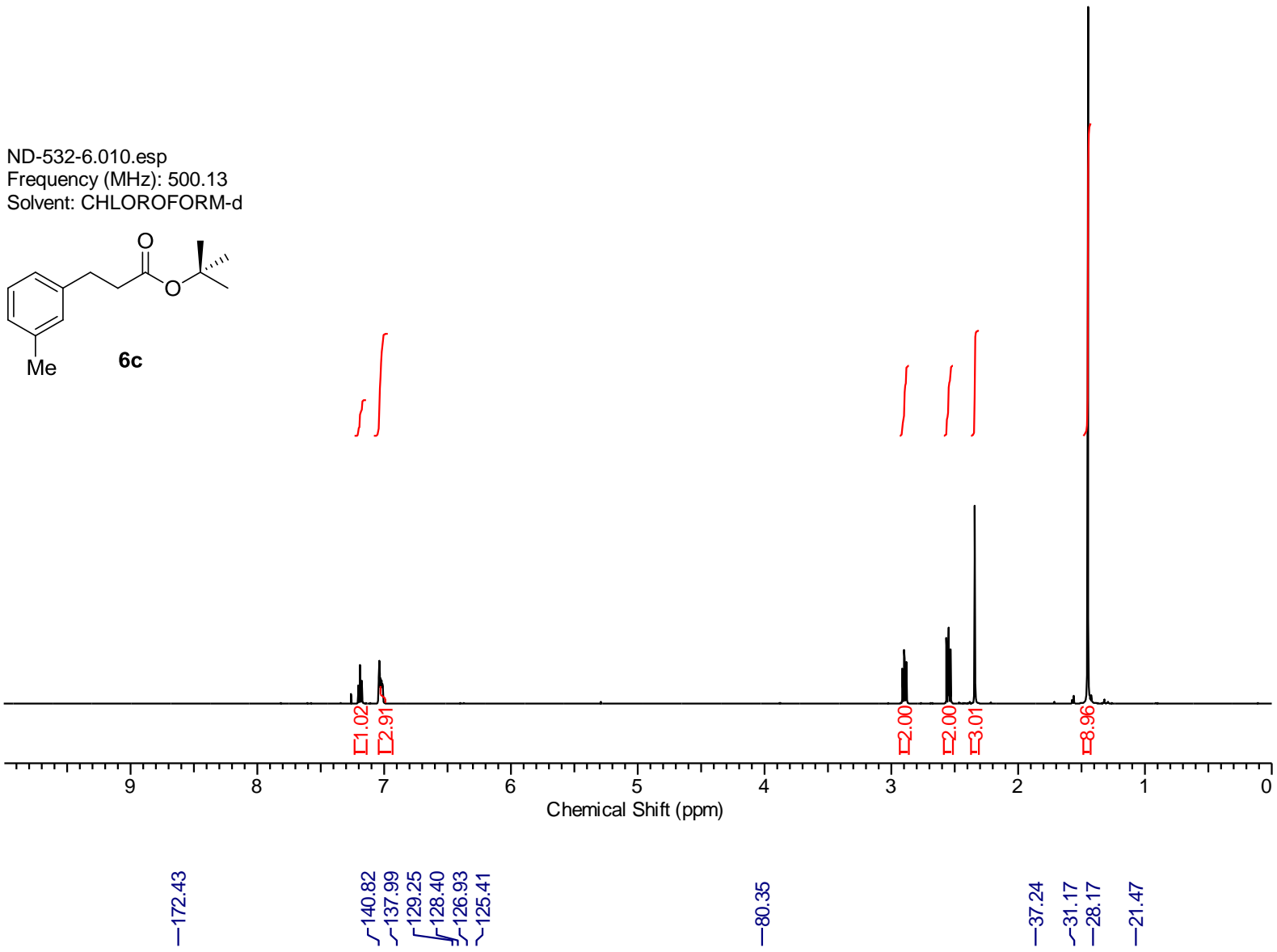

ND-532-6.011.esp

Frequency (MHz): 125.76

Solvent: CHLOROFORM-d<smiles>Cc1cccc(CCC(=O)OC(C)(C)C)c1</smiles>

Me 6c

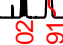

元藏

Chemical Shift (ppm)

ले क्ञ

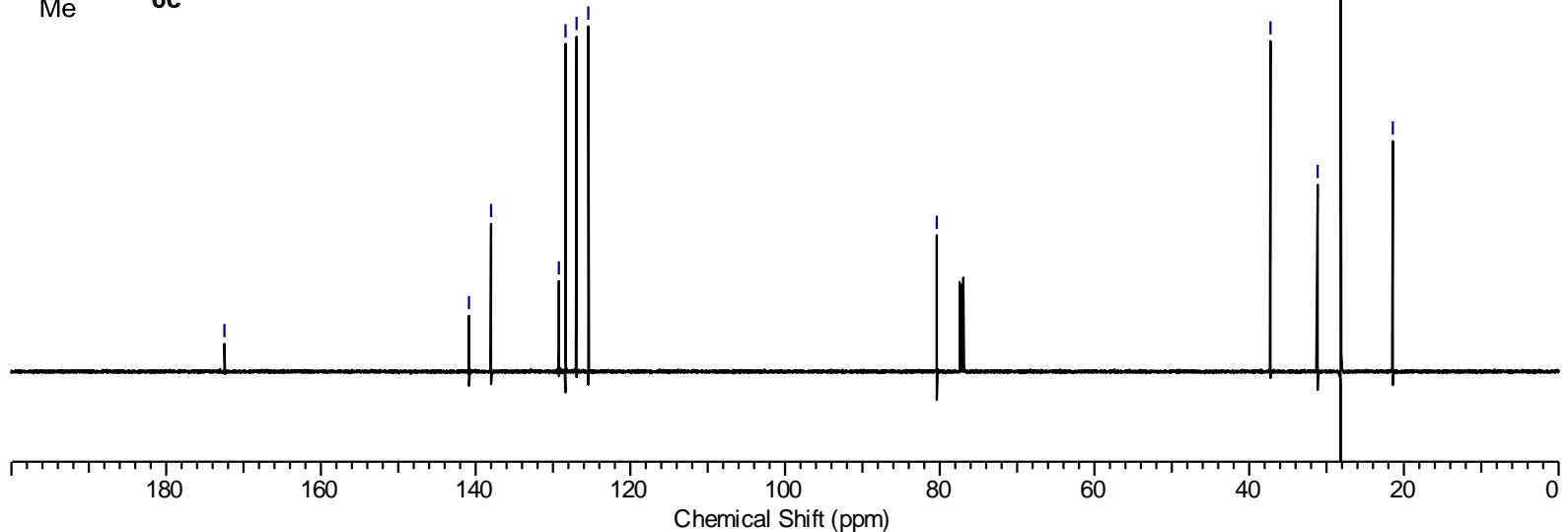


ND-534-4.010.esp

Frequency (MHz): 500.13

Solvent: CHLOROFORM-d
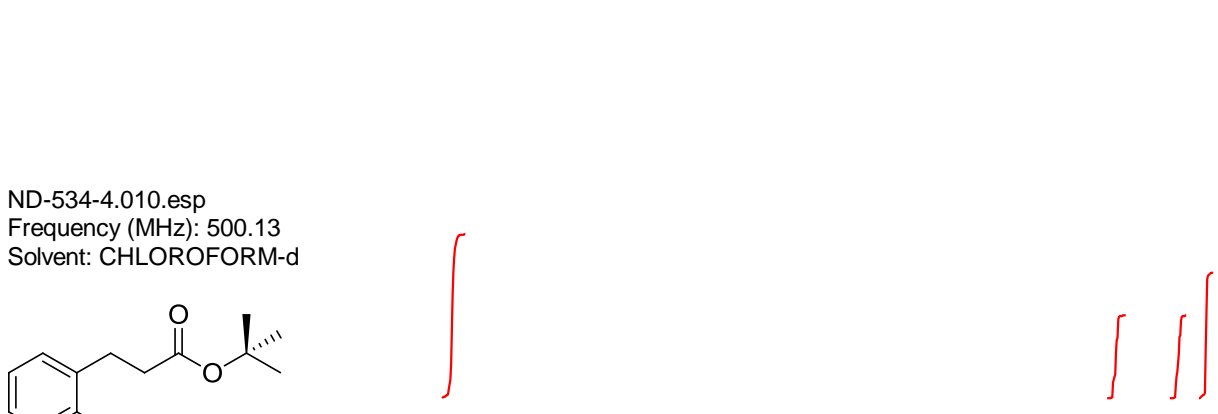

6d
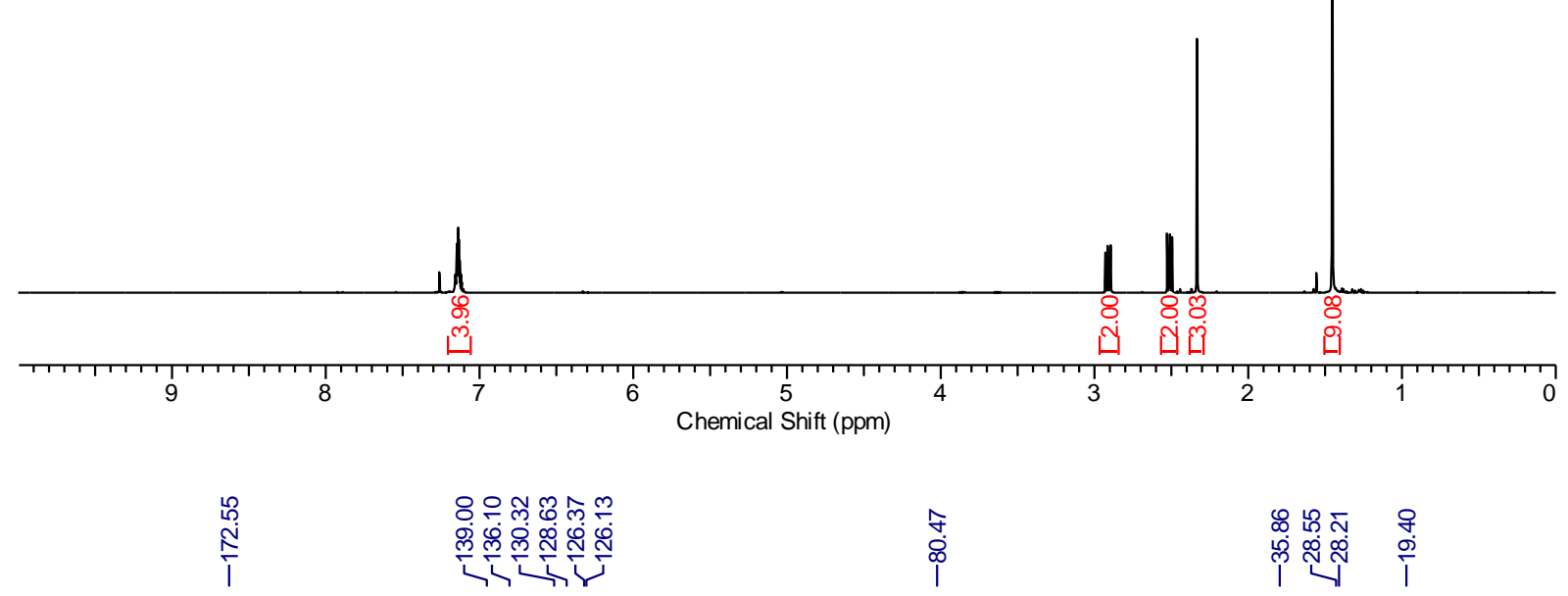

年

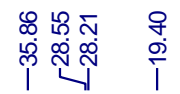

ND-534-4.011.esp

Frequency (MHz): 125.76

Solvent: CHLOROFORM-d<smiles>Cc1ccccc1CCC(=O)OC(C)(C)C</smiles>

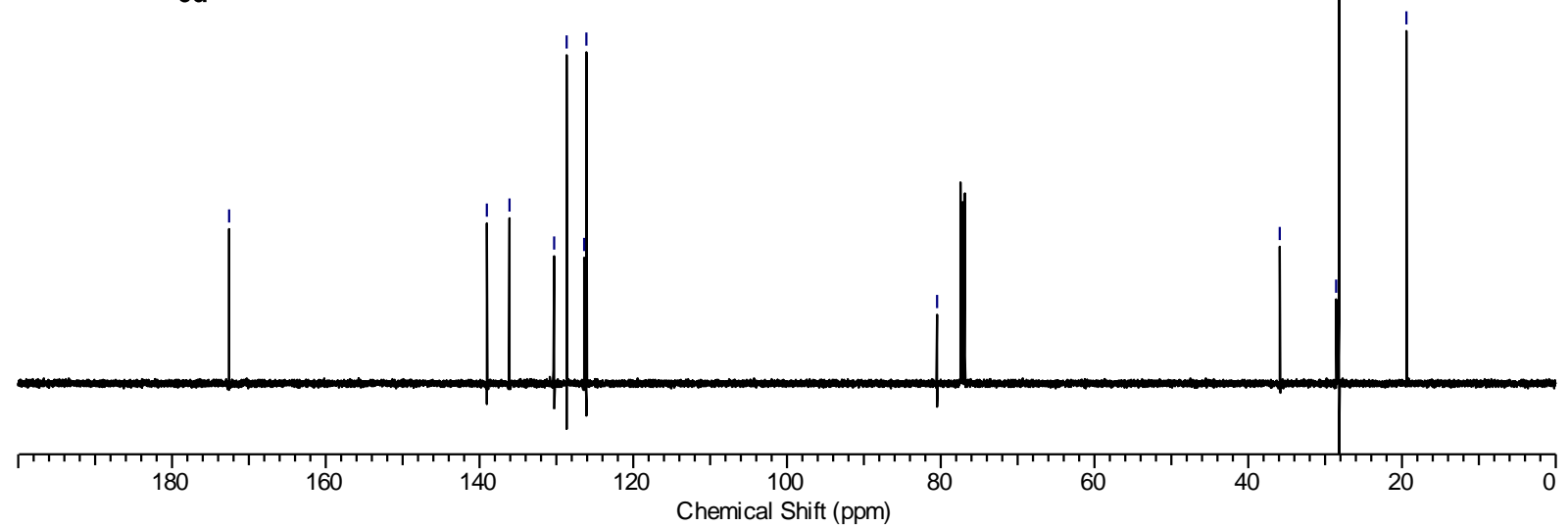


ND-590-7.010.esp

Frequency (MHz): 500.13

Solvent: CHLOROFORM-d
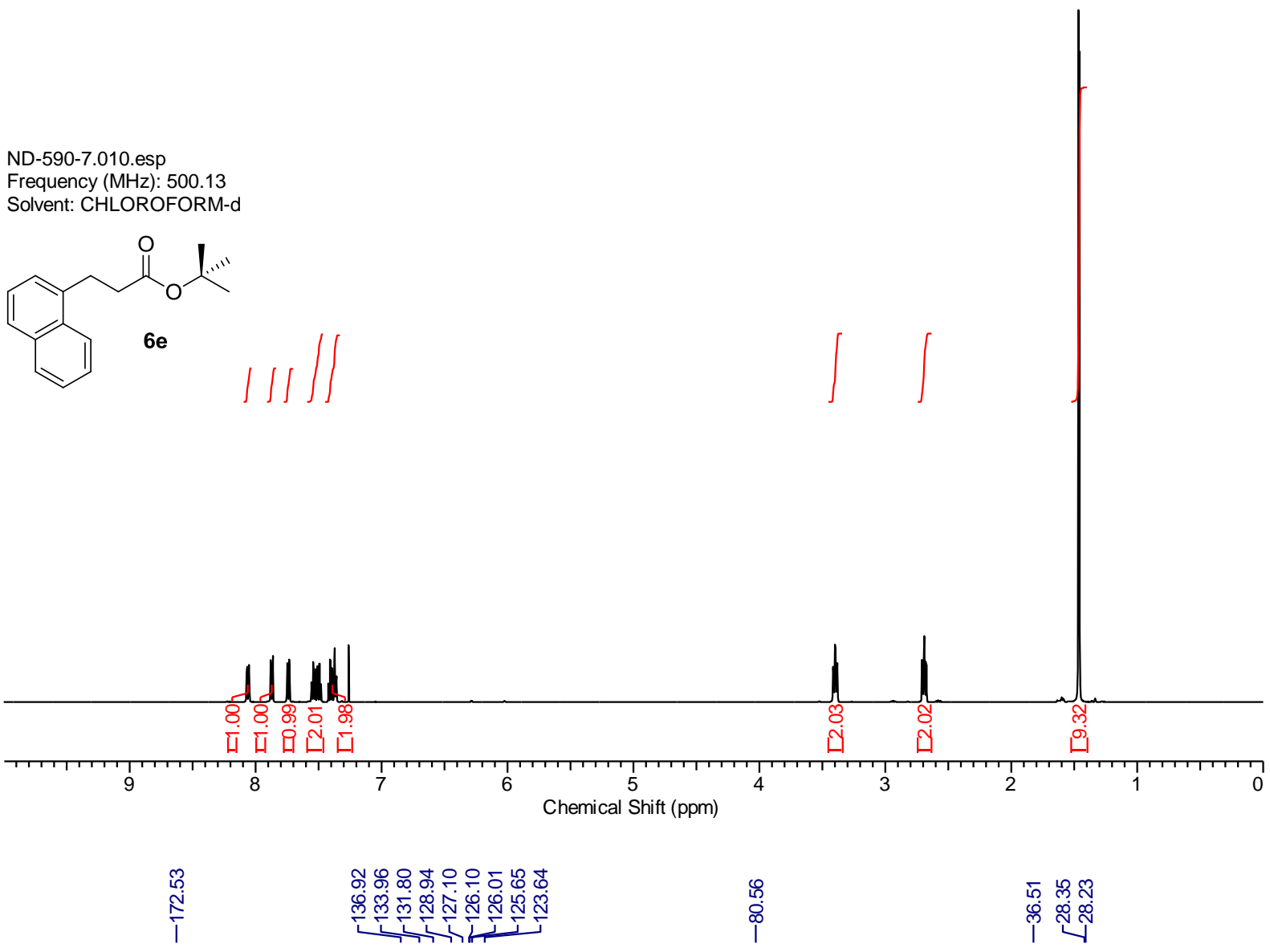

ND-590-7.011.esp

Frequency (MHz): 125.76

Solvent: CHLOROFORM-d<smiles>CC(C)(C)OC(=O)CCc1cccc2ccccc12</smiles>

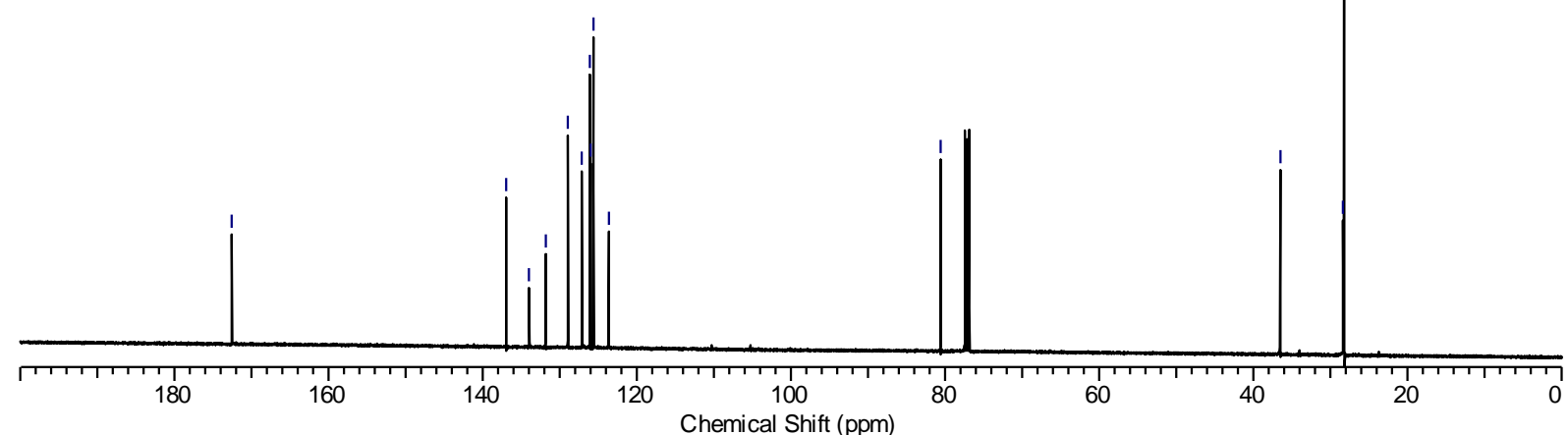


ND-508-1-SC_PROTON_20151015_01

Frequency $(\mathrm{MHz}): 299.86$

Solvent: CHLOROFORM-d<smiles>COc1ccc(CCC(=O)OC(C)(C)C)cc1</smiles><smiles>C1CCCCC1</smiles>

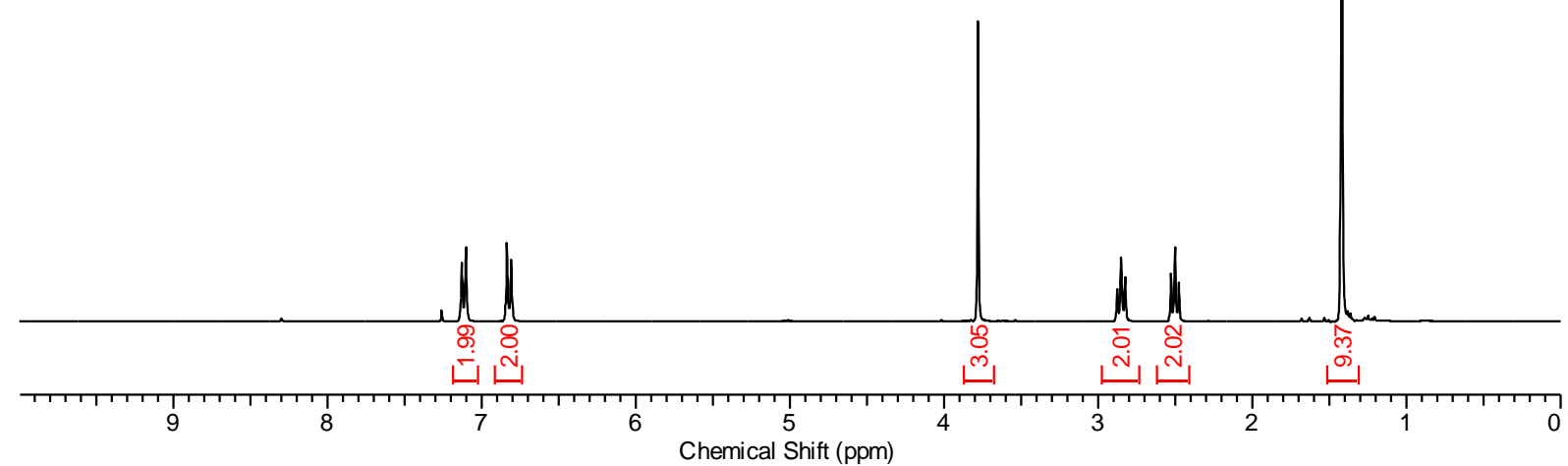

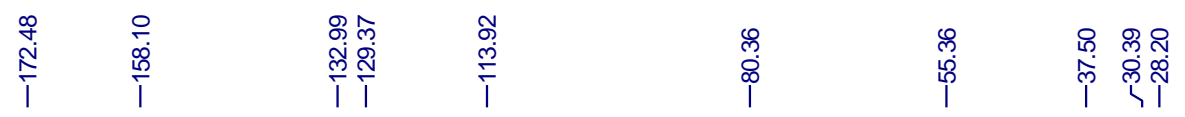

ND-508-1-SC_CARBON_20151015_01

Frequency (MHz): 75.41

Solvent: CHLOROFORM-d<smiles>COc1ccc(CCC(=O)OC(C)(C)C)cc1</smiles>

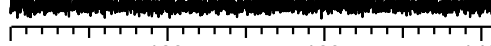

180 

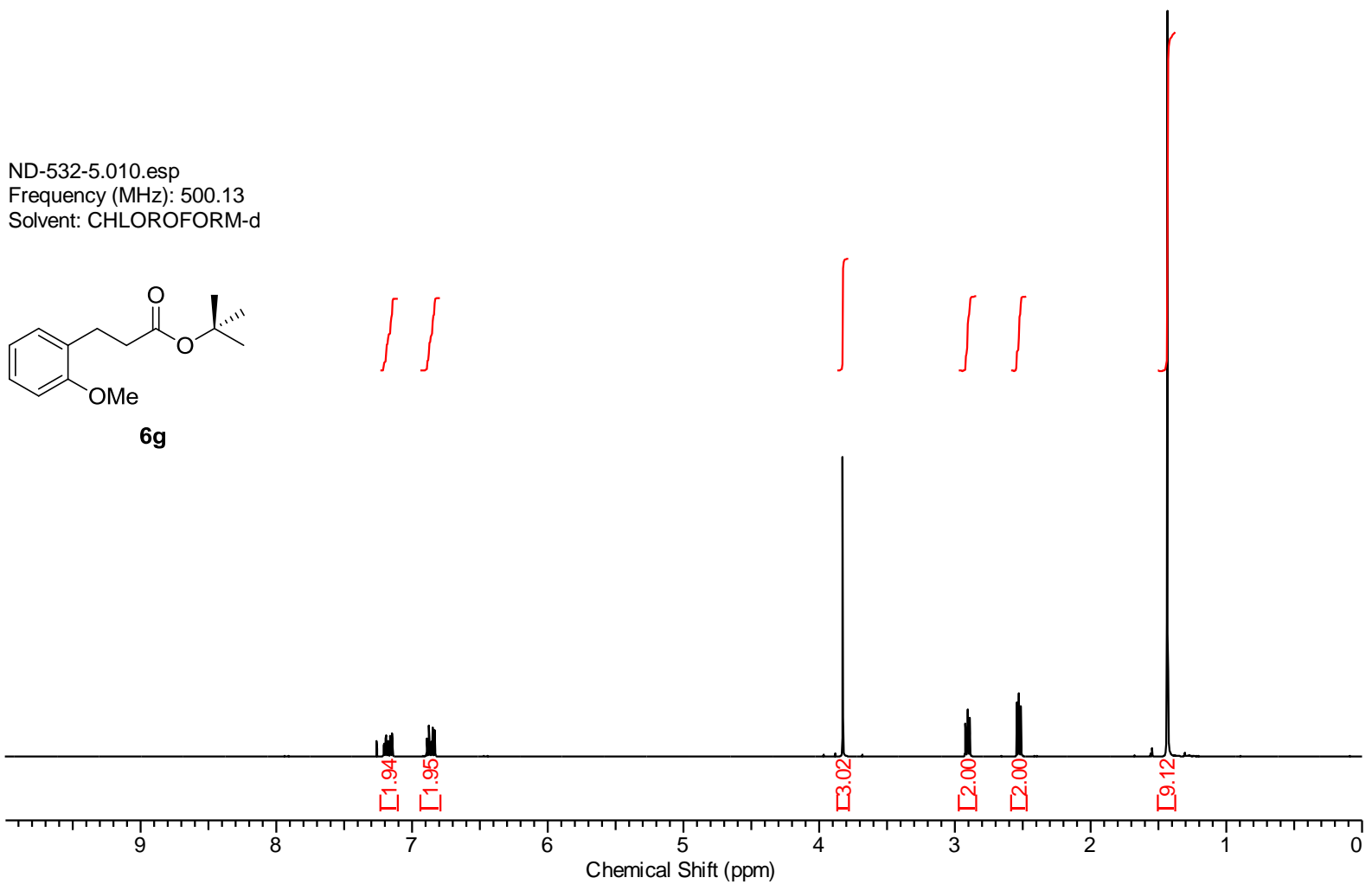

\begin{tabular}{|c|c|c|}
\hline $\begin{array}{l}\infty \\
\infty \\
\\
\end{array}$ & $\begin{array}{l}\text { ஸै } \\
\frac{10}{1} \\
1\end{array}$ & 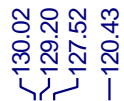 \\
\hline
\end{tabular}

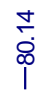

กิก

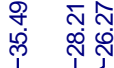

ND-532-5.011.esp

Frequency (MHz): 125.76

Solvent: CHLOROFORM-d<smiles>COc1ccccc1CCC(=O)OC(C)(C)C</smiles>

$6 g$

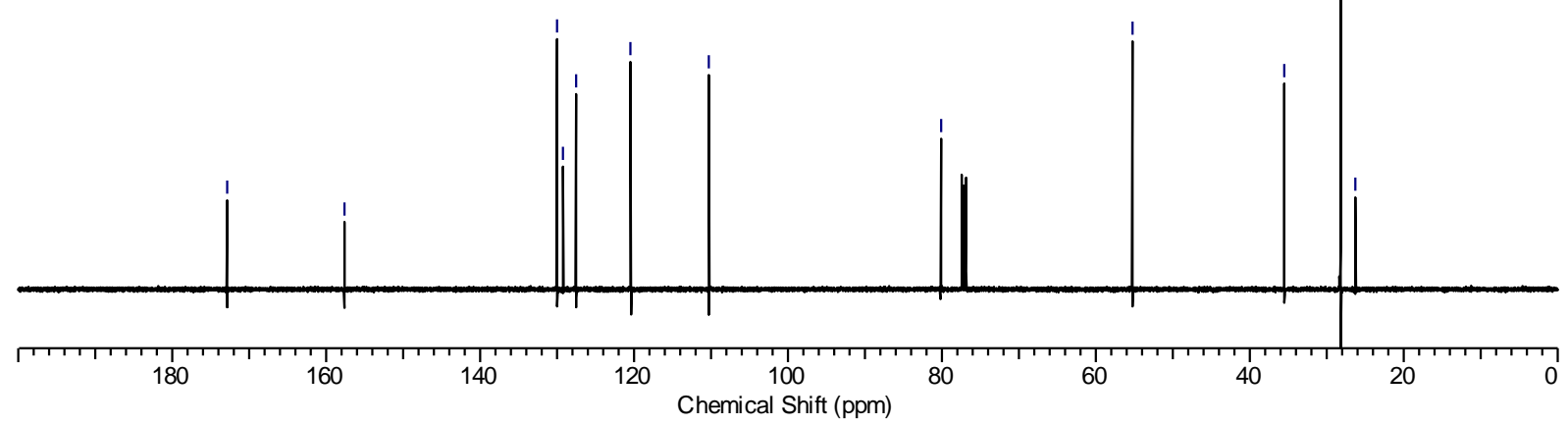


ND-590-5.010.esp

Frequency (MHz): 500.13

Solvent: CHLOROFORM-d

$\underbrace{O}_{6 h}$

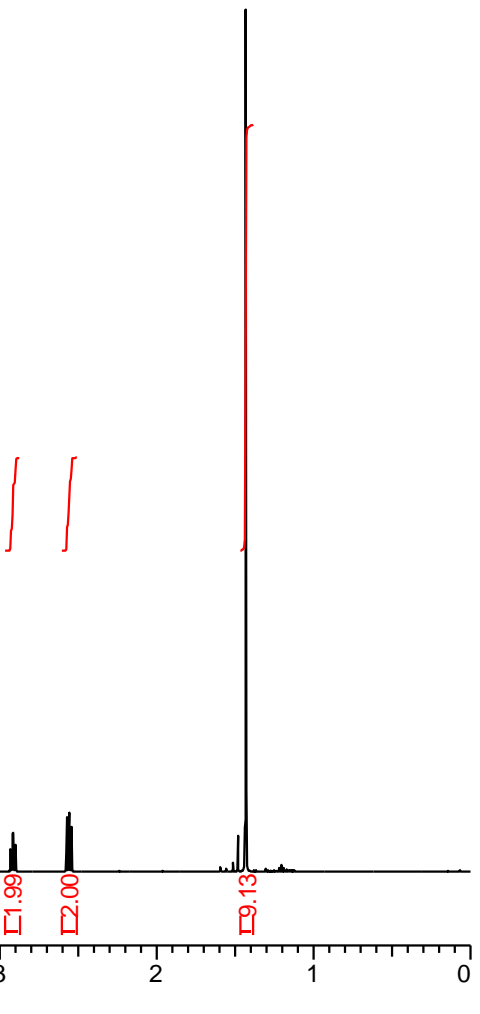

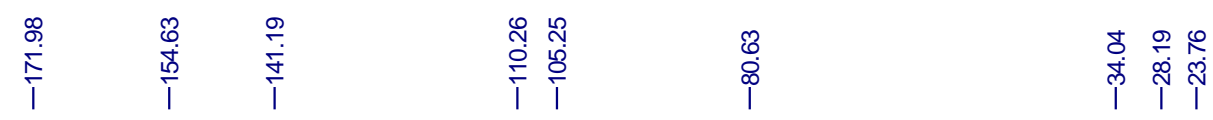

ND-590-5.011.esp

Frequency (MHz): 125.76

Solvent: CHLOROFORM-d

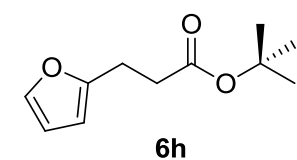

$6 h$

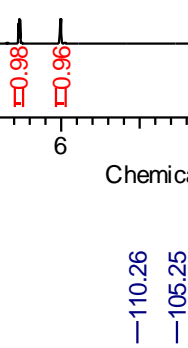

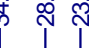

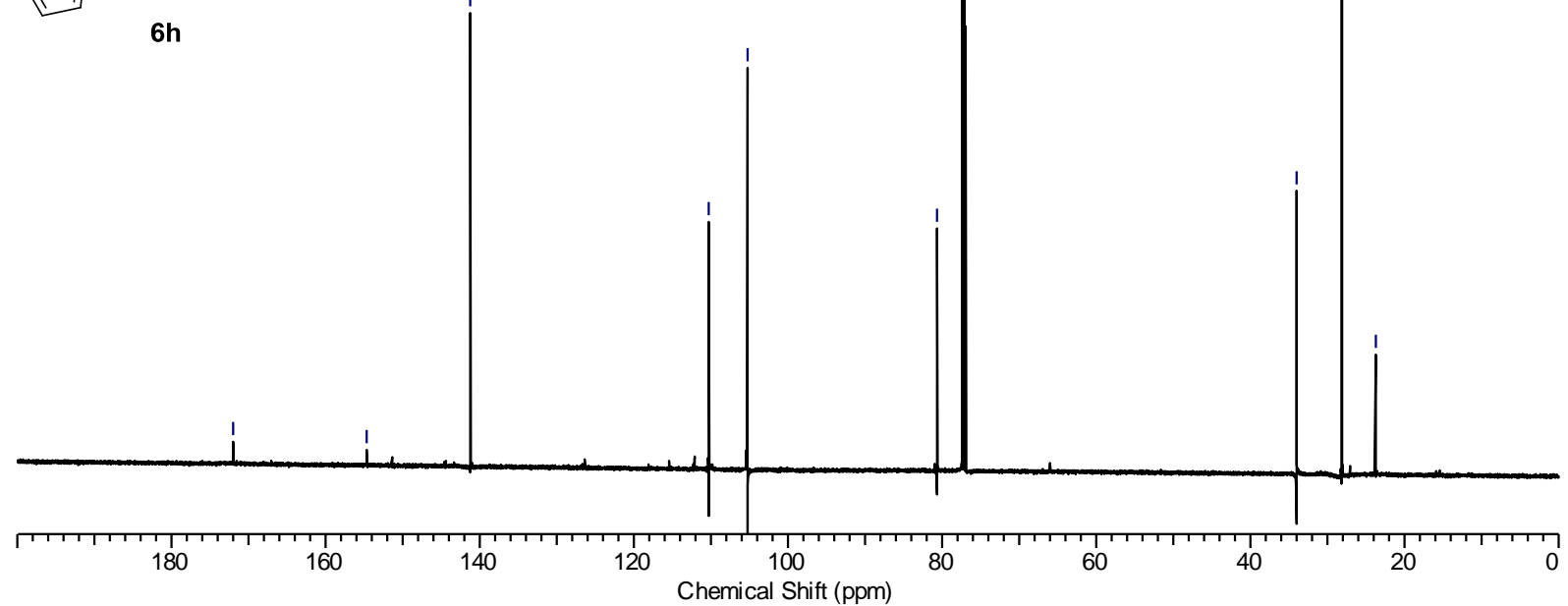


ND-590-2.010.esp

Frequency (MHz): 500.13

Solvent: CHLOROFORM-d<smiles>CC(C)(C)OC(=O)CCc1cccnc1</smiles>

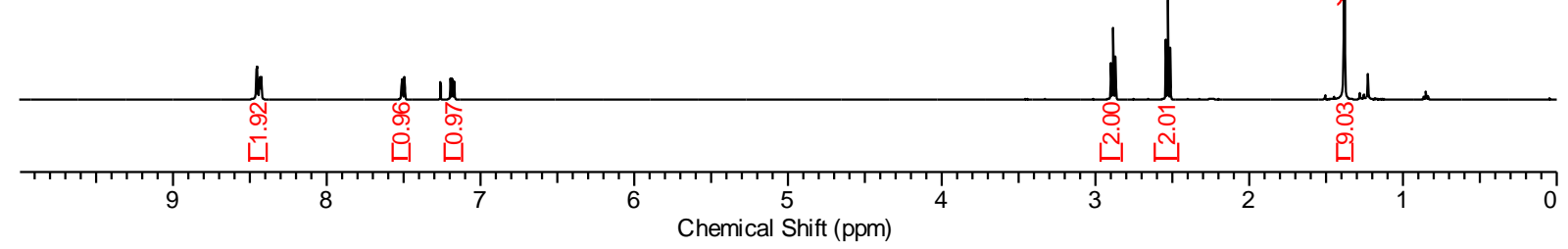

$$
\text { ए }
$$

$\infty$
$\infty$
$\infty$

ND-590-2.011.esp

Frequency (MHz): 125.76

Solvent: CHLOROFORM-d<smiles>CC(C)(C)OC(=O)CCc1cccnc1</smiles>

$6 \mathbf{i}$
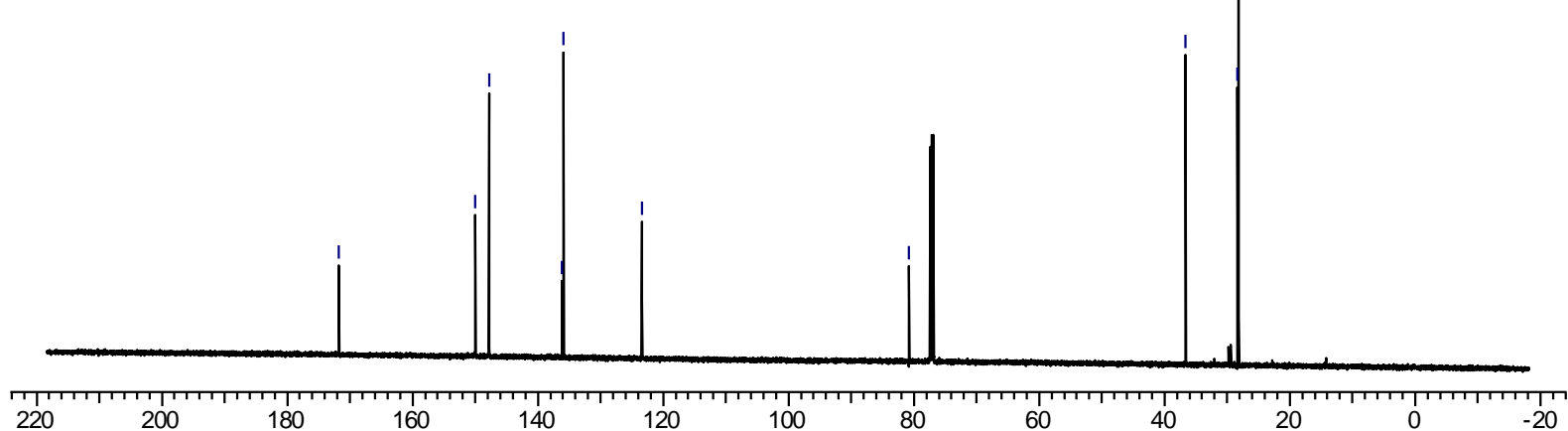
ND-588-1-SC_PROTON_20160523_01

Frequency (MHz): 299.86

Solvent: CHLOROFORM-d
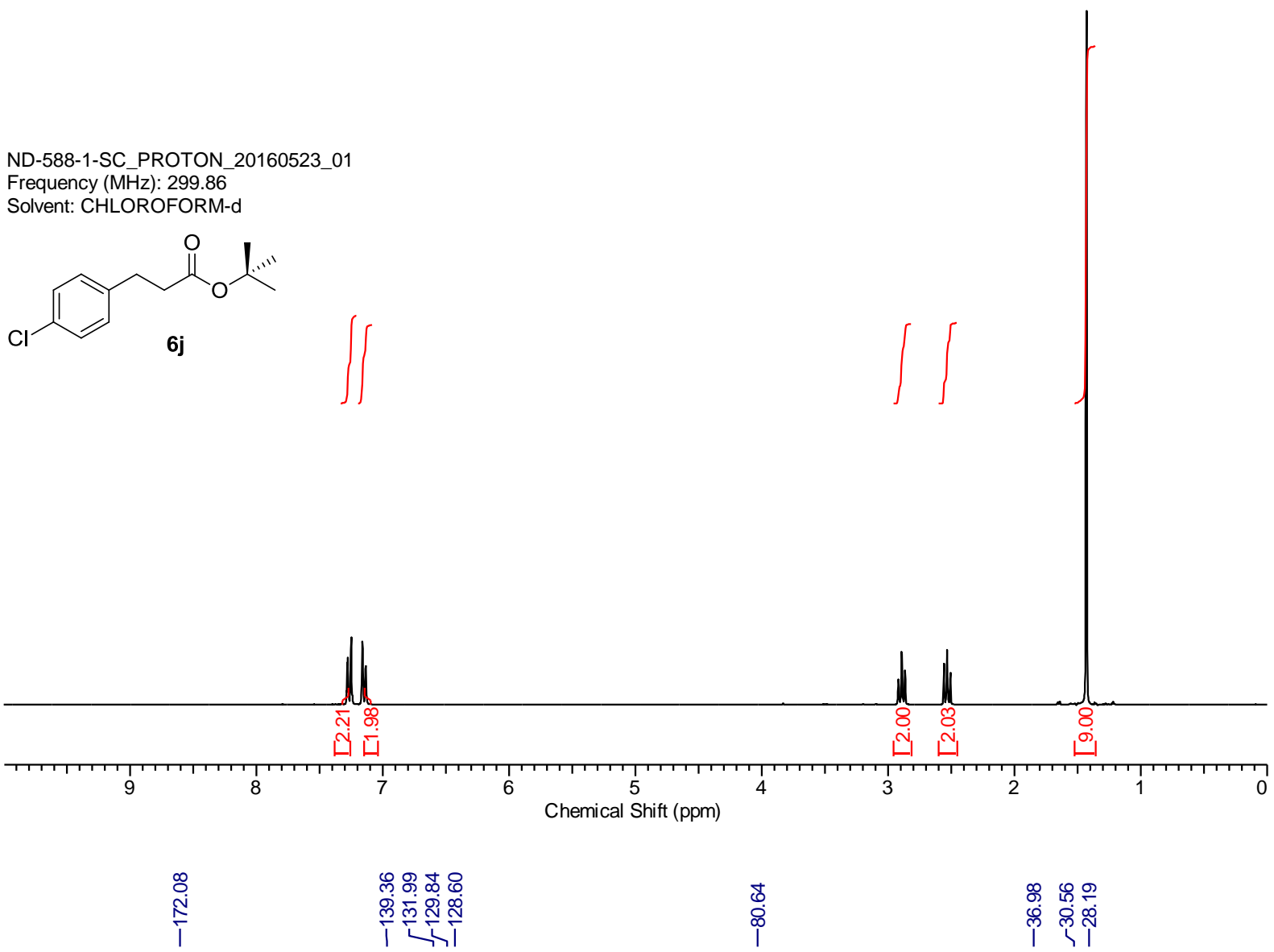

i

œ

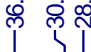

ND-528-4-F2.011.esp

Frequency (MHz): 125.76

Solvent: CHLOROFORM-d<smiles>CC(C)(C)OC(=O)CCc1ccc(Cl)cc1</smiles>

6j

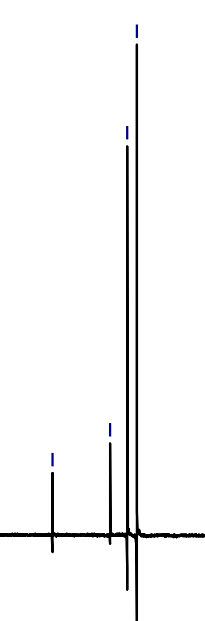

180

Chemical Shift (ppm)

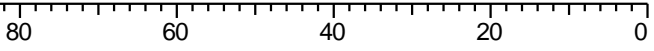


ND-527-7-SC.010.esp

Frequency (MHz): 500.13

Solvent: CHLOROFORM-d<smiles>CC(C)(C)OC(=O)CCc1ccc(F)cc1</smiles>

6k
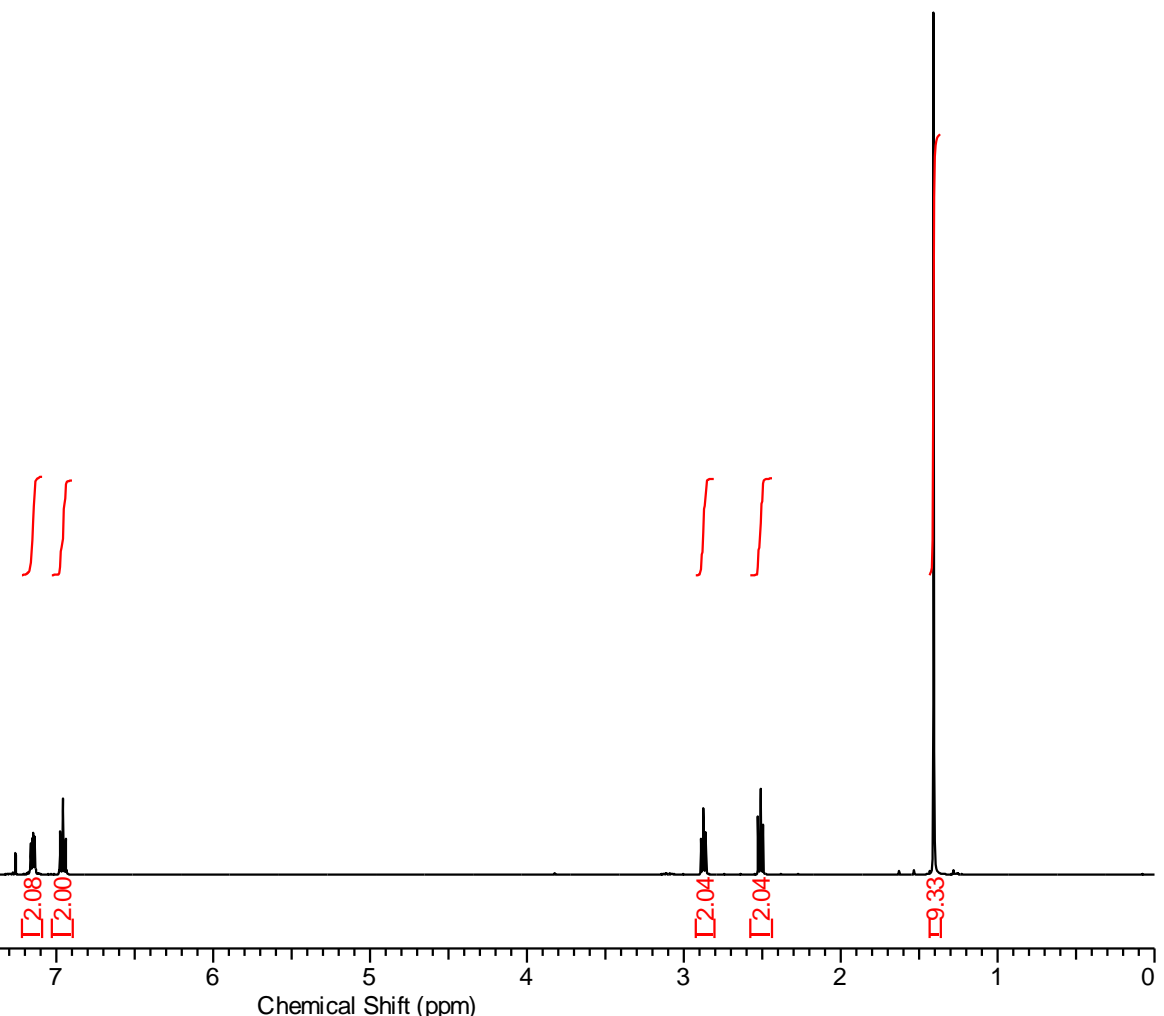

กำ ํํำ

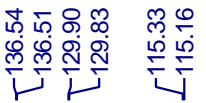

ณ̊

กิ ป⿻

ले 尺्र

ND-527-7-SC.011.esp

Frequency (MHz): 125.76

Solvent: CHLOROFORM-d<smiles>CC(C)(C)OC(=O)CCc1ccc(F)cc1</smiles>

6k

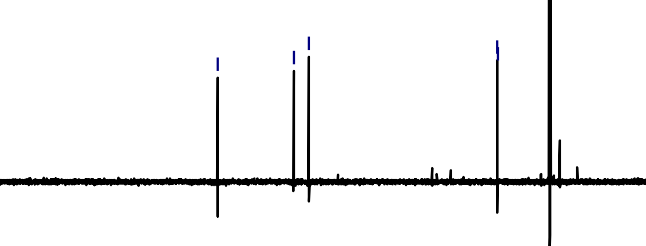

180

Chemical Shift (ppm)

80

(2) 

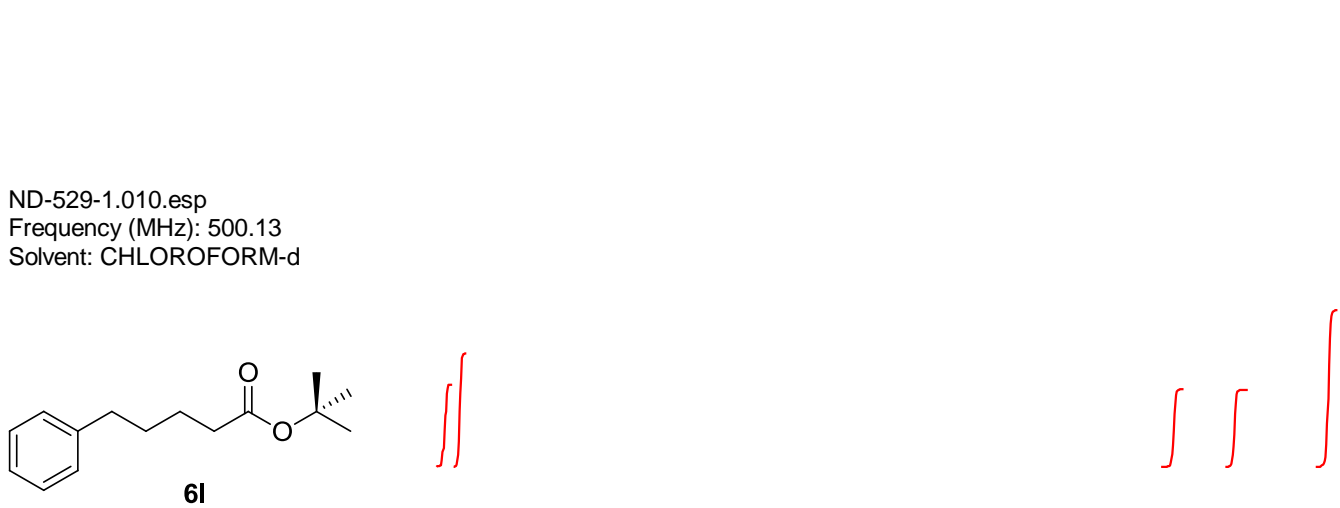

6I

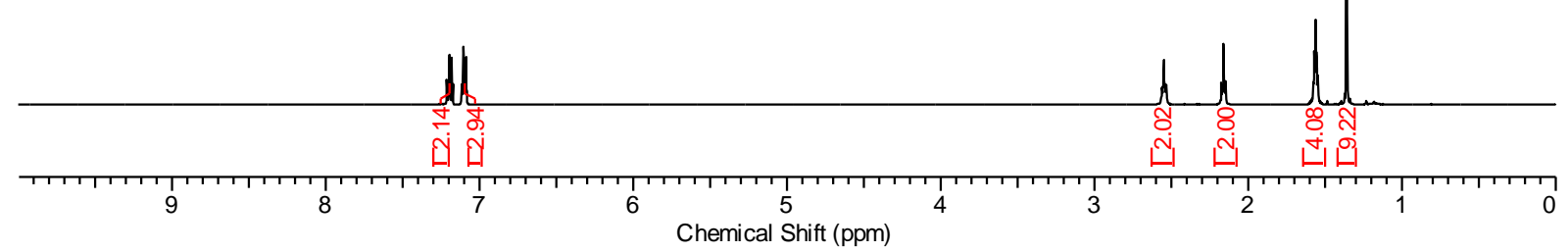

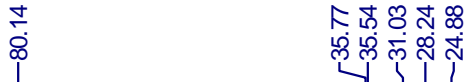

ND-529-1.011.esp

Frequency (MHz): 125.76

Solvent: CHLOROFORM-d<smiles>CC(C)(C)OC(=O)CCCCc1ccccc1</smiles>

61

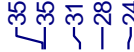

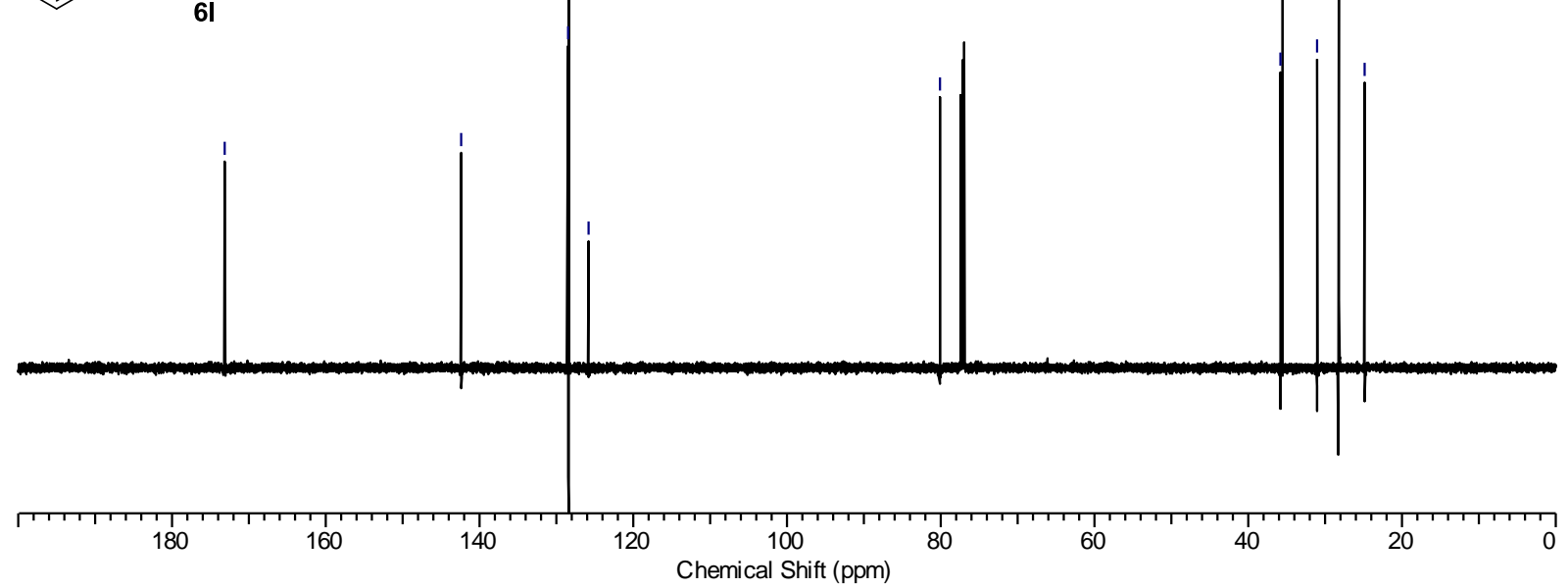


Follow-up products

ND-547-2-SC_PROTON_20160323_02 Frequency $(\mathrm{MHz}): 299.86$

Solvent: CHLOROFORM-d

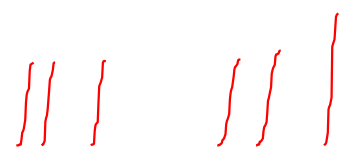<smiles>CCCCC(=O)CCc1ccc(OC)cc1</smiles>

$7 a$
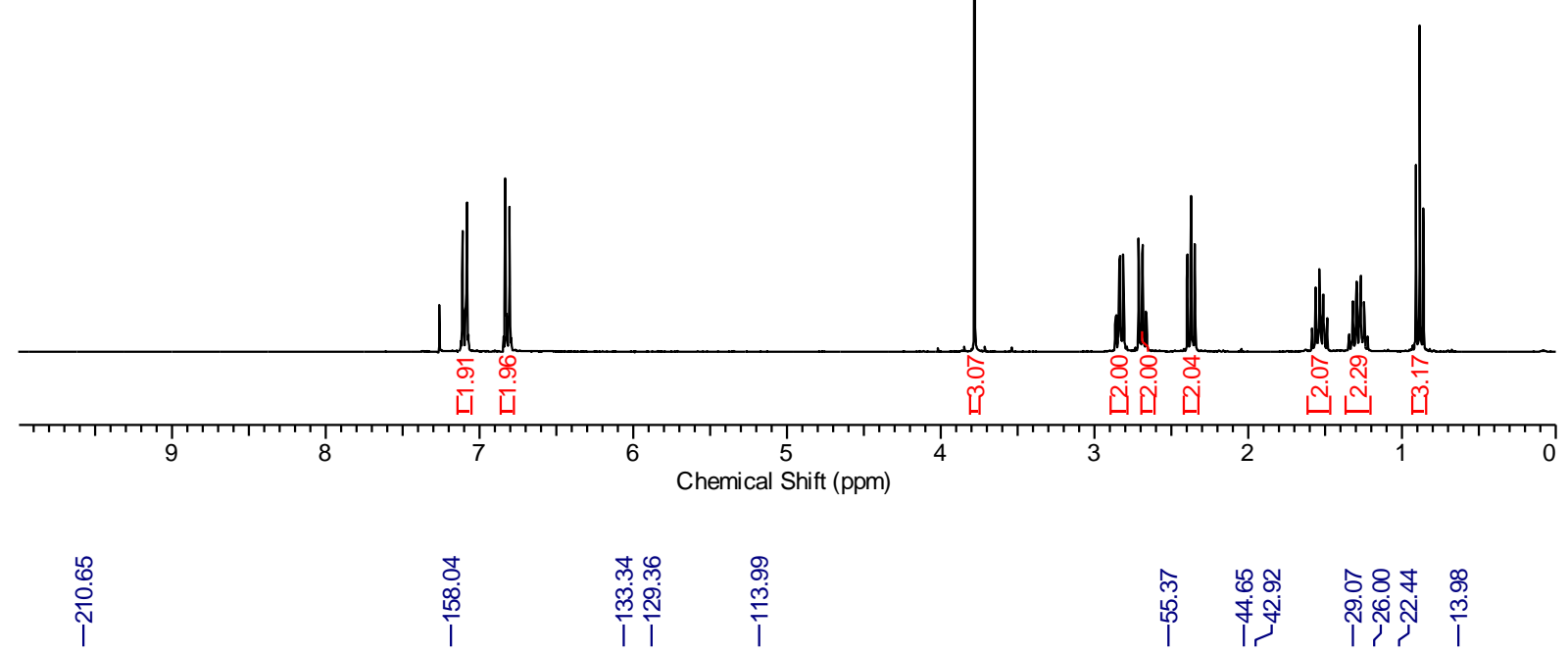

ND-547-2-SC CARBON 2016032301

Frequency (MHz): 75.41

Solvent: CHLOROFORM-d<smiles>CCCCC(=O)CCc1ccc(OC)cc1</smiles>

$7 a$

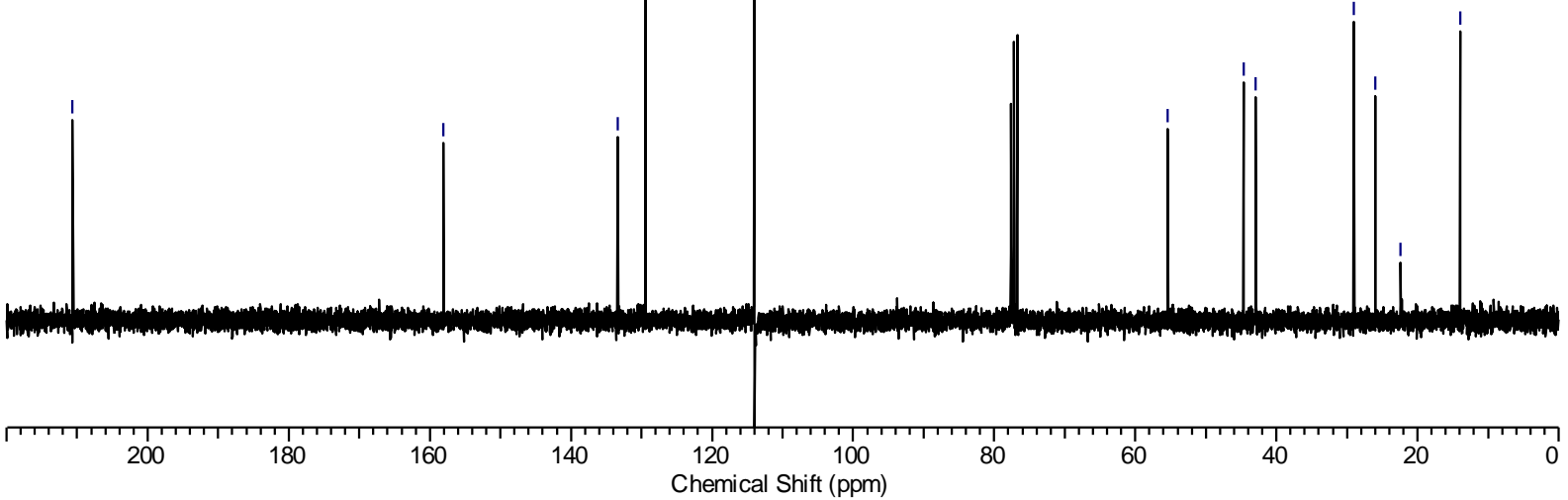


NSD-16-SC.010.esp

Frequency (MHz): 500.13

Solvent: CHLOROFORM-d

$\iiint$<smiles>COc1ccc(CCC(=O)c2ccc(C)cc2)cc1</smiles>

7b

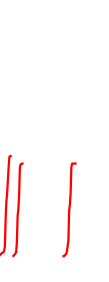

$\int$

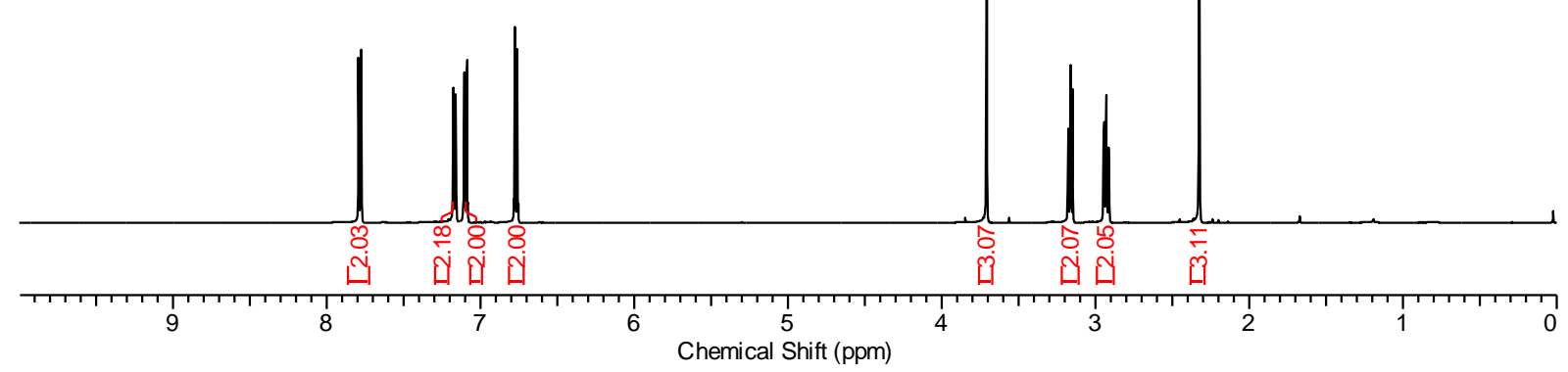
욤

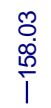

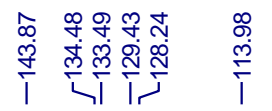

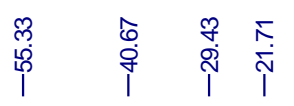

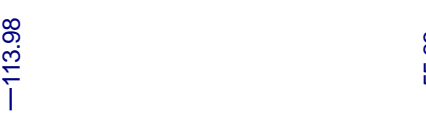

NSD-16-SC.011.esp

Frequency (MHz): 125.76

Solvent: CHLOROFORM-d<smiles>COc1ccc(CCC(=O)c2ccc(C)cc2)cc1</smiles> 
<smiles>COc1ccc(CCC(=O)c2ccccn2)cc1</smiles>

7c

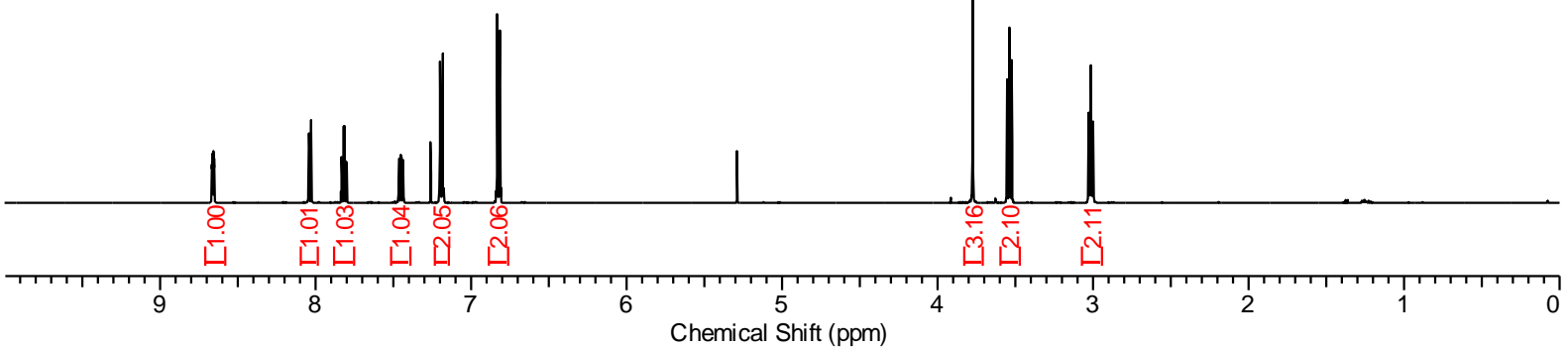

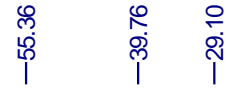

ND-577-2.011.esp

Frequency (MHz): 125.76

Solvent: CHLOROFORM-d<smiles>COc1ccc(CCC(=O)c2ccccn2)cc1</smiles>

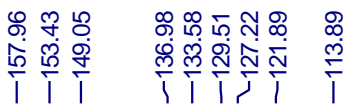

$\begin{array}{lll}m & 0 & 0 \\ \text { ñ } & \text { ल } & \text { N }\end{array}$

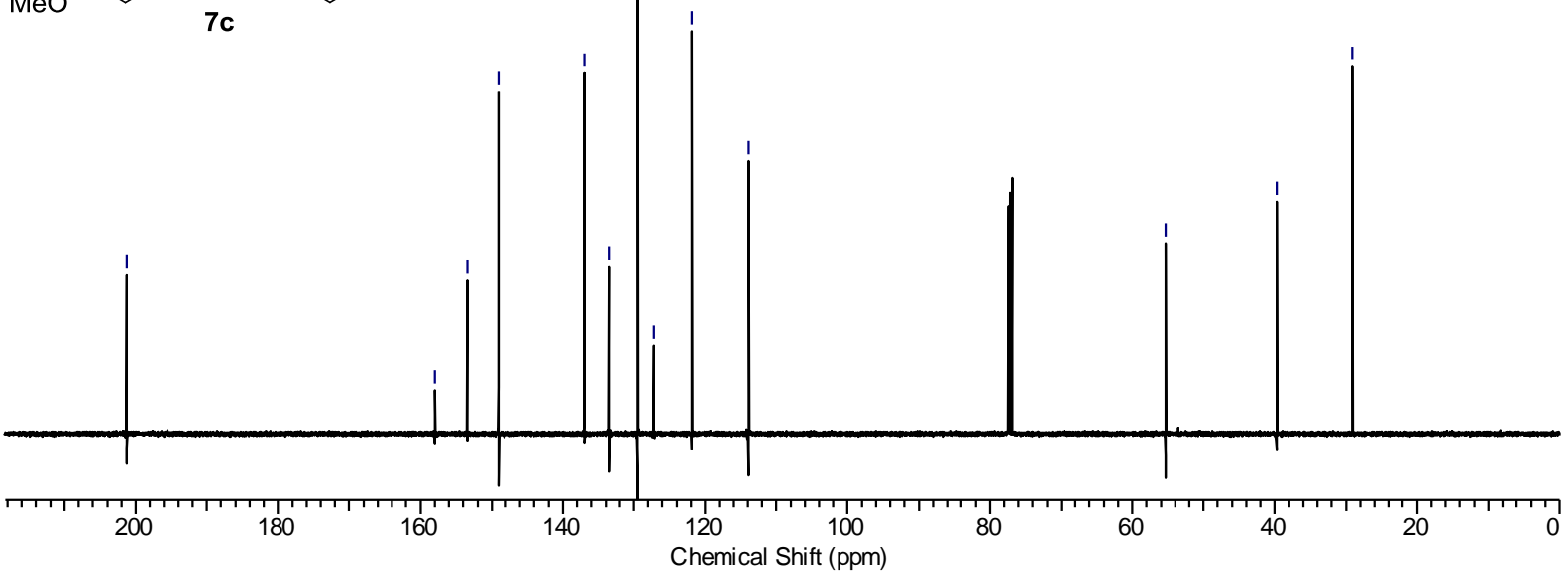


ND-601-F1.011.esp

Frequency $(\mathrm{MHz}): 500.13$

Solvent: CHLOROFORM-d
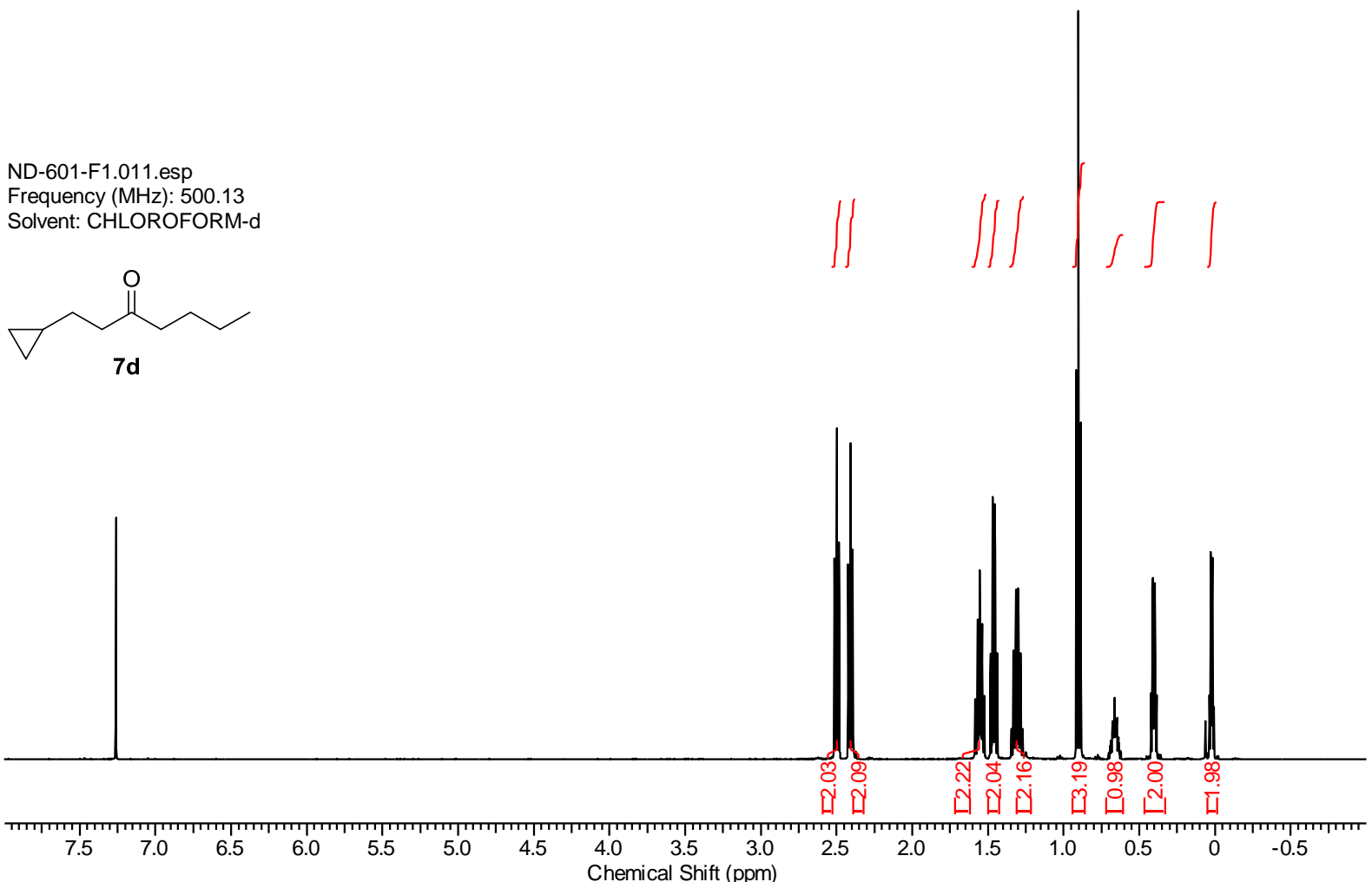

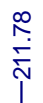

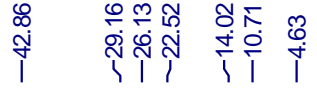

ND-601-F1.012.esp

Frequency (MHz): 125.76

Solvent: CHLOROFORM-d<smiles>CCCCC(=O)CCC1CC1</smiles>

7d

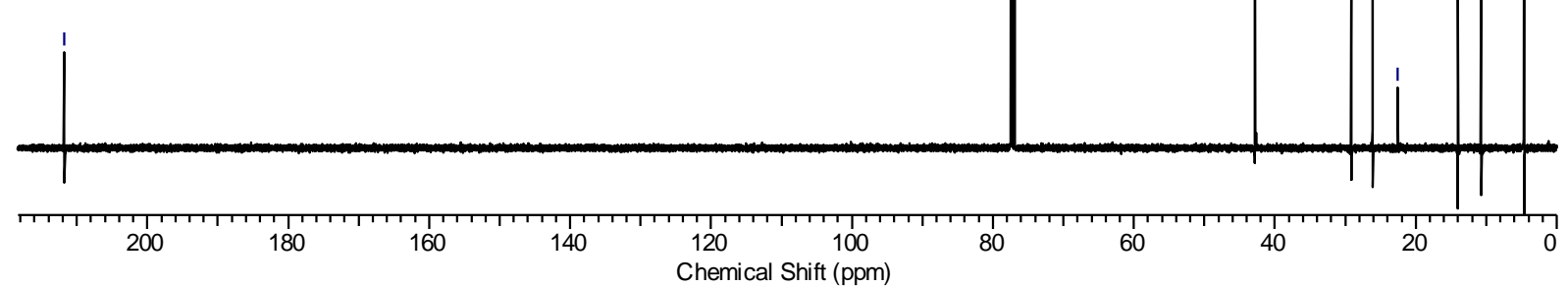


ND-548-SC_PROTON_20160309_02 Frequency ( $\overline{\mathrm{MHz}}$ ): $299 . \overline{8} 6$

Solvent: CHLOROFORM-d
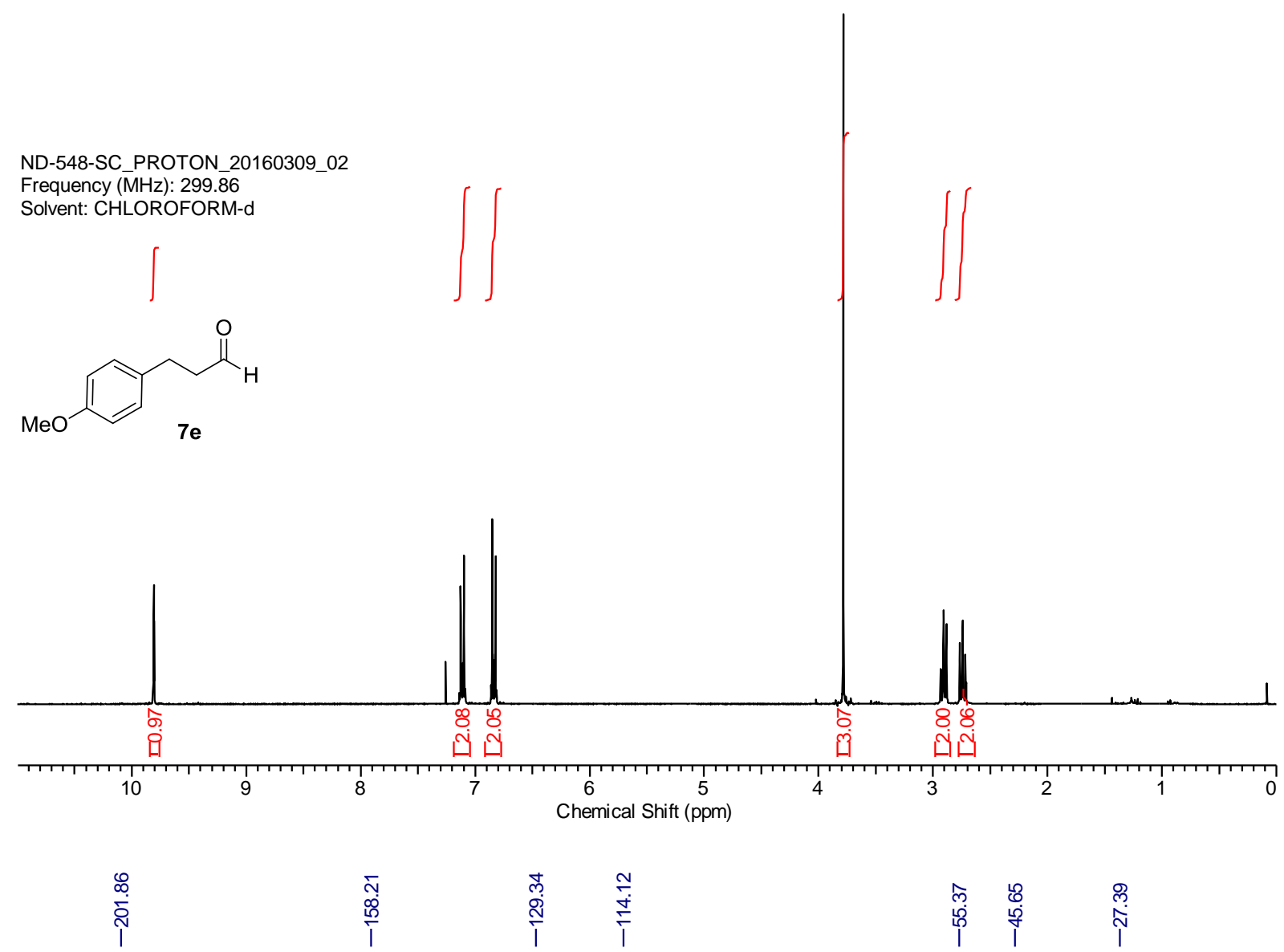

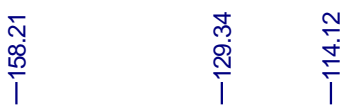

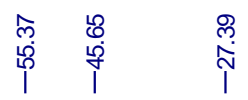

ND-548-SC_CARBON 2016030901

Frequency $(\mathrm{MHz})$ : 75.41

Solvent: CHLOROFORM-d

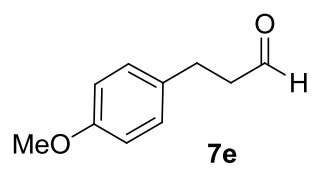

.

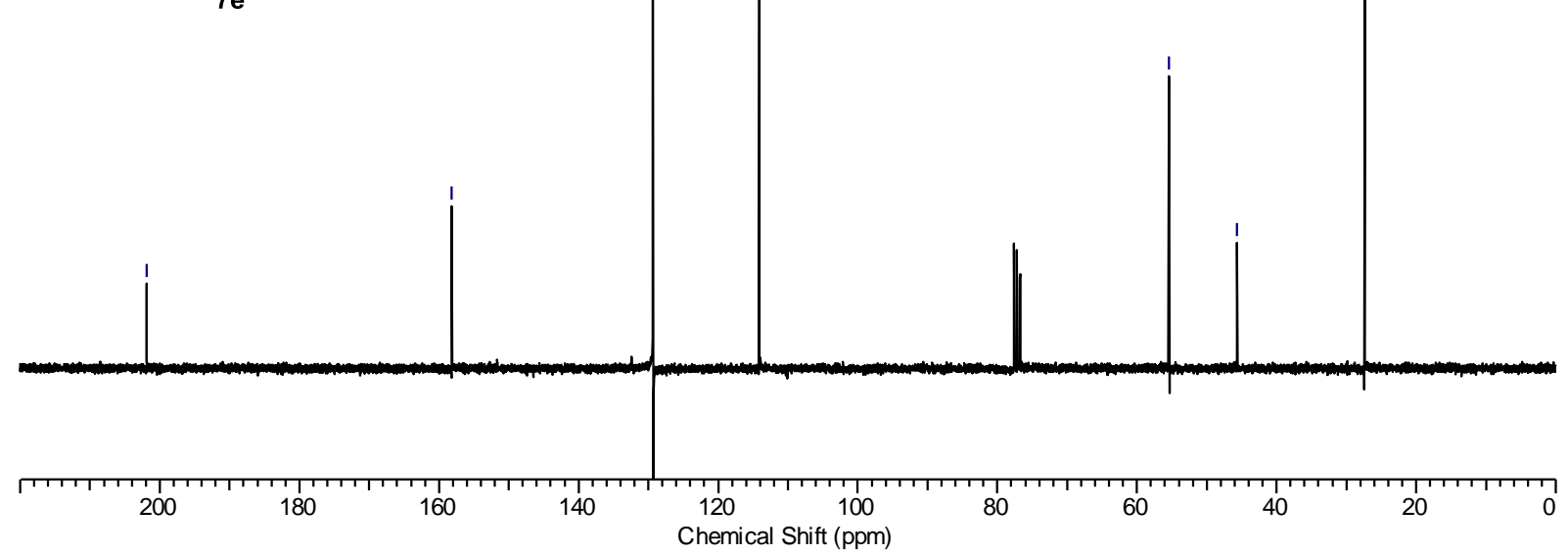


ND-600.020.esp

Frequency (MHz): 500.13

Solvent: CHLOROFORM-d

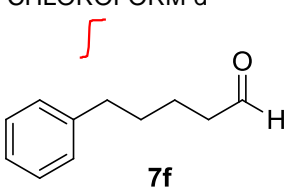

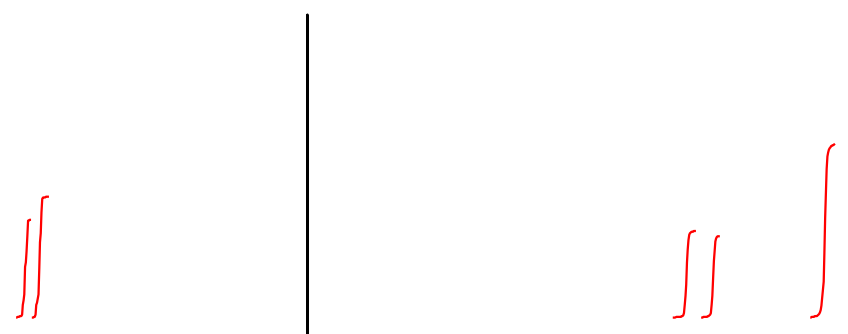

Dichloromethane (Reference)

वิㅇำ

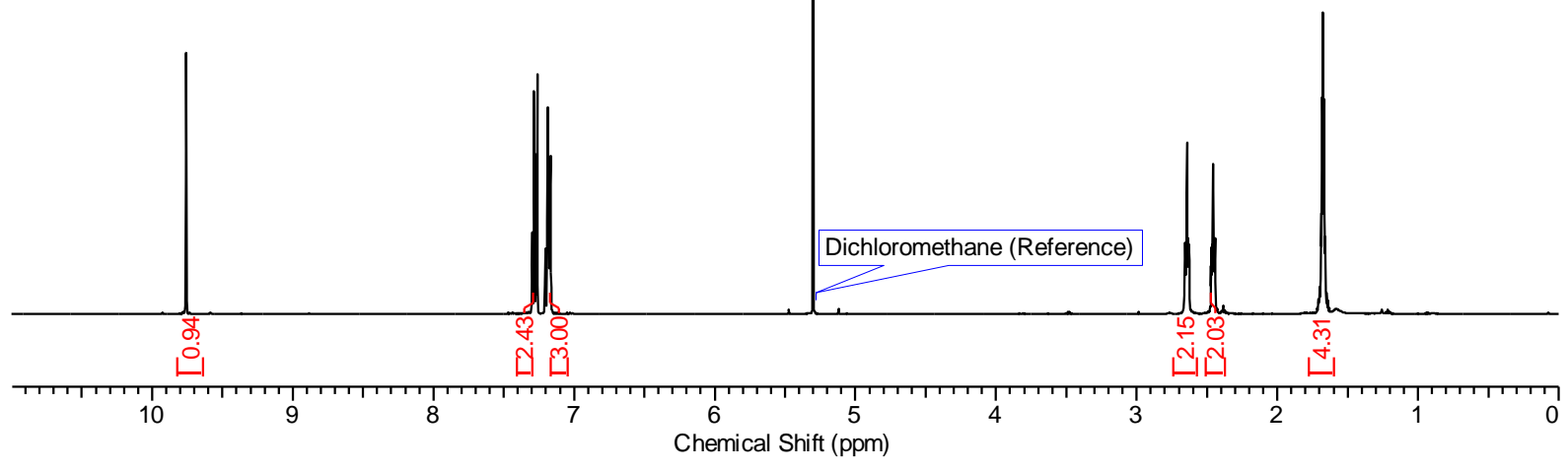

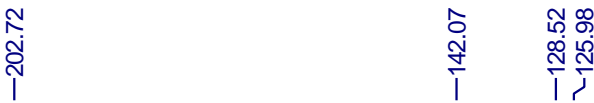

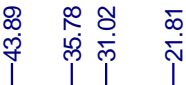

D-600.011.esp

Frequency (MHz): 125.76

Solvent: CHLOROFORM-d
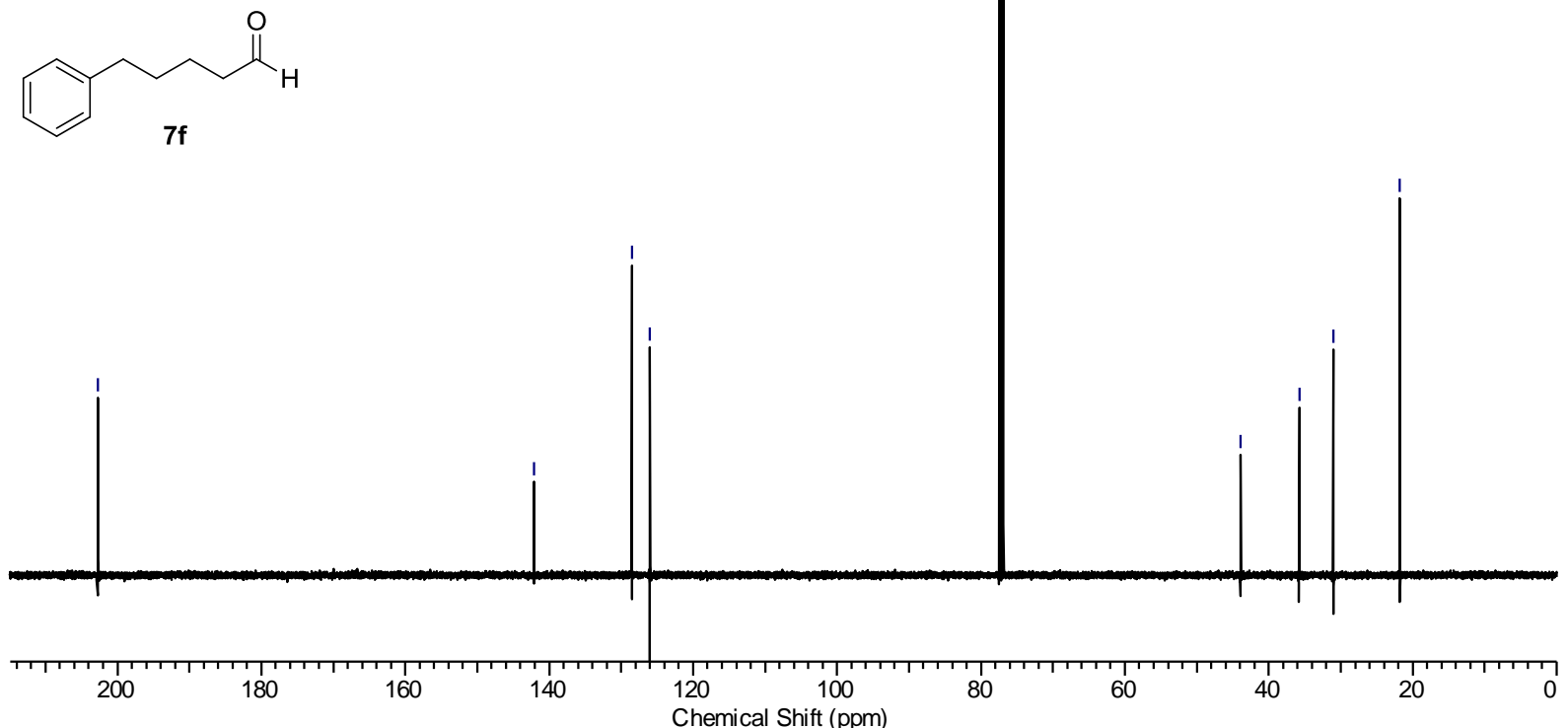


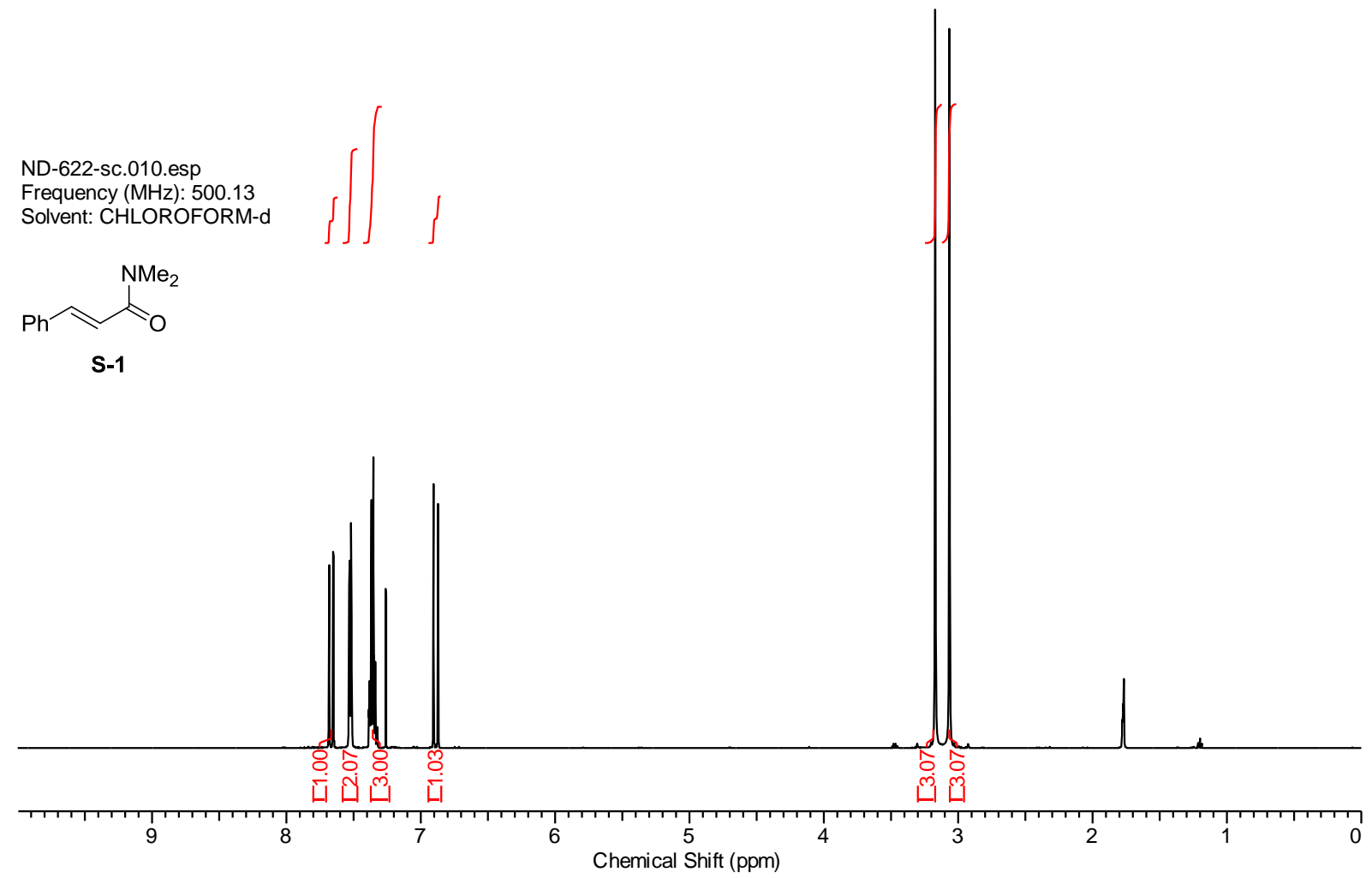

เก็ิ

लึं

\section{ND-622-sc.011.esp}

Frequency (MHz): 125.76

Solvent: CHLOROFORM-d<smiles>CN(C)C(=O)/C=C/c1ccccc1</smiles>

우 웅 망용

I ।

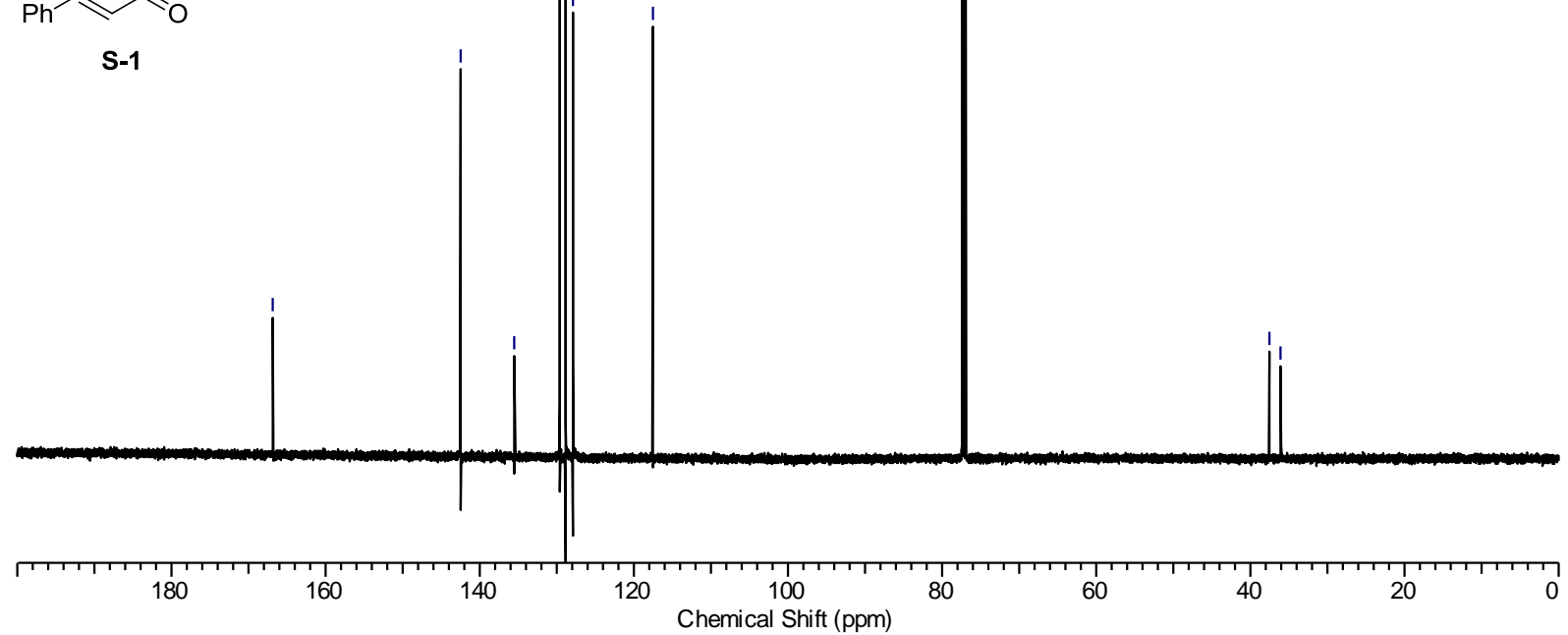




\section{References}

${ }^{1}$ (a) Michlik, S.; Kempe, R. Nat. Chem. 2013, 5, 140-144. (b) Michlik, S.; Kempe, R. Angew. Chem. Int. Ed. 2013, 52, 6326-6329.

2 (a) Rösler, S.; Ertl, M.; Irrgang, T.; Kempe, R. Angew. Chem. Int. Ed. 2015, 54, 15046-15050. (b) Rösler, S.; Obenauf, J.; Kempe, R. J. Am. Chem. Soc. 2015, 137, 7998-8001.

${ }^{3}$ Xu, K.; Hu, Y.; Zhang, S.; Zha, Z.; Wang, Z. Chem. Eur. J. 2012, 18, 9793-9797.

${ }^{4}$ Guo, L.; Liu, Y.; Yao, W.; Leng, X.; Huang, Z. Org. Lett. 2013, 15, 1144-1147.

${ }^{5}$ Molander, G. A.; Jean-Gerard, L. J. Org. Chem. 2009, 74, 5446-5450.

${ }^{6}$ Kuwahara, T.; Fukuyama, T.; Ryu, I. RSC Advances 2013, 3, 13702.

${ }^{7}$ Wu, Z.; Hull, K. L. Chem. Sci. 2016, 7, 969-975.

${ }^{8}$ Kokotos, C. G.; Baskakis, C.; Kokotos, G. J. Org. Chem. 2008, 73, 8623-8626.

${ }^{9}$ Guo, L.; Ma, X.; Fang, H.; Jia, X.; Huang, Z. Angew. Chem. Int. Ed. 2015, 54, 4023-4027.

${ }^{10}$ Iuchi, Y.; Obora, Y.; Ishii, Y. J. Am. Chem. Soc. 2010, 132, 2536-2537.

${ }^{11}$ Salome, C.; Kohn, H. Tetrahedron 2009, 65, 456-460.

${ }^{12}$ Xu, Q.; Chen, J.; Tian, H.; Yuan, X.; Li, S.; Zhou, C.; Liu, J. Angew. Chem. Int. Ed. 2014, 53, 225-229.

${ }^{13}$ Schaubach, S.; Gebauer, K.; Ungeheuer, F.; Hoffmeister, L.; Ilg, M. K.; Wirtz, C.; Furstner, A. Chem. Eur. J. 2016, 22, 8494-8507.

${ }^{14}$ Kelly, C. B.; Lambert, K. M.; Mercadante, M. A.; Ovian, J. M.; Bailey, W. F.; Leadbeater, N. E. Angew. Chem. Int. Ed. 2015, 54, 4241-4245. 